\title{
Are community-based health worker interventions an effective approach for early diagnosis of cancer?
}

DOI:

10.1002/pon.4575

\section{Document Version}

Accepted author manuscript

Link to publication record in Manchester Research Explorer

\section{Citation for published version (APA):}

Bellhouse, S., McWilliams, L., Firth, J., Yorke, J., \& French, D. (2017). Are community-based health worker interventions an effective approach for early diagnosis of cancer? A systematic review and meta-analysis. Psychooncology. https://doi.org/10.1002/pon.4575

\section{Published in:}

Psycho-oncology

\section{Citing this paper}

Please note that where the full-text provided on Manchester Research Explorer is the Author Accepted Manuscript or Proof version this may differ from the final Published version. If citing, it is advised that you check and use the publisher's definitive version.

\section{General rights}

Copyright and moral rights for the publications made accessible in the Research Explorer are retained by the authors and/or other copyright owners and it is a condition of accessing publications that users recognise and abide by the legal requirements associated with these rights.

\section{Takedown policy}

If you believe that this document breaches copyright please refer to the University of Manchester's Takedown Procedures [http://man.ac.uk/04Y6Bo] or contact uml.scholarlycommunications@manchester.ac.uk providing relevant details, so we can investigate your claim.

\section{OPEN ACCESS}




\section{Psycho-Oncology}

\section{Are community-based lay health worker interventions an effective approach for early diagnosis of cancer? A systematic review and meta-analysis}

\begin{tabular}{|r|l|}
\hline Journal: & Psycho-Oncology \\
\hline Manuscript ID & PON-17-0368.R1 \\
\hline Wiley - Manuscript type: & Review Article \\
\hline Complete List of Authors: & $\begin{array}{l}\text { Bellhouse, Sarah; The Christie NHS Foundation Trust, Christie Patient } \\
\text { Centred Research, School of Oncology } \\
\text { McWilliams, Lorna; The Christie NHS Foundation Trust, Christie Patient } \\
\text { Centred Research, School of Oncology } \\
\text { Firth, Joseph; The University of Manchester, Division of Psychology and } \\
\text { Mental Health, School of Health Sciences; Western Sydney University } \\
\text { School of Science and Health, NICM } \\
\text { Yorke, Janelle; University of Manchester, Division of Nursing, Midwifery and } \\
\text { Social Work, School of Health Sciences; The Christie NHS Foundation Trust, } \\
\text { Christie Patient Centred Research, School of Oncology } \\
\text { French, David; The University of Manchester, Manchester Centre for Health } \\
\text { Psychology, School of Health Sciences }\end{array}$ \\
\hline Keywords: & $\begin{array}{l}\text { cancer, oncology, behavior change techniques, early diagnosis, meta- } \\
\text { analysis, systematic review, community health worker }\end{array}$ \\
\hline
\end{tabular}

\section{SCHOLARONE"}

Manuscripts 
1

2

3

4

5

6

7 Running title: CBHWs and Early Diagnosis of Cancer

8 Authors' affiliations:

$9{ }^{1}$ Christie Patient Centred Research Group, School of Oncology, The Christie NHS Foundation 10 Trust, UK

$11{ }^{2}$ Division of Psychology and Mental Health, School of Health Sciences, University of

12 Manchester, UK

${ }^{3}$ NICM, School of Science and Health, University of Western Sydney, Australia

${ }^{4}$ Division of Nursing, Midwifery and Social Work, School of Health Sciences, University of Manchester UK

${ }^{5}$ Manchester Centre for Health Psychology, School of Health Sciences, University of Manchester, UK

\section{Corresponding author:}

Sarah Bellhouse, Christie Patient Centred Research Group, School of Oncology, The Christie NHS Foundation Trust, Wilmslow Road, Withington, Manchester, M20 4BX, UK. Phone: 0161 918 2451; E-mail: sarah.bellhouse@christie.nhs.uk

\section{Word count: 6,259}

Figures/tables: 4 


\section{Abstract}

2 Objective: This systematic review aimed to assess the effectiveness of community-based health

3 worker (CBHW) interventions for early detection of cancer. Secondary aims were to consider the

4 extent that interventions were based on theory, and potential moderators including behaviour

5 change techniques (BCTs).

6 Methods: Six databases were searched for randomized controlled trials. Random-effects meta-

7 analyses were applied to 30 eligible studies with a cancer screening outcome.

8 Results: Participation in CBHW interventions was associated with increased receipt of screening

$9(\mathrm{OR}=1.901,95 \% \mathrm{CI}: 1.60-2.26, \mathrm{p}<0.001)$ for breast, cervical and bowel cancer. Larger effect

10 sizes were observed in participants previously non-adherent with recommended schedules of

11 cancer screening. 25/30 studies were conducted with ethnic minority groups. Only 15 (45\%)

12 studies explicitly reported a theoretical foundation for intervention. The number of BCTs used by

13 CBHWs had a trend level association with observed effect size $(p=0.08)$. Study quality was

14 generally poor and common limitations were inadequate blinding and reliance on self-reported 15 outcomes.

16 Conclusions: CBHW interventions are an effective resource for increasing uptake of all three

17 types of cancer screening in ethnic minority groups. Those previously non-adherent with

18 recommended schedules of cancer screening benefitted the most from the CBHW approach.

19 However, better quality studies based on more explicit evidence-based theory are needed to

20 optimise the effectiveness of CBHW interventions on screening uptake. Further research is 
1

2

3

4

5

6

7

8

9

10

11

12

13

14

15

16

17

18

19

20

21

22

23

24

25

26

27

28

29

30

31

32

33

34

35

36

37

38

39

40

41

42

43

44

45

46

47

48

49

50

51

52

53

54

55

56

57

58

59

60

1 needed to ascertain whether CBHWs can help promote symptom recognition and help-seeking

2 behaviour to facilitate early diagnosis of cancer.

3 Keywords: behaviour change techniques, cancer, early diagnosis, community health worker,

4 meta-analysis, oncology and systematic review

5

6

7

8

9

10

11

12

13

14

15

16

17

18 


\section{1. Background}

2 In 2013, The World Health Organization (WHO) published their global action plan for the

3 prevention and control of non-communicable diseases, including cancer. ${ }^{1}$ The WHO recognize

4 that not all cancers are preventable and so health services are urged to focus on diagnosing

5 cancer earlier in order to increase survival rates and reduce costs associated with treating the

6 disease. $^{2}$ To facilitate this, more interventions aimed at 'down-staging' cancers such as

7 increasing uptake of screening programmes are warranted. ${ }^{3}$

8 Even though recognition of cancer signs and symptoms are relatively high ${ }^{4}$, attendance rates for

9 cancer screening have decreased in recent years in the UK and USA. ${ }^{5-7}$ Barriers to attending

10 screening or raising a cancer-related health concern with healthcare professionals remain

11 including; language/cultural barriers, embarrassment/fear and perceptions of wasting doctors'

12 time. $^{8-10}$

13 One approach to overcoming barriers towards cancer screening and early diagnosis is using Lay

14 Health Workers (LHWs) i.e., any health worker carrying out functions related to health care

15 delivery who does not have any formal professional of paraprofessional qualifications. ${ }^{11,12}$

16 LHW roles in a cancer context include providing education to raise cancer awareness and

17 overcoming individual barriers to attending screening. Two LHW models have been proposed in

18 the literature, differentiated by setting; community based and clinic/hospital based. ${ }^{13}$ Although

19 there is overlap in tasks completed by both types of LHW, community-based health workers

20 (CBHWs) act as a liaison facilitating initial access between individuals and healthcare settings.

$21{ }^{12,14}$ In contrast, LHWs based in clinic and hospital settings, commonly referred to as patient

22 navigators, offer guidance to persons with an abnormal cancer screening test or a cancer 
1 diagnosis to access the cancer care system. ${ }^{15,16}$ The present review will focus on CBHWs only as

2 they provide the earliest possible opportunity to promote early cancer diagnosis.

3

4 Several systematic reviews have examined the effects of CBHW interventions on cancer

5 outcomes, most commonly cancer screening uptake. ${ }^{13,17-21}$ Two previous systematic reviews

6 which applied meta-analytic techniques found that CBHWs can improve breast cancer screening

7 uptake, with small effects of $1.05^{11}$ and $1.06^{22}$ reported.

8 Although these previous systematic reviews are useful, they have four limitations that the present

9 research aims to address. Firstly, the searches in the previous systematic reviews were conducted

10 some time ago (2001 and 2008), so there is a need to update systematic review evidence in this

11 area. Secondly, previous reviews that have included quantitative estimates of effects on

12 screening attendance have only examined mammography uptake, one of several available

13 screening programmes in many countries. Since then, more recent studies have examined the

14 effects of CBHWs on other forms of screening ${ }^{23-25}$, potentially allowing quantitative estimates of

15 the effects of CBHW interventions on these other kinds of screening. Thirdly, previous reviews

16 have not considered the use of theory underpinning intervention development on effectiveness

17 beyond listing theories mentioned. Consequently, it is difficult to assess the extent to which

18 these theories are used in the design, analysis and interpretation of review results. Fourth,

19 previous reviews have not assessed how the use of behaviour change techniques (BCTs) may

20 enhance early detection outcomes. BCTs have been defined as "observable, replicable, and

21 irreducible component(s) of an intervention designed to alter or redirect causal processes that

22 regulate behaviour" $(\mathrm{p} 82)^{26}$, and have been shown to moderate the effects of interventions for 
1 other behaviours. ${ }^{27}$ Thus, previous reviews have not clearly described the active intervention

2 ingredients that CBHW interventions include.

3 The aim of the present review was to systematically identify and critically review studies that

4 investigate the effectiveness of CBHWs in promoting outcomes pertinent to early cancer

5 diagnosis including screening, symptom recognition, early detection, and help-seeking

6 behaviour. Specific objectives were to:

7 a) determine the effectiveness of lay health led interventions in targeting outcomes related

8 to early detection of cancer, with the expectation that screening uptake will be the likely

9 focus considering previous reviews in this area.

10 b) identify the extent to which risk of bias may affect intervention effectiveness.

11 c) assess the extent to which CBHW interventions are underpinned by a theoretical

12 foundation and determine the prevalence and types of BCTs included in interventions.

13 d) examine potential moderators of intervention effectiveness, including type of screening 14 programme, mode of delivery, intervention setting, and use of BCTs.

\section{Methods}

\section{2.1. Search strategy}

18 This systematic review followed the PRISMA statement, with no ethical approval required. ${ }^{28}$

19 Electronic database searches were conducted of MEDLINE, Embase, BNI, CINAHL, PsycINFO

20 \& AMED in May 2016, and updated in April 2017. No date restrictions were applied for any 
1 searches. A broad search strategy was used to identify relevant articles and variation of index

2 terms across databases was taken into account. For the full search strategy for each database, 3 refer to Appendix S1.

4

5 In addition to database searches, forward and backward citation searches were conducted.

6 Companion papers were retrieved where necessary to ascertain further information about

7 intervention development and content and CBHW selection/training.

8

9 2.2. Eligibility criteria

10 Only English-language research articles published in peer-reviewed journals were included in the 11 review. Studies were included if they met the following criteria:

12 (1) Participants: general population ( $\geq 18$ years).

13 (2) Interventions were delivered by a CBHW who did not require formal professional or 14 paraprofessional education/qualifications to complete the role. The role of the CBHW 15 was to facilitate initial engagement with healthcare providers and access to services related to early cancer diagnosis e.g. cancer screening or visiting the GP after identifying a potential cancer symptom. Therefore studies delivered in primary care settings would be eligible if the person was attending for a routine visit unrelated to cancer. Studies whereby CBHWs were providing follow up support for those already engaged with cancer related healthcare were excluded. Studies were excluded if CBHW input could not be assessed where healthcare professionals were also involved. Interventions were included whether the CBHW did or did not receive payment for their work. 
(3) Controls: studies had to have at least one non CBHW comparator arm, with no other restriction placed upon the nature of the comparison with the intervention group.

(4) Outcomes: studies had to measure an outcome that promoted early diagnosis of cancer at baseline and post-intervention e.g. uptake/intention to complete cancer screening programmes or any measure of cancer symptom awareness/beliefs, knowledge of cancer risk factors, benefits of early detection and help seeking behaviour/confidence to detect a change that might be cancer. There had to be an explicit link between the targeted outcome and cancer.

(5) Studies: RCTs and cluster RCTs were included. Only randomized trials with at least one comparator arm were included in this review because these study designs provide the strongest evidence for evaluating effectiveness of interventions. ${ }^{29}$

\subsection{Study selection}

All studies identified during the database search were assessed for relevance in a two-stage screening process; firstly the studies were assessed based on the information contained in the title and abstract. If at this stage the study appeared to meet the inclusion criteria, or if this was unclear, the full article was retrieved. Papers selected for retrieval were primarily assessed by

17 one reviewer (SB) to determine inclusion given the clearly defined a-priori criteria. In ambiguous cases, additional reviewers were consulted (LM, JY, DF).

\subsection{Data extraction}

Detailed information on sample demographics, CBHW characteristics (including selection/training processes) and intervention characteristics was extracted and an example 
1 extraction form is available in Appendix S2. Relevant information was also extracted to code

2 theory use, assess BCT prevalence and judge risk of bias, coded by one reviewer (SB). Five

3 articles were double coded by two reviewers (SB, LM) to establish agreement. During the double

4 coding process, advice was sought from a third reviewer (DF) regarding definitions of

5 terminology to ensure consistency and fairness in coding. Substantial agreement was indicated

6 for theory coding (chance-corrected $\kappa=.72$ ) and risk of bias judgements (chance-corrected $\kappa=.90$ )

7 and moderate agreement for BCTs (chance-corrected $\kappa=.0 .55)$. However it is not recommended

8 to use the Kappa statistic when coding the BCT taxonomy due to the high likelihood of BCTs

9 being absent artificially lowering observed Kappa statistics. ${ }^{30}$ Given this, reporting percentage

10 agreement is more appropriate; this was almost perfect at $97 \%$. Due to high agreement, one

11 reviewer (SB) proceeded to code the remaining 30 articles and advice was sought from a third

12 reviewer (DF) on an adhoc basis.

Theory application. The Theory Coding Scheme ${ }^{31}$, a coding framework developed to

14 assess extent of behaviour change theory use was used to code reported theory application in 15 included articles. The framework consists of 33 questions which are rated "yes", "no", "don’t

16 know" or "n/a". It has previously been used in systematic reviews to code theory use based on 17 intervention reports. ${ }^{32}$ The Theory Coding Scheme assesses the extent to which intervention 18 techniques are explicitly linked to theoretical constructs. A theoretical construct is defined as a 19 concept within a theory which predicts behaviour and is amenable to change e.g. perceived 20 susceptibility in the Health Belief Model. ${ }^{33}$ Percentages were calculated to assess the number of 21 studies scoring "yes" to each question. An overall theory score (range 0-8) was calculated using 22 the answers to items 3 to $11 .^{32}$ 
Behaviour change technique use. A hierarchical taxonomy of 93 BCTs was used to

2 code the content of the interventions. ${ }^{26}$ The most recent hierarchical list was used, with published

3 definitions guiding coding for each technique. The lead coder (SB) completed the online training

4 resource for the BCT taxonomy. A BCT was not coded as present unless the text explicitly

5 linked the technique to both the named target behaviour and the named population. For example,

$6 \quad$ "CBHWs helped participants identify their barriers to cancer screening and generated possible

7 solutions to help overcome the barriers" adequately describes the problem solving BCT whereas

8 stating that "barriers to cancer screening were discussed" is insufficient. Percentages were

9 calculated to assess the proportion of studies using each BCT.

10 Risk of bias. Using the Cochrane Collaboration's tool for assessing risk of bias ${ }^{34}$, a

11 judgement of risk of bias was made for items related to randomization and blinding procedures,

12 incomplete data assessment, selective reporting and any other sources of bias. The additional

13 guidance provided by the Cochrane Collaboration was followed when deciding between low, 14 unclear or high risk judgements.

\subsection{Statistical analyses}

17 Meta-analyses of screening attendance measures were performed in Comprehensive Meta-

18 Analysis $2.0 .^{35}$ To account for the expected heterogeneity between studies, a random-effects

19 model was applied. ${ }^{36}$ First, 'adherence to cancer screening' was calculated as a pooled odds ratio

20 (O.R.) comparing the total numbers of participants who had taken part in screening for breast

21 cancer, colorectal cancer or cervical cancer following CBHW interventions versus control 
1 conditions. Breast self-examination (BSE) and/or clinical breast examination (CBE) adherence

2 data were not classified as forms of cancer screening in these analyses. Data from both medical

3 records and self-report measures were used for analysis. Where studies had reported this

4 separately, a mean O.R. was calculated for medical record and self-report data and used in the

5 analysis. Similarly, where studies which had reported multiple cancer screening outcomes, a

6 mean O.R. was calculated from all eligible measures within each respective study.

7 The secondary outcome was 'Uptake of cancer screening amongst non-adherent samples'. 'Non-

8 adherent" refers to individuals, who at the time of participation in the study were non-compliant

9 with age appropriate recommended schedules of cancer screening. Thus "uptake" refers to the

10 total number of non-adherent individuals who attended cancer screening after receiving a CBHW

11 intervention (or control condition). Data from those studies which included only non-adherent

12 samples from the outset, and those studies which reported screening uptake among non-adherent

13 sub-groups of standard samples were used.

14 Heterogeneity between studies was quantified as Cochran's Q (with respective p-value) and $\mathrm{I}^{2}$

15 values. ${ }^{37}$ Funnel-plots were generated to assess the possibility of publication bias influencing the

16 main analyses and Egger's test ${ }^{38}$ and Begg \& Mazumdar's test ${ }^{39}$ were applied to quantify the risk

17 of publication bias. Where either was significant, a trim-and-fill analysis was applied to

18 recalculate the O.R. effect of CBHW interventions after removing all studies which could

19 potentially be introducing publication bias. ${ }^{40}$

20 Sensitivity analyses examined the size of main effects (i) only among trials with full outcome

21 data or intention-to-treat analyses, and (ii) when using only medical record data or self-report

22 measures alone. 
1 Subgroup analyses were used to investigate four potential moderators of overall intervention

2 effect. Firstly, the extent to which effects of CBHW interventions on attendance were found for

3 specific cancer types was examined, specifically breast cancer (mammography), colorectal

4 cancer (any measure due to small number of included studies), or cervical cancer (Pap test),

5 individually. As the BSE and CBE data still related to an outcome of interest (early detection of

6 cancer symptoms), it was also included in this analysis. Secondly, moderator analyses considered

7 which settings may be most effective for delivering CBHW interventions to improve cancer

8 screening adherence; comparing pooled effect sizes of interventions delivered in the home,

9 community, and those delivered by telephone only. A third subgroup analysis explored modes of

10 delivery to assess which may be most effective to improve cancer screening adherence;

11 comparing pooled effect sizes of interventions delivered individually, in a group format and

12 those delivered using a combination approach (individual and group). ${ }^{41}$ Finally, a mixed-effects

13 meta-regression was performed to explore the relationship between two continuous moderators

14 (number of BCTs applied and Theory Coding Scheme score) and intervention effect size.

\section{3. Results}

17 3.1. Search results

18 The searches identified 2493 results; 123 full text versions were retrieved and assessed, of which 1933 articles were eligible for inclusion (screening process in Figure 1).

\section{0 [Figure 1 insert here]}

\section{3.2. Included studies and participant details}


1 Twenty individually randomized controlled trials and 13 cluster RCTs were included; 30 were

2 conducted in the USA, two in a US-Mexico border region and one was conducted in Belgium.

3 The majority of studies focused on female participants from ethnic minorities, with only nine of

4 the eligible studies including participants who were male (mean $=31.3 \%$, range $16.4-50.0)$. The

5 mean age of participants was more than 50 years in 17 studies, 10 studies had a mean age less

6 than 50 years, and six did not specify the average sample age. The average length of follow-up

7 across all studies was six months, ranging from immediately post-intervention to 24 months. For

8 more key study characteristics see Appendix S3.

\section{$9 \quad$ 3.2.1. Selection and training processes}

10 A selection process for the CBHWs was described in $\mathrm{k}=7$ (21\%) studies $^{42-48}$, which included

11 assessment of desirable skills/experience ${ }^{44-46}$, personality characteristics ${ }^{42,43,45,48}$ and

12 practicalities such as availability. ${ }^{43,45,47}$ Gender concordance of CBHWs with the target

13 population was reported in $\mathrm{k}=22(67 \%)$ studies. $^{23-25,42-60}$. CBHWs from the same cultural

14 background as the target audience were selected in $\mathrm{k}=18(55 \%)$ studies. $^{23-25,42,43,48,50,52-54,57-64}$

15 One study included personal experience of breast or cervical cancer as a selection criterion for 16 CBHWs. $^{51}$

17 Some discussion of the training CBHWs received was reported in $\mathrm{k}=27(82 \%)$ studies. ${ }^{23,24,42-60 \text {, }}$

$18 \quad{ }^{62-67}$ The most commonly used pedagogic methodologies for training CBHWs were visual

19 aids/flip charts, practice intervention sessions, and role-playing. A competency assessment

20 measure was described in $\mathrm{k}=18$ (55\%) studies including; ongoing supervision throughout

21 intervention period, practice sessions and a written examination. ${ }^{23,24,42,44,45,47,49,52-55,59,60,62-66}$

22 A form of research skills training including the principle of randomization and the importance of 
1 protocol adherence was reported in $\mathrm{k}=12(36 \%)$ studies. $^{24,42,44,45,49,50,52,53,55,62-64}$ The duration

2 of training was described in $\mathrm{k}=17(52 \%)$ studies and the average duration was 11 hours (range: 4-

324 hours). ${ }^{24,42,44,47-49,51-55,59,60,62,64-66}$ For the majority of studies, training was conducted in one

4 session or a series of sessions before the intervention commenced with only $k=3(9 \%)$ reporting

5 booster, refresher sessions at least once throughout the intervention period. ${ }^{45,55,62}$

$6 \quad$ 3.2.2. Theory application

7 A theory base was explicitly reported (i.e., coded yes to item 5 of Theory Coding Scheme) in

$8 \mathrm{k}=15(45 \%)$ interventions. ${ }^{23,25,43-45,53-55,59,60,62-64,67,68}$ Of these 15 studies, a combination of

9 theories was reported in $\mathrm{k}=8(53 \%)($ range $2-4){ }^{23,25,43,45,54,60,63,64}$ The Transtheoretical Model

10 was applied in $\mathrm{k}=8(53 \%)$ studies and the Health Belief Model was reported in $\mathrm{k}=7$ (47\%)

11 studies. At least one explicit link between an intervention technique and a theory-relevant

12 construct was reported in $\mathrm{k}=6(40 \%)$ interventions. ${ }^{23,25,60,62,64,68}$ At least one explicit link

13 between a theory-relevant construct and an intervention technique was reported in $\mathrm{k}=9(60 \%)$

14 interventions. $^{23,54,55,60,62-64,67,68}$ Theoretically relevant constructs (self-efficacy and perceived

15 severity) were measured pre and post-intervention in $\mathrm{k}=2(6 \%)$ studies. $^{44,55}$ Only one study

16 discussed the results in relation to the theoretical basis of the intervention. ${ }^{55}$ Overall, the Theory

17 Coding Scheme illustrated poor theory application; of the 33 interventions, $\mathrm{k}=18(55 \%)$ scored

18 zero and the highest score was four out of a possible eight. This pattern of results precluded

19 assessment of theory score as a moderator variable.

20

21 Overall, 21 of 93 possible BCTs were present in the interventions included in this review.

22 Interventions incorporated between zero and nine BCTs with a mean of four BCTs in each 
1 intervention. Table 1 demonstrates that the most commonly observed techniques involved

2 providing practical social support, prompts and cues and problem solving. This remained the

3 case when results were limited to the BCTs delivered by the CBHW only and other components

4 such as videos and media campaigns were discounted.

5 [Table 1 insert here]

6 3.2.4. Risk of bias

7

8 Overall, study quality was poor (see Appendix S4). Only one study had a published protocol. ${ }^{67,69}$

9 Therefore the risk of bias for selective reporting was coded high for all remaining studies. Other

10 areas of common bias were reliance on self-reported outcomes, inadequate blinding of key

11 personnel and attrition bias. Attrition rates larger than $25 \%$ were reported in $\mathrm{k}=10(30 \%)$

12 studies. $^{43,44,46,51,55,58,65,68,70,71}$

13 3.3. Effects of lay health worker interventions on cancer screening adherence

14 Results of all meta-analyses are displayed in Table 2. Adherence screening data for the primary

15 outcome was available from a total of 21,990 participants across 30 studies; 11,487 were

16 assigned to CBHW interventions, 10,503 to control conditions. ${ }^{23-25,42-60,62-68,72}$ Random-effects

17 meta-analyses found that CBHW interventions resulted in significantly greater uptake of overall

18 cancer screening (see Figure 2) than control conditions (O.R.=1.90, 95\% CI=1.60-2.26,

$19 \mathrm{p}<0.001)$, although there was considerable heterogeneity among study findings $(\mathrm{Q}=199.9$,

$\left.20 \quad \mathrm{p}<0.01, \mathrm{I}^{2}=85.5 \%\right)$.

21 [Figure 2 insert here] 


\section{$1 \quad 3.4$. Publication bias and sensitivity analyses}

2 Although the risk of publication bias was not statistically significant according to Begg \&

3 Mazumdar's test (Kendall's $\mathrm{t}=0.15, \mathrm{p}=0.24)$, Egger's test did indicate publication bias $(\mathrm{p}=0.007)$.

4 Nonetheless, the Duval and Tweedies trim-and-fill analysis showed that, after removing 12

5 studies which may introduce publication bias, CBHW interventions still significantly improved

6 screening adherence more than control conditions (O.R. $=1.40,95 \%$ C.I. $=1.15-1.69)$.

7 A sensitivity analysis was performed on the 19 studies which used ITT analyses or complete

8 outcome data, with 16,300 participants in total. ${ }^{23,42,45-47,49-51,53,54,56-58,62,63,65-67,72}$ This found

9 that CBHW interventions also resulted in increased cancer screening adherence on an intention-

10 to-treat basis (O.R. $=1.98,95 \% \mathrm{CI}=1.57-2.49, \mathrm{p}<0.001)$ again with significant heterogeneity

11 between studies $\left(\mathrm{Q}=146.2, \mathrm{p}<0.01, \mathrm{I}^{2}=87.7 \%\right)$.

12 Further sensitivity analyses showed that $\mathrm{CBHW}$ interventions resulted in significantly greater

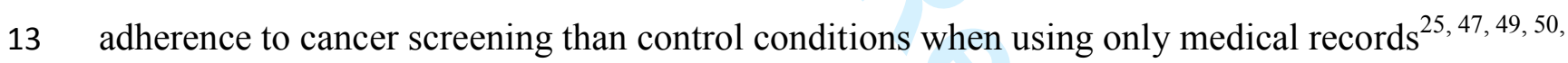

$1456,58,66,72(\mathrm{~N}=8, \mathrm{n}=11658, \mathrm{O} . \mathrm{R} .=2.092,95 \% \mathrm{CI}=1.36-3.21, \mathrm{p}=0.001)$ or self-report data ${ }^{24,25,42,43,}$

$1546,48,51,52,55-60,62-64,68(\mathrm{~N}=18, \mathrm{n}=8620$, O.R. $=1.686,95 \% \mathrm{CI}=1.40-2.03, \mathrm{p}<0.001)$.

16 3.5. Effects of lay health worker interventions on cancer screening uptake in non-adherent

17 samples

18 We also examined the effect of CBHW interventions on uptake of any cancer screening among

19 non-adherent samples (see Appendix S5). Across 20 studies with 12,769 previously non-

20 adherent individuals, LHW interventions resulted in significantly greater likelihood of receiving

21 cancer screening at follow-up compared to control conditions ${ }^{23,24,42,44-49,51,53-55,57,58,65-68,72}$ 
1 (O.R. $=2.40,95 \%$ C.I. $=1.85-3.11, \mathrm{p}<0.001)$. However, there was statistical evidence of both

2 between-study heterogeneity $\left(\mathrm{Q}=149.1, \mathrm{p}<0.01, \mathrm{I}^{2}=87.3 \%\right)$ and publication bias (Egger's $\mathrm{t}=2.33$,

$3 \mathrm{p}=0.03$ ). Nonetheless, the results still significantly favoured LHW interventions after applying

4 the trim-and-fill analysis $(\mathrm{N}=9$, O.R. $=1.58,95 \%$ C.I. $=1.19-2.09)$.

5 3.6. Factors associated with intervention efficacy

6 Further analyses were performed to examine effects of CBHW interventions on the primary

7 outcome (i.e. adherence to cancer screening) when (i) focusing on specific cancer screening

8 types, (ii) comparing different settings of delivery, (iii) comparing different modes of delivery,

9 and (iv) number of BCTs used in interventions (see Table 2).

[Table 2 insert here]

11 Significant benefits of CBHW interventions were observed across each type of screening

12 measure, including mammography $(\mathrm{k}=13, \mathrm{n}=9641, \mathrm{O} . \mathrm{R} .=1.923,95 \% \mathrm{CI}=1.44-2.57, \mathrm{p}<0.001)$,

13 Pap testing $(\mathrm{k}=15, \mathrm{n}=5276, \mathrm{O} . \mathrm{R} .=1.922,95 \% \mathrm{CI}=1.39-2.64, \mathrm{p}<0.001)$ and colorectal cancer

14 screening $(k=7, n=9425$, O.R. $=1.983,95 \% \mathrm{CI}=1.31-3.01, \mathrm{p}<0.001)$. Furthermore, the benefits of

15 CBHW interventions were demonstrated for both BSE $(\mathrm{k}=4, \mathrm{n}=1493$, O.R. $=1.554,95 \%$ CI $=1.17$ -

16 2.07, $\mathrm{p}=0.003)$ and $\mathrm{CBE}(\mathrm{N}=5, \mathrm{n}=2582, \mathrm{O} . \mathrm{R} .=1.528,95 \% \mathrm{CI}=1.19-2.00, \mathrm{p}=0.001)$.

17 Twenty-six studies used home or community settings, and found CBHW interventions were

18 effective when delivered in the home $(\mathrm{k}=13, \mathrm{n}=5779, \mathrm{O} . \mathrm{R} .=1.808,95 \% \mathrm{CI}=1.40-2.34, \mathrm{p}<0.001)$

19 or the community $(\mathrm{k}=13, \mathrm{n}=5621, \mathrm{O} . \mathrm{R} .=2.249,95 \% \mathrm{CI}=1.63-3.11, \mathrm{p}<0.001)$. Four studies

20 examined telephone-only interventions, which demonstrated an overall benefit for CBHWs $(\mathrm{k}=4$,

$21 \mathrm{n}=10590$, O.R. $=1.322,95 \% \mathrm{CI}=1.14-1.54, \mathrm{p}<0.001)$. 
1 Regarding mode of delivery, CBHW interventions were equally as effective when delivered

2 individually $(\mathrm{k}=16, \mathrm{n}=15256, \mathrm{O} . \mathrm{R} .=1.680,95 \% \mathrm{CI}=1.40-2.02, \mathrm{p}<0.001)$ or in a group format

$3(\mathrm{k}=5, \mathrm{n}=2378$, O.R. $=1.633,95 \% \mathrm{CI}=1.36-1.96, \mathrm{p}<0.001$. A combined delivery approach

4 (individual and group elements present) was found to be most effective $(k=8, n=3636$,

5 O.R. $=2.463,95 \% \mathrm{CI}=1.43-4.23, \mathrm{p}=0.001)$.

6 Mixed-effects meta-regression found that number of BCTs used in CBHW interventions held a

7 trend-level association with observed effect size (see Appendix S6); as the O.R. for cancer

8 screening adherence from CBHW interventions increased with the number of BCTs used (B

$9=0.061$, S.E. $=0.036, Z=1.71, p=0.088$ ). A post-hoc subgroup analysis was conducted to

10 examine the effects of CBHW interventions which used a high number of BCTs (four or more)

11 vs. those which used a low number of BCTs (three or less) (Table 2; see Appendix S7). These

12 cut offs were selected in light of the average intervention containing four BCTs. CBHW

13 interventions with a high number of BCTs increased cancer screening with an odds ratio of 2.27

$14(\mathrm{k}=13, \mathrm{n}=6557,95 \%$ C.I. $=1.78-2.89, \mathrm{p}<0.001)$, whereas those with low use of BCTs increased

15 cancer screening by only O.R. $=1.66(\mathrm{k}=17, \mathrm{n}=15433,95 \%$ C.I. $=1.34-2.05, \mathrm{p}<0.001)$, although

16 the difference between High and Low BCT subgroups fell short of statistical significance

$17 \quad(\mathrm{Q}=3.613, \mathrm{p}=0.057)$.

18 The finding of poor theory application precluded assessment of theory score as a moderator

19 variable.

20

21

22 


\section{4. Discussion}

\section{4.1. Summary of main findings}

3 The results from this systematic review and meta-analysis indicate that CBHW interventions are

4 associated with a statistically significant increase in cancer screening attendance, with a stronger

5 effect size observed for participants previously non-adherent with recommended schedules of

6 cancer screening. Similar odds ratios were found for screening for breast, cervical and colorectal

7 cancer. The overall pattern of results was robust to sensitivity analyses, with similar effect sizes

8 found irrespective of source of outcome measure (self-report or medical records), or whether

9 intention-to-treat analyses were conducted or not. There was little use of theory in these

10 interventions, making it difficult to identify the means by which the interventions changed

11 screening attendance. The interventions generally did not use many BCTs, in line with the

12 apparent lack of use of theory to develop interventions. Methodological quality of included

13 studies was generally poor.

15 4.2. Strengths \& limitations

17 The findings of this review build upon the results of previous smaller reviews examining

18 mammography screening only. ${ }^{11,22}$ This review has been the first to illustrate the effectiveness

19 of CBHW interventions across all types of cancer screening and other measures of early

20 detection (BSE and $\mathrm{CBE}$ ). This allows the present review to draw conclusions relating to the

21 efficacy of CBHW interventions across screening for breast, cervical and colorectal cancer.

22 Further the present review has been the first to apply the BCT taxonomy and Theory Coding

23 Scheme to cancer specific CBHW interventions. 
1 A limitation of the present review was that, as many terms are used to describe CBHWs, it may

2 be possible that we did not extract all relevant articles in the existing literature. However, to

3 avoid this, a systematic, comprehensive electronic search was conducted using 45 different terms

4 for CBHW after a consultation with a trained health science librarian. The present review

$5 \quad$ identified many more studies than did previous systematic reviews, and included studies

6 identified by these previous reviews, indicating that this was not a major problem.

7

8 There were however, limitations of the primary evidence base that the present review has

9 identified, but which limit the conclusions that can be drawn. First, substantial heterogeneity

10 was found across the analyses. This between-study heterogeneity is unsurprising, given the clear

11 differences between CBHW interventions in terms of the intervention components used, cancers

12 screened for, guidelines in place at screening, populations studied and outcome measures

13 applied, all sources of heterogeneity which are typical to meta-analytic research. Although this

14 heterogeneity was statistically accounted for by the random-effects model applied, it is

15 acknowledged that the strength of the conclusions from this review are limited as a result.

16 Second, the research base itself was limited to cancer screening outcomes and heterogeneity

17 prevented the combination of secondary outcome data into meaningful categories to examine

18 effectiveness of CBHW interventions in other areas of early cancer diagnosis awareness (e.g.,

19 knowledge, intention, self-efficacy). It was also notable that all but one of the included studies

20 were implemented in the USA and therefore caution is needed to generalize and apply findings

21 to countries with different healthcare systems, cancer screening guidelines and ethnic

22 compositions. 
1 The review identified several methodological biases present in the primary studies; high rates of

2 attrition, reliance on self-reported outcomes, and failure to report blinding which could have

3 contributed to biased estimates of interventional efficacy. However it is important to note that

4 there is an inherent difficulty with blinding in trials assessing the effectiveness of CBHWs. In the

5 majority of cases CBHWs recruit participants from their own social networks/communities as

6 shared characteristics between the CBHW and the audience facilitate learning and increase the

7 likelihood that participants will model the desired behaviour. ${ }^{73}$ Therefore blinding may interfere

8 with one of the presumed mechanisms of effectiveness. Nevertheless, sensitivity analyses

9 suggested that these methodological limitations did not unduly affect the overall conclusions.

10 Furthermore, publication bias was indicated but after removing studies with smaller sample

11 sizes, improvements in screening attendance in CBHW conditions remained significant.

\section{4.3. Interpretation of main findings}

15 This study has been the first to illustrate the effectiveness of CBHW interventions beyond 16 mammography screening. Furthermore this meta-analysis yielded higher odds ratios than

17 previous reviews (1.90-2.40), indicating larger effects of CBHW interventions than previous

18 reviews have suggested. ${ }^{11,22}$ Subgroup analyses revealed similar effects for breast, cervical and 19 colorectal cancer screening.

20

21 The review provides an updated description of $\mathrm{CBHW}$ training characteristics, crucial to the

22 successful implementation of CBHW interventions. ${ }^{74}$ Marked heterogeneity was present in the 23 reporting of selection and training processes in $\mathrm{CBHW}$ literature. A conceptual model has been 
1 proposed to standardize the reporting of such processes in the future. ${ }^{75}$ This transparency will

2 facilitate learning for future intervention development. However, how training content is selected

3 remains unclear, and the lack of readily reported information about components of training

4 hinders the development of evidence based training packages.

5

6 The present review explored potential moderators of effectiveness to provide practical

7 recommendations for future CBHW intervention development. Moderator analyses demonstrated

8 that CBHW interventions were equally as effective across all types of cancer screening.

9 Subgroup analyses revealed that interventions were effective when delivered in community and

10 home settings, over the telephone and when both individual and group modes of delivery were

11 used. Only one of the included interventions was delivered in a primary care setting. ${ }^{70}$ However

12 as it measured knowledge changes only, there was no eligible data to include in the meta-

13 analysis of effects on screening behaviour. No beneficial effect of the CBHW was found when

14 compared to usual care but additional research is needed to establish whether delivering a

15 CBHW intervention in primary care settings has the potential to be effective.

16

17 An in-depth analysis with Theory Coding Scheme items ${ }^{31}$ revealed substantial evidence of

18 'theory inspired' interventions, whereby theory is not used to identify methods to change

19 behaviour, as evidenced by poor linkage between BCTs and theoretical constructs. ${ }^{76}$ This

20 absence of an explicit theoretical basis for interventions makes it difficult to identify why

21 interventions worked, and how future interventions could work better: it prevents an

22 accumulation of knowledge on what basis future CBHW interventions should be developed. ${ }^{77},{ }^{78}$

23 Few BCTs were generally used: CBHWs were found to most frequently provide practical 
1 support to participants, resolve barriers and prompt the desired behaviour. There was a trend

$2(p=0.057)$ towards the use of BCTs being positively associated with intervention effectiveness;

3 larger effect sizes were observed in studies using more than four BCTs $(\mathrm{OR}=2.27)$ compared to

4 those using three or fewer $(\mathrm{OR}=1.66)$. Given that few BCTs were generally used, this would

5 have reduced the association between BCTs and effectiveness, due to a lack of range. Given this,

6 at the least the inclusion of more BCTs should be considered in future studies, to examine

7 whether this results in increased intervention effectiveness.

9 Of note was the exclusive emphasis on cancer screening behaviour observed in this review. Half

10 of the included studies measured secondary outcomes related to knowledge but this was limited

11 to awareness of screening procedures, the benefits of early diagnosis, and cancer risk factors.

12 International comparisons have reported greater endorsement of barriers to help-seeking in UK

$13 \operatorname{adults}^{79}$, in particular regarding the perception of not wanting to waste the doctor's time or cause

14 a fuss. ${ }^{80}$ Despite public awareness campaigns increasing cancer symptom awareness, GP

15 approachability remains a barrier to early engagement with primary care ${ }^{8}$, and a lack of

16 engagement is more pronounced in lower socioeconomic groups. ${ }^{81}$ This suggests that broadening

17 the $\mathrm{CBHW}$ remit to increasing symptom recognition and promotion of help-seeking behaviour is

18 a worthwhile avenue to pursue, particularly in a UK context where barriers to help-seeking

19 remain high.

20

21 4.4. Implications and future research directions

22 This review has demonstrated that $\mathrm{CBHW}$ interventions are a promising approach to addressing

23 cancer screening disparities in the US, particularly in ethnic minority groups. Notwithstanding 
1 limitations in evidence base, the evidence and size of effect obtained in this review support the

2 implementation of CBHW interventions to increase cancer screening in ethnic minorities. Based

3 on the current evidence base, those previously non-adherent with recommended schedules of

4 cancer screening are most likely to benefit.

5 However substantial work is still needed to fully elucidate the conditions key to intervention

6 effectiveness using methodologically sound studies in different populations. As such, the

7 following recommendations for future research are made:

8 a) Guidelines such as the Template for intervention description and replication (TIDieR) ${ }^{82}$ should be used more to increase transparency and facilitate learning between researchers. This would contribute to the identification of key mechanisms of behaviour change, the most effective components of training packages and reduce heterogeneity prevalent across studies.

b) Use of explicit theory in the development and evaluation of interventions, to target hypothetical mediators of behaviour change, such as knowledge and intent, to increase intervention effectiveness. The use of theory in evaluation of such interventions should clarify the processes of change initiated by CBHWs. This would help inform the need for refinement in terms of intervention components and BCTs.

c) The use of a greater range of BCTs should be considered in future interventions, to allow an assessment of whether this would result in greater intervention effectiveness, compared to $\mathrm{CBHW}$ interventions conducted to date. 
1

2

3

4

5

6

7

\section{5. Conclusions}

9 In conclusion, this review has found that $\mathrm{CBHWs}$ are an effective resource for increasing cancer

10 screening uptake, particularly for those previously non-adherent with recommended schedules of

11 cancer screening. It is yet unknown whether CBHW interventions are an effective approach for

12 other areas of early diagnosis including symptom awareness and help-seeking behaviour. The

13 number of explicitly described BCTs used was found to be positively associated with effect size,

14 although this did not reach statistical significance. At present, these conclusions are almost

15 entirely limited to the US healthcare context and ethnic composition and limited by poor

16 methodological quality and substantial heterogeneity. As such it remains unknown whether the

17 effectiveness of CBHW interventions would translate to different countries or other areas

18 pertinent to early cancer diagnosis such as help-seeking behaviour.

\section{Conflicts of interest}

20

21 The authors declare no potential conflicts of interest. 
References

References marked with an asterisk indicate studies included in the systematic review

1. World Health Organization. Global action plan for the prevention and control of NCDs 20132020 [Internet]. World Health Organization; 2013. Available from: http://apps.who.int/iris/bitstream/10665/94384/1/9789241506236 eng.pdf?ua=1

2. World Health Organization. Guide to cancer early diagnosis [Internet]. World Health Organization; 2017. Available from:

http://apps.who.int/iris/bitstream/10665/254500/1/9789241511940-eng.pdf?ua=1 3. Ott JJ, Ullrich A, Miller AB. The importance of early symptom recognition in the context of early detection and cancer survival. Eur J Cancer. 2009;45:2743-8

4. CRUK. Cancer Awareness Measure (CAM) Key Findings Report; 2014 \& Trends Analysis (2008-2014) [Internet]. 2014. Available from: http://www.cancerresearchuk.org/sites/default/files/cam key findings report 2014 trends analysis_v5.pdf

5. Health and Social Care Information Centre. Cervical Screening Programme - England, 201415 [Internet]. National Statistics; 2015. Available from: http://content.digital.nhs.uk/catalogue/PUB18932/nhs-cervical-stat-eng-2014-15-rep.pdf 6. Health and Social Care Information Centre. Breast Screening Programme - England, 2014-15 [Internet]. National Statistics; 2016. Available from: http://content.digital.nhs.uk/catalogue/PUB20018/bres-scre-prog-eng-2014-15-rep.pdf 7. Smith RA, Manassaram-Baptiste D, Brooks D, Doroshenk M, Fedewa S, Saslow D, et al. Cancer screening in the United States, 2015: A review of current American Cancer Society guidelines and current issues in cancer screening. CA Cancer J Clin. 2015;65:30-54

8. Power E, Wardle J. Change in public awareness of symptoms and perceived barriers to seeing a doctor following Be Clear on Cancer campaigns in England. Br J Cancer. 2015;112:S22-S6

9. Guessous I, Dash C, Lapin P, Doroshenk M, Smith RA, Klabunde CN. Colorectal cancer screening barriers and facilitators in older persons. Prev Med. 2010;50:3-10 10. Smith LK, Pope C, Botha JL. Patients' help-seeking experiences and delay in cancer presentation: a qualitative synthesis. The Lancet. 2005;366:825-31

11. Lewin SA, Dick J, Pond P, Zwarenstein M, Aja G, van Wyk B, et al. Lay health workers in primary and community health care. The Cochrane database of systematic reviews. 2005. CD004015.

12. Witmer A, Seifer SD, Finocchio L, Leslie J, O'Neil EH. Community health workers: integral members of the health care work force. Am J Public Health. 1995;85:1055-8 13. Hou S-I, Roberson K. A Systematic Review on US-Based Community Health Navigator (CHN) Interventions for Cancer Screening Promotion - Comparing Community- Versus ClinicBased Navigator Models. J Cancer Educ. 2015;30:173-86 14. Rhodes SD, Foley KL, Zometa CS, Bloom FR. Lay Health Advisor Interventions Among Hispanics/Latinos: A Qualitative Systematic Review. Am J Prev Med. 2007;33:418-27 15. Freeman HP. A model patient navigation program. Oncology Issues. 2004;19:44-6 16. Wells KJ, Battaglia TA, Dudley DJ, Garcia R, Greene A, Calhoun E, et al. Patient navigation: State of the art or is it science? Cancer. 2008;113:1999-2010 17. Gibbons MC, Tyus NC. Systematic review of U.S.-based randomized controlled trials using community health workers. Prog Community Health Partnersh. 2007;1:371-81 
18. Islam NS, Zanowiak JM, Riley L, Nadkarni SK, Kwon SC, Trinh-Shevrin C. Characteristics of Asian American, Native Hawaiian, and Pacific Islander community health worker programs: a systematic review. J Health Care Poor Underserved. 2015;26:238-68 19. Viswanathan M, Kraschnewski JL, Nishikawa B, Morgan LC, Honeycutt AA, Thieda P, et al. Outcomes and Costs of Community Health Worker Interventions: A Systematic Review. Med Care. 2010;48:792-808

20. Wadler BM, Judge CM, Prout M, Allen JD, Geller AC. Improving Breast Cancer Control via the Use of Community Health Workers in South Africa: A Critical Review. J Oncol. 2011;2011:H382-7

21. Kim K, Choi JS, Choi E, Nieman CL, Joo JH, Lin FR, et al. Effects of Community-Based Health Worker Interventions to Improve Chronic Disease Management and Care Among Vulnerable Populations: A Systematic Review. Am J Public Health. 2016;106:e3-e28 22. Wells KJ, Luque JS, Miladinovic B, Vargas N, Asvat Y, Roetzheim RG, et al. Do Community Health Worker Interventions Improve Rates of Screening Mammography in the United States? A Systematic Review. Cancer Epidemiol Biomarkers Prev. 2011;20:1580-98 23. *Byrd TL, Wilson KM, Smith JL, Coronado G, Vernon SW, Fernandez-Esquer ME, et al. AMIGAS: A multicity, multicomponent cervical cancer prevention trial among Mexican American women. Cancer. 2013;119:1365-72

24. *Nguyen BH, Stewart SL, Nguyen TT, Bui-Tong N, McPhee SJ. Effectiveness of Lay Health Worker Outreach in Reducing Disparities in Colorectal Cancer Screening in Vietnamese Americans. Am J Public Health. 2015; 105:2083-9 25. *Paskett ED, McLaughlin JM, Lehman AM, Katz ML, Tatum CM, Oliveri JM. Evaluating the Efficacy of Lay Health Advisors for Increasing Risk-Appropriate Pap Test Screening: A Randomized Controlled Trial among Ohio Appalachian Women. Cancer Epidemiol Biomarkers Prev. 2011;20:835-43

26. Michie S, Richardson M, Johnston M, Abraham C, Francis J, Hardeman W, et al. The Behavior Change Technique Taxonomy (v1) of 93 Hierarchically Clustered Techniques: Building an International Consensus for the Reporting of Behavior Change Interventions. Ann Behav Med. 2013;46:81-95

27. French DP, Olander EK, Chisholm A, Mc Sharry J. Which Behaviour Change Techniques Are Most Effective at Increasing Older Adults' Self-Efficacy and Physical Activity Behaviour? A Systematic Review. Ann Behav Med. 2014;48:225-34

28. Moher D, Liberati A, Tetzlaff J, Altman DG, Group P. Preferred reporting items for systematic reviews and meta-analyses: the PRISMA statement. PLoS Med. 2009;6: 1000097 29. Evans D. Hierarchy of evidence: a framework for ranking evidence evaluating healthcare interventions. J Clin Nurs. 2003; 12:77-84

30. Wood CE, Richardson M, Johnston M, Abraham C, Francis J, Hardeman W, et al. Applying the behaviour change technique (BCT) taxonomy v1: a study of coder training. Transl Behav Med. 2015;5:134-48

31. Michie S, Prestwich A. Are interventions theory-based? Development of a theory coding scheme. Health Psychol. 2010;29:1-8

32. Prestwich A, Sniehotta FF, Whittington C, Dombrowski SU, Rogers L, Michie S. Does theory influence the effectiveness of health behavior interventions? Meta-analysis. Health Psychol. 2014;33:465-74 
33. Janz NK, Becker MH. The Health Belief Model: A Decade Later. Health Educ Q. 1984;11:147

34. Higgins JPT, Altman DG, Gøtzsche PC, Jüni P, Moher D, Oxman AD, et al. The Cochrane Collaboration's tool for assessing risk of bias in randomised trials. BMJ. 2011;343:d5928

35. Borenstein M, Hedges L, Higgins J, Rothstein H. Comprehensive Meta Analysis. 2 ed. Englewood, NJ: Biostat; 2005.

36. DerSimonian R, Kacker R. Random-effects model for meta-analysis of clinical trials: An update. Contemp Clin Trials. 2007;28:105-14

37. Higgins JPT, Thompson SG. Quantifying heterogeneity in a meta-analysis. Stat Med.

2002;21:1539-58

38. Egger M, Smith GD, Schneider M, Minder C. Bias in meta-analysis detected by a simple, graphical test. $B M J$. 1997;315:629-34

39. Begg CB, Mazumdar M. Operating Characteristics of a Rank Correlation Test for Publication Bias. Biometrics. 1994;50:1088-101

40. Duval S, Tweedie R. Trim and Fill: A Simple Funnel-Plot-Based Method of Testing and Adjusting for Publication Bias in Meta-Analysis. Biometrics. 2000;56:455-63

41. Dombrowski SU, O'Carroll RE, Williams B. Form of delivery as a key 'active ingredient' in behaviour change interventions. Br J Health Psychol. 2016;21:733-40

42. *Lam TK, Mc Phee SJ, Mock J, Wong C, Doan HT, Nguyen T, et al. Encouraging

Vietnamese-American Women to Obtain Pap Tests Through Lay Health Worker Outreach and Media Education. J Gen Intern Med. 2003; 18:516-24

43. *Navarro AM, Senn KL, McNicholas LJ, Kaplan RM, Roppé B, Campo MC. Por La Vida model intervention enhances use of cancer screening tests among Latinas. Am J Prev Med.

1998; $15: 32-41$

44. *O'Brien MJ, Halbert CH, Bixby R, Pimentel S, Shea JA. Community Health Worker Intervention to Decrease Cervical Cancer Disparities in Hispanic Women. J Gen Intern Med. 2010; $25: 1186-92$

45. *Paskett E, Tatum C, Rushing J, Michielutte R, Bell R, Foley KL, et al. Randomized Trial of an Intervention to Improve Mammography Utilization Among a Triracial Rural Population of Women. J Natl Cancer Inst. 2006;98:1226-37

46. *Sung JF, Blumenthal DS, Coates RJ, Williams JE, Alema-Mensah E, Liff JM. Effect of a cancer screening intervention conducted by lay health workers among inner-city women. $\mathrm{Am} J$ Prev Med. 1997; 13:51-7

47. *Han H-R, Song Y, Kim M, Hedlin HK, Kim K, Ben Lee H, et al. Breast and Cervical Cancer Screening Literacy Among Korean American Women: A Community Health WorkerLed Intervention. Am J Public Health. 2017;107:159-65

48. *Mock J, McPhee SJ, Nguyen T, Wong C, Doan H, Lai KQ, et al. Effective Lay Health Worker Outreach and Media-Based Education for Promoting Cervical Cancer Screening Among Vietnamese American Women. Am J Public Health. 2007;97:1693-700

49. *Goelen G, De Clercq G, Hanssens S. A community peer-volunteer telephone reminder call to increase breast cancer-screening attendance. Oncol Nurs Forum. 2010;37:E312-7

42 50. *Hunter JB, de Zapien JG, Papenfuss M, Fernandez ML, Meister J, Giuliano AR. The Impact 43 of a Promotora on Increasing Routine Chronic Disease Prevention among Women Aged 40 and

44 Older at the U.S.-Mexico Border. Health Educ Behav. 2004;31:18S-28S 
51. *Jandorf L, Bursac Z, Pulley L, Trevino M, Castillo A, Erwin DO. Breast and cervical cancer screening among Latinas attending culturally specific educational programs. Prog Community Health Partnersh. 2008;2:195-204 52. *Nguyen TT, Le G, Nguyen T, Le K, Lai K, Gildengorin G, et al. Breast Cancer Screening Among Vietnamese Americans: A Randomized Controlled Trial of Lay Health Worker Outreach. Am J Prev Med. 2009;37:306-13 53. *Nuño T, Martinez ME, Harris R, García F. A Promotora-administered group education intervention to promote breast and cervical cancer screening in a rural community along the U.S.-Mexico border: a randomized controlled trial. Cancer Causes Control. 2011;22:367-74 54. *Russell KM, Champion VL, Monahan PO, Millon-Underwood S, Zhao Q, Spacey N, et al. Randomized Trial of a Lay Health Advisor and Computer Intervention to Increase Mammography Screening in African American Women. Cancer Epidemiol Biomarkers Prev. 2010; $19: 201-10$ 55. *Sadler GR, Ko CM, Wu P, Alisangco J, Castañeda SF, Kelly C. A Cluster Randomized Controlled Trial to Increase Breast Cancer Screening Among African American Women: The Black Cosmetologists Promoting Health Program. J Natl Med Assoc. 2011;103:735-45 56. *Taylor VM, Carey Jackson J, Yasui Y, Kuniyuki A, Acorda E, Marchand A, et al. Evaluation of an outreach intervention to promote cervical cancer screening among Cambodian American women. Cancer Detect Prev. 2002a;26:320-7 57. *Taylor VM, Hislop TG, Jackson JC, Tu S-P, Yasui Y, Schwartz SM, et al. A Randomized Controlled Trial of Interventions to Promote Cervical Cancer Screening Among Chinese Women in North America. J Natl Cancer Inst. 2002b;94:670-7 58. *Taylor VM, Jackson JC, Yasui Y, Nguyen TT, Woodall E, Acorda E, et al. Evaluation of a Cervical Cancer Control Intervention Using Lay Health Workers for Vietnamese American Women. Am J Public Health. 2010;100:1924-9 59. *Walsh JME, Salazar R, Nguyen TT, Kaplan C, Nguyen L, Hwang J, et al. Healthy Colon, Healthy Life: A Novel Colorectal Cancer Screening Intervention. Am J Prev Med. 2010;39:1-14 60. *Zhu K, Hunter S, Bernard LJ, Payne-Wilks K, Roland CL, Elam LC, et al. An Intervention Study on Screening for Breast Cancer among Single African-American Women Aged 65 and Older. Prev Med. 2002;34:536-45

61. *Carney PA, Lee-Lin F, Mongoue-Tchokote S, Mori M, Leung H, Lau C, et al. Improving colorectal cancer screening in Asian Americans: Results of a randomized intervention study. Cancer. 2014; $120: 1702-12$ 62. *Nguyen TT, Tsoh JY, Woo K, Stewart SL, Le GM, Burke A, et al. Colorectal Cancer Screening and Chinese Americans: Efficacy of Lay Health Worker Outreach and Print Materials. Am J Prev Med. 2017;52:e67-e76 63. *Tong EK, Nguyen TT, Lo P, Stewart SL, Gildengorin GL, Tsoh JY, et al. Lay health educators increase colorectal cancer screening among Hmong Americans: A cluster randomized controlled trial. Cancer. 2017;123:98-106 64. *Jo AM, Nguyen TT, Stewart S, Sung MJ, Gildengorin G, Tsoh JY, et al. Lay health educators and print materials for the promotion of colorectal cancer screening among Korean Americans: A randomized comparative effectiveness study. Cancer. 2017. Epub 2017 Apr 25 65. *Fernández ME, Gonzales A, Tortolero-Luna G, Williams J, Saavedra-Embesi M, Chan W, et al. Effectiveness of Cultivando La Salud: A Breast and Cervical Cancer Screening Promotion Program for Low-Income Hispanic Women. Am J Public Health. 2009;99:936-43 
66. *Percac-Lima S, Grant RW, Green AR, Ashburner JM, Gamba G, Oo S, et al. A Culturally Tailored Navigator Program for Colorectal Cancer Screening in a Community Health Center: A Randomized, Controlled Trial. J Gen Intern Med. 2009;24:211-7

67. *Thompson B, Carosso EA, Jhingan E, Wang L, Holte SE, Byrd TL, et al. Results of a randomized controlled trial to increase cervical cancer screening among rural Latinas. Cancer. 2017; $123: 666-74$

68. *Duan N, Fox SA, Derose KP, Carson S. Maintaining mammography adherence through telephone counseling in a church-based trial. Am J Public Health. 2000;90:1468-71

69. Duggan C, Coronado G, Martinez J, Byrd TL, Carosso E, Lopez C, et al. Cervical cancer screening and adherence to follow-up among Hispanic women study protocol: a randomized controlled trial to increase the uptake of cervical cancer screening in Hispanic women. BMC Cancer. 2012;12:170

70. *Castañeda SF, Xiong Y, Gallo LC, Yepes-Rios M, Ji M, Talavera AC, et al. Colorectal Cancer Educational Intervention Targeting Latino Patients Attending a Community Health Center. J Prim Care Community Health. 2012;3:164-9

71. *Sadler GR, Beerman PR, Lee K, Hung J, Nguyen H, Cho J, et al. Promoting Breast Cancer Screening Among Asian American Women: the Asian Grocery Store-Based Cancer Education Program. J Cancer Educ. 2012;27:612-7

72. *Potter MB, Namvargolian Y, Hwang J, Walsh JME. Improving Colorectal Cancer Screening: A Partnership Between Primary Care Practices and the American Cancer Society. $J$ Cancer Educ. 2009;24:22-7

73. Bandura. Social cognitive theory. Annals of Child Development. 1989;6:1-66

74. Swider SM, Martin M, Lynas C, Rothschild S. Project MATCH. Diabetes Educ. 2010;36:98108

75. O'Brien MJ, Squires AP, Bixby RA, Larson SC. Role Development of Community Health Workers: An Examination of Selection and Training Processes in the Intervention Literature. $\mathrm{Am}$ J Prev Med. 2009;37:S262-S9

76. Michie S, Johnston M, Francis J, Hardeman W, Eccles M. From Theory to Intervention: Mapping Theoretically Derived Behavioural Determinants to Behaviour Change Techniques. Appl Psychol. 2008;57:660-80

77. Michie S, Johnston M. Theories and techniques of behaviour change: Developing a cumulative science of behaviour change. Health Psychol Rev. 2012;6:1-6

78. Foy R, Eccles M, Jamtvedt G, Young J, Grimshaw J, Baker R. What do we know about how to do audit and feedback? Pitfalls in applying evidence from a systematic review. BMC Health Serv Res. 2005;5:50

79. Forbes LJL, Simon AE, Warburton F, Boniface D, Brain KE, Dessaix A, et al. Differences in cancer awareness and beliefs between Australia, Canada, Denmark, Norway, Sweden and the UK (the International Cancer Benchmarking Partnership): do they contribute to differences in cancer survival[quest]. Br J Cancer. 2013;108:292-300

80. Whitaker KL, Macleod U, Winstanley K, Scott SE, Wardle J. Help seeking for cancer 'alarm' symptoms: a qualitative interview study of primary care patients in the UK. Br J Gen Pract. 2015;65:e96-e105

81. Moffat J, Bentley A, Ironmonger L, Boughey A, Radford G, Duffy S. The impact of national cancer awareness campaigns for bowel and lung cancer symptoms on sociodemographic 
1 inequalities in immediate key symptom awareness and GP attendances. Br J Cancer.

$2 \quad 2015 ; 112: \mathrm{S} 14-\mathrm{S} 21$

3 82. Hoffmann TC, Glasziou PP, Boutron I, Milne R, Perera R, Moher D, et al. Better reporting of

4 interventions: template for intervention description and replication (TIDieR) checklist and guide.

5 BMJ. 2014;348:g1687

6

7

$13-7$

14

15

16

17

18

19

20

21

22

23

24

25

26

27

29

30

31

32

33

34

35
36

37

38

39

40

41

42

43
44

45

46

47

48

49

50

51

52

53

54

55

56

57 
Table 1. Prevalence of behaviour change techniques in community-based health worker interventions

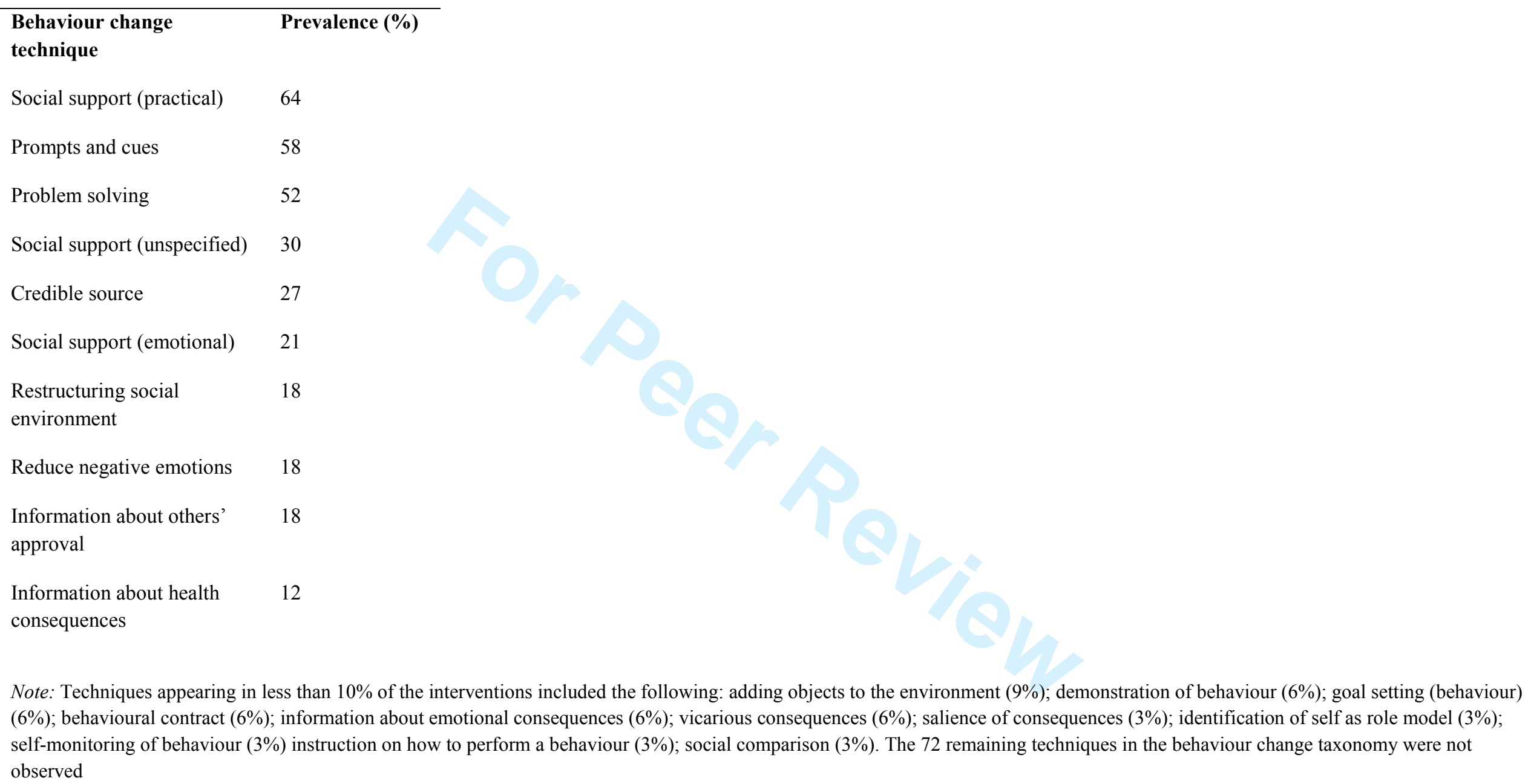


Table 2. Meta-analyses of studies reporting the effect of community-based health worker interventions on cancer screening

\begin{tabular}{|c|c|c|c|c|c|c|c|c|c|}
\hline & \multicolumn{3}{|l|}{ Sample } & \multicolumn{3}{|c|}{ Meta-analysis } & \multicolumn{3}{|c|}{ Heterogeneity } \\
\hline & Studies & Total $n$ & $\begin{array}{l}\text { Odds } \\
\text { Ratio } \\
\end{array}$ & & $\mathbf{6} \mathbf{C I}$ & P value & Q-value & $\mathrm{P}$ value & $\mathrm{I}^{2}$ \\
\hline \multicolumn{10}{|l|}{ Primary and secondary outcomes } \\
\hline Primary: Overall screening adherence & 30 & 21990 & 1.901 & 1.60 & 2.26 & $<0.001$ & 199.9 & $<0.01$ & 85.5 \\
\hline $\begin{array}{l}\text { Secondary: Uptake among non- } \\
\text { adherent }\end{array}$ & 20 & 12768 & 2.401 & 1.85 & 3.11 & $<0.001$ & 149.1 & $<0.01$ & 87.3 \\
\hline \multicolumn{10}{|l|}{ Sensitivity analyses } \\
\hline Intention to treat data only & 19 & 16300 & 1.978 & 1.57 & 2.49 & $<0.001$ & 146.2 & $<0.01$ & 87.7 \\
\hline Medical records data only & 8 & 11658 & 2.092 & 1.36 & 3.21 & 0.001 & 112.6 & $<0.01$ & 93.8 \\
\hline Self-report data only & 18 & 8620 & 1.686 & 1.40 & 2.03 & $<0.001$ & 64.0 & $<0.01$ & 73.4 \\
\hline \multicolumn{10}{|l|}{ Subgroup analyses $=$ type of procedure } \\
\hline Breast cancer (mammography) & 13 & 9641 & 1.923 & 1.44 & 2.57 & $<0.001$ & 96.7 & $<0.01$ & 87.6 \\
\hline Breast cancer (BSE) & 4 & 1493 & 1.554 & 1.17 & 2.07 & 0.003 & 4.96 & 0.175 & 39.5 \\
\hline Breast cancer (CBE) & 5 & 2582 & 1.528 & 1.19 & 2.00 & 0.001 & 8.76 & 0.067 & 54.4 \\
\hline Cervical cancer (pap test) & 15 & 5276 & 1.922 & 1.39 & 2.64 & $<0.001$ & 92.7 & $<0.01$ & 84.9 \\
\hline Colorectal (any) & 7 & 9425 & 1.983 & 1.31 & 3.01 & $<0.001$ & 86.8 & $<0.01$ & 93.1 \\
\hline \multicolumn{10}{|l|}{ Subgroup analyses $=$ delivery setting } \\
\hline Community & 13 & 5621 & 2.249 & 1.63 & 3.11 & $<0.001$ & 83.7 & $<0.01$ & 85.7 \\
\hline Home-based & 13 & 5779 & 1.808 & 1.40 & 2.34 & $<0.001$ & 54.5 & $<0.01$ & 78.0 \\
\hline Telephone & 4 & 10590 & 1.322 & 1.14 & 1.54 & $<\mathbf{0 . 0 0 1}$ & 5.91 & 0.116 & 49.3 \\
\hline
\end{tabular}




\begin{tabular}{|c|c|c|c|c|c|c|c|c|c|}
\hline \multicolumn{10}{|c|}{ Subgroup analyses $=$ intervention features } \\
\hline Individual & 16 & 15256 & 1.680 & 1.40 & 2.02 & $<0.001$ & 63.1 & $<0.01$ & 76.2 \\
\hline Group-based & 5 & 2378 & 1.633 & 1.36 & 1.96 & $<0.001$ & 3.42 & 0.491 & 0.00 \\
\hline Individual + Group & 8 & 3636 & 2.463 & 1.43 & 4.23 & 0.001 & 93.8 & $<0.01$ & 92.5 \\
\hline$B C T s ; 3$ or less & 17 & 15433 & 1.660 & 1.34 & 2.05 & $<0.001$ & 105.5 & 0.001 & 84.8 \\
\hline BCTs; 4 or more & 13 & 6557 & 2.268 & 1.78 & 2.89 & $<0.001$ & 50.057 & 0.001 & 76.0 \\
\hline
\end{tabular}

Note: BSE, breast self-examination; CBE, clinical breast examination; ITT, intention to treat BOLD represents statistically significant benefit of CBHW interventions. 


\section{Figure legends}

2

3

4

5

6

7

8

9

10

11

12

13

14

15

16

17

18

19

20

21

22

23

24

25

26

27

28

29

30

31

32

33

34

35

36

37

38

39

40

41

42

43

44

45

46

47

48

49

50

51

52

53

54

55

56

57

58

59

60

Figure 1. PRISMA flow diagram of study selection

Figure 2. Meta-analysis of cancer screening adherence following CBHW interventions in comparison to control conditions. Box size represents study weighting. Diamond represents overall effect size and $95 \%$ confidence intervals. 
2

Running title: $\mathrm{LCBHW}_{\underline{\mathrm{C}} \overline{\mathrm{C}} \text { and }}$ Early Diagnosis of Cancer and Cancer Prevention

Are community-based lay health worker interventions an effective approach for eancer prevention and early diagnosis of cancer? A systematic review and meta-analysis Sarah Bellhouse ${ }^{1}$, Lorna McWilliams ${ }^{1}$, Joseph Firth ${ }^{2,3}$, Janelle Yorke ${ }^{1,4}$, \& David P. French $^{5}$

\section{Authors' affiliations:}

${ }^{1}$ Christie Patient Centred Research Group, School of Oncology, The Christie NHS Foundation Trust, UK

${ }^{2}$ Division of Psychology and Mental Health, School of Health Sciences, University of Manchester, UK

${ }^{3}$ NICM, School of Science and Health, University of Western Sydney, Australia

${ }^{4}$ Division of Nursing, Midwifery and Social Work, School of Health Sciences, University of Manchester UK

${ }^{5}$ Manchester Centre for Health Psychology, School of Health Sciences, University of Manchester, UK

\section{Corresponding author:}

Sarah Bellhouse, Christie Patient Centred Research Group, School of Oncology, The Christie NHS Foundation Trust, Wilmslow Road, Withington, Manchester, M20 4BX, UK. Phone: 0161 918 2451; E-mail: sarah.bellhouse@christie.nhs.uk

\section{Word count: $\underline{6,259} 6,000$}

Figures/tables: 4 
Abstract

2 Objective: This systematic review aimed to assess the effectiveness of lay community-based

3 health worker (LㅌHW) interventions for early detection of cancer-and cancer prevention.

4 Secondary aims were to consider the extent that interventions were based on theory, and

5 potential moderators including behaviour change techniques (BCTs).

6 Methods: Six databases were searched for randomized controlled trials. Random-effects meta-

7 analyses were applied to 30 eligible studies with a cancer screening outcome.

8

9

Results: Participation in $\mathrm{ECBHW}$ interventions was associated with increased receipt of screening $(\mathrm{OR}=1.901,95 \% \mathrm{CI}: 1.60-2.26, \mathrm{p}<0.001)$ for breast, cervical and bowel cancer.

Larger effect sizes were observed in participants previously non-adherent with recommended schedules of cancer screening. 25/30 studies were conducted with ethnic minority groups. Only $1 \underline{57}(4 \underline{5} 9 \%)$ studies explicitly reported a theoretical foundation for intervention. The number of BCTs used by $\mathrm{ECBHWs}^{\mathrm{CB}}$ had a trend level association with observed effect size $(\mathrm{p}=0.08)$. Study quality was generally poor and common limitations were inadequate blinding and reliance on self-reported outcomes. Only two-studies considered the LHW approach for reducing cancer related lifestyle behaviours.

Conclusions: $\mathrm{E} \underline{\mathrm{CB} H W}$ interventions are an effective resource for increasing uptake of all three types of cancer screening in ethnic minority groups. Those previously non-adherent with

19 recommended schedules of cancer screening benefitted the most from the $\underline{\mathrm{CB} H W}$ approach. However, better quality studies based on more explicit evidence-based theory are needed to optimise the effectiveness of $\underline{\mathrm{ECBHW}}$ interventions on screening uptake. Further research is 
1 needed to ascertain whether $\mathbf{L} \underline{\mathrm{CBHW}}$ s can help promote symptom recognition and help-seeking

Keywords: behaviour change techniques, cancer, eancer prevention, early diagnosis, lay 


\section{1. Background}

3 In 2013, The World Health Organization (WHO) published their global action plan for the

4 prevention and control of non-communicable diseases, including cancer. ${ }^{1}$ They recommend providing community based health promotion-services to reduce behaviouralcancerrelated risk factors including tobaceo-smoking and physical inactivity. However, $\mathrm{tThe}$ WHO recognize that not all cancers are preventable and so health services are urged to focus on

8 diagnosing cancer earlier in order to increase survival rates and reduce costs associated with

9 treating the disease. ${ }^{2}$ To facilitate this, more interventions aimed at 'down-staging' cancers such

10 as increasing uptake of screening programmes are warranted. ${ }^{3}$

11 Even though recognition of cancer signs and symptoms are relatively high ${ }^{4}$, attendance rates for

12 cancer screening have decreased in recent years in the UK and USA. ${ }^{5-7}$ Barriers to attending

13 screening or raising a cancer-related health concern with healthcare professionals remain

14 including; language/cultural barriers, embarrassment/fear and perceptions of wasting doctors'

15 time..$^{8-10}$ Evidence also-suggests that public awareness of the links between cancer and

lifestyle risk behaviours such as excessive alcohol consumption and obesity remains low.

17 One approach to overcoming barriers towards cancer screening and early diagnosis is using

18 volunteer Lay Health Workers (LHWs) i.e., any health worker carrying out functions related to health care delivery who does not have any formal professional of paraprofessional qualifications

11. non-professionals trained to deliver cancer prevention and early detection messages who help-connect the community and healtheare providers. ${ }^{12}$ LHW roles in a cancer context 
1 include providing education to raise cancer awareness and overcoming individual barriers to

2 attending screening. Two LHW models have been proposed in the literature, differentiated by $\underline{\text { setting; community based and clinic/hospital based. }}{ }^{13}$ Although there is overlap in tasks completed by both types of LHW, community-based health workers (CBHWs) act as a liaison $\underline{\text { facilitating initial access between individuals and healthcare settings. }}{ }^{12,14}$ In contrast, LHWs $\underline{\text { based in clinic and hospital settings, commonly referred to as patient navigators, offer guidance }}$

$\underline{\text { to persons with an abnormal cancer screening test or a cancer diagnosis to access the cancer care }}$ system. ${ }^{15,16}$ The present review will focus on CBHWs only as they provide the earliest possible

Several systematic reviews have examined the effects of $\underline{\mathrm{ECB}} \mathrm{HW}$ interventions on cancer outcomes, most commonly cancer screening uptake on cancer sereening uptake ${ }^{13,17-21}$ For instance, Two previous systematic reviews which applied meta-analytic techniques found that LCBHWs can improve breast cancer screening uptake, with small to moderate effects $\boldsymbol{\theta d d s}$ ratios of $1.05^{11}$ and $1.06^{22}$ and 1.27 reported.

Although these previous systematic reviews are useful, they have four five limitations that the present research aims to address. Firstly, the searches in these previous systematic reviews were quantitative estimates of effects on screening attendance have only examined mammography

21 uptake, one of several available screening programmes in many countries. Since then, more

22 recent studies have examined the effects of $\mathrm{ECB} H W$ s on other forms of screening ${ }^{23-25}$, 
1

2

3

4 listing theories mentioned. Consequently, it is difficult to assess the extent to which these

5

6

7

8

9

10

11

12

13

14

15

16

17

18

19

20

21

kinds of screening. Thirdly, previous reviews have not used a rigorous assessment to assess how risk of bias of these studies affects their findings. FourthThirdly, previous reviews have not considered the use of theory underpinning intervention development on effectiveness beyond theories are used in the design, analysis and interpretation of review results. FifthFourth, previous reviews have not assessed how the use of behaviour change techniques (BCTs) may enhance increase cancer prevention and early detection outcomes. BCTs have been defined as “observable, replicable, and irreducible component(s) of an intervention designed to alter or redirect causal processes that regulate behaviour" $(\mathrm{p} 82)^{26}$, and have been shown to moderate the effects of interventions for other behaviours. ${ }^{27}$ Thus, previous reviews have not clearly described the active intervention ingredients that $\mathrm{ECBHW}$ interventions include.

The aim of the present review was to systematically identify and critically review studies that investigate the effectiveness of $\mathrm{ECBHWs}$ in promoting outcomes pertinent to eancer prevention and early cancer diagnosis including screening, symptom recognition, early detection, reducing lifestyle related risk factors and help-seeking behaviour. Specific objectives were to: a) determine the effectiveness of lay health led interventions in targeting outcomes related to early detection of cancer, with the expectation that screening uptake will be the likely focus considering previous reviews in this area. b) identify the extent to which risk of bias may affect intervention effectiveness.

c) assess the extent to which $\mathrm{E} \underline{\mathrm{CB}} \mathrm{HW}$ interventions are underpinned by a theoretical foundation and determine the prevalence and types of BCTs included in interventions. 
1 d) examine potential moderators of intervention effectiveness, including type of screening

2 programme, mode of delivery, intervention setting, and use of BCTs.

3

4 2. Methods

$5 \quad$ 2.1. Search strategy

6 This systematic review followed the PRISMA statement, with no ethical approval required. ${ }^{28}$

7 Electronic database searches were conducted of MEDLINE, Embase, BNI, CINAHL, PsycINFO

8 \& AMED in May 2016, and updated in April 2017. No date restrictions were applied for any

9 searches. A broad search strategy was used to identify relevant articles and variation of index

10 terms across databases was taken into account. For the full search strategy for each database, 11 refer to Appendix S1.

12

13 In addition to database searches, forward and backward citation searches were conducted.

14 Companion papers were retrieved where necessary to ascertain further information about

15 intervention development and content and $\mathrm{ECBHW}$ selection/training.

16

\section{2.2. Eligibility criteria}

18 Only English-language research articles published infrom peer-reviewed journals were included 19 in the review. Studies were included if they met the following criteria:

20 (1) Participants: general population ( $\geq 18$ years). 
1

22 2.3. Study selection

(2) Interventions were delivered by a $\mathbf{L C B H W}$ who did not require formal professional or paraprofessional education/qualifications to complete the role. The role of the CBHW was to facilitate initial engagement with healthcare providers and access to services related to early cancer diagnosis e.g. cancer screening or visiting the GP after identifying a potential cancer symptom. Therefore studies delivered in primary care settings would $\underline{\text { be eligible if the person was attending for a routine visit unrelated to cancer. }} \underline{\text { Studies }}$ whereby CBHWs were providing follow up support for those already engaged with

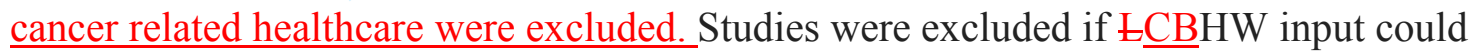
not be assessed where healthcare professionals were also involved. Interventions were included whether the CBHW did or did not receive payment for their work.

(3) Controls: studies had to have at least one non LCBHW comparator arm, with no other restriction placed upon the nature of the comparison with the intervention group.

(4) Outcomes: studies had to measure an outcome that promoted prevention or early diagnosis of cancer at baseline and post-intervention e.g. uptake/intention to complete cancer screening programmes or any measure of cancer symptom awareness/beliefs, knowledge of cancer risk factors, benefits of early detection and help seeking behaviour/confidence to detect a change that might be cancer. There had to be an explicit link between the targeted outcome and cancer.

(5) Studies: RCTs and cluster RCTs were included. Only randomized trials with at least one comparator arm were included in this review because these study designs provide the strongest evidence for evaluating effectiveness of interventions. ${ }^{29}$ 
1 All studies identified during the database search were assessed for relevance in a two-stage

2 screening process; firstly the studies were assessed based on the information contained in the title

3 and abstract. If at this stage the study appeared to meet the inclusion criteria, or if this was

4 unclear, the full article was retrieved. Papers selected for retrieval were primarily assessed by

5 one reviewer (SB) to determine inclusion given the clearly defined a-priori criteria. In ambiguous

6 cases, additional reviewers were consulted (LM, JY, DF).

7 2.4. Data extraction

8 Detailed information on sample demographics, $\mathrm{E} \underline{\mathrm{CB}} \mathrm{HW}$ characteristics (including

9 selection/training processes) and intervention characteristics was extracted and an example

10 extraction form is available in Appendix S2. Relevant information was also extracted to code

11 theory use, assess BCT prevalence and judge risk of bias, coded by one reviewer (SB). Five

12 articles were double coded by two reviewers (SB, LM) to establish agreement. During the double

13 coding process, advice was sought from a third reviewer (DF) regarding definitions of

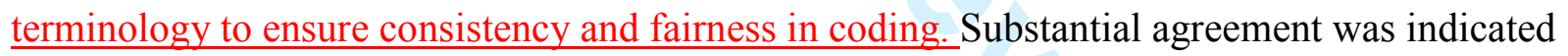
for theory coding (chance-corrected $\kappa=.72$ ) and risk of bias judgements (chance-corrected $\kappa=.90$ )

16 and moderate agreement for BCTs (chance-corrected $\kappa=.0 .55)$. However it is not recommended to use the Kappa statistic when coding the BCT taxonomy due to the high likelihood of BCTs $\underline{\text { being absent artificially lowering observed Kappa statistics. }}{ }^{30}$ Given this, reporting percentage agreement is more appropriate; this was almost perfect at $97 \%$. it was agreed that the majority reflect which was high at $97 \%$. Due to high agreement, one reviewer (SB) proceeded to code 
1

2

3

4

5 included articles. The framework consists of 33 questions which are rated "yes", "no", "don't

6 know" or "n/a". It has previously been used in systematic reviews to code theory use based on

7 intervention reports. ${ }^{32}$ The Theory Coding Scheme assesses the extent to which intervention

8 techniques are explicitly linked to theoretical constructs. A theoretical construct is defined as a

9 concept within a theory which predicts behaviour and is amenable to change e.g. perceived

10 susceptibility in the Health Belief Model. ${ }^{33}$ Percentages were calculated to assess the number of

11 studies scoring "yes" to each question. An overall theory score (range 0-8) was calculated using

12 the answers to items 3 to $11^{32}$

Behaviour change technique use. A hierarchical taxonomy of 93 BCTs was used to code the content of the interventions. ${ }^{26}$ The most recent hierarchical list was used, with published definitions guiding coding for each technique. The lead coder (SB) completed the online training resource for the BCT taxonomy. A BCT was not coded as present unless the text explicitly linked the technique to both the named target behaviour and the named population. For example, "ECBHWs helped participants identify their barriers to cancer screening and generated possible solutions to help overcome the barriers" adequately describes the problem solving BCT whereas stating that "barriers to cancer screening were discussed" is insufficient. Percentages were calculated to assess the proportion of studies using each BCT. 
Risk of bias. Using the Cochrane Collaboration's tool for assessing risk of bias ${ }^{34}$, a

2 judgement of low, unclear or high risk of bias was made for items related to randomization and

3 blinding procedures, incomplete data assessment, selective reporting and any other sources of

4 bias. The additional guidance provided by the Cochrane Collaboration was followed when

$5 \quad$ deciding between low, unclear or high risk judgements.

$7 \quad$ 2.5. Statistical analyses

8 Meta-analyses of screening attendance measures were performed in Comprehensive Meta-

9 Analysis 2.0. ${ }^{35}$ To account for the expected heterogeneity between studies, a random-effects

10 model was applied. ${ }^{36}$ First, 'adherence to cancer screening' was calculated as a pooled odds ratio

11 (O.R.) comparing the total numbers of participants who had taken part in screening for breast

12 cancer, colorectal cancer or cervical cancer following $\mathbb{L} \underline{\mathrm{CB} H W}$ interventions versus control conditions. Breast self-examination (BSE) and/or clinical breast examination (CBE) adherence

14 data were not classified as forms of cancer screening in these analyses. Data from both medical

15 records and self-report measures were used for analysis. Where studies had reported this

16 separately, a mean O.R. was calculated for medical record and self-report data and used in the

17 analysis. Similarly, where studies which had reported multiple cancer screening outcomes, a

18 mean O.R. was calculated from all eligible measures within each respective study.

19 The secondary outcome was 'Uptake of cancer screening amongst non-adherent samples'. 'Non-

20 adherent" refers to individuals, who at the time of participation in the study were non-compliant

21 with age appropriate recommended schedules of cancer screening. Thus "uptake" refers to the

22 total number of non-adherent individuals who attended cancer screening after receiving a 
ECBHW intervention (or control condition). Data from those studies which included only non-

2 adherent samples from the outset, and those studies which reported screening uptake among non-

3 adherent sub-groups of standard samples were used.

4 Heterogeneity between studies was quantified as Cochran's Q (with respective p-value) and I ${ }^{2}$

5 values. ${ }^{37}$ Funnel-plots were generated to assess the possibility of publication bias influencing the

6 main analyses and Egger's test ${ }^{38}$ and Begg \& Mazumdar's test ${ }^{39}$ were applied to quantify the risk

7 of publication bias. Where either was significant, a trim-and-fill analysis was applied to

8 recalculate the O.R. effect of $\mathrm{ECBHW}$ interventions after removing all studies which could

9 potentially be introducing publication bias. ${ }^{40}$

10 Sensitivity analyses examined the size of main effects (i) only among trials with full outcome

11 data or intention-to-treat analyses, and (ii) when using only medical record data or self-report

12 measures alone.

13 Subgroup analyses were used to investigate four potential moderators of overall intervention

14 effect. Firstly, the extent to which effects of $\mathrm{ECBHW}$ interventions on attendance were found

15 for specific cancer types was examined, specifically breast cancer (mammography), colorectal

16 cancer (any measure due to small number of included studies), or cervical cancer (Pap test),

17 individually. As the BSE and CBE data still related to an outcome of interest (early detection of

18 cancer symptoms), it was also included in this analysis. Secondly, moderator analyses considered

19 which settings may be most effective for delivering $\mathrm{ECBHW}$ interventions to improve cancer

20 screening adherence; comparing pooled effect sizes of interventions delivered in the home,

21 community, primary care settings-and those delivered by telephone only. A third subgroup

22 analysis explored modes of delivery to assess which may be most effective to improve cancer 
1 screening adherence; comparing pooled effect sizes of interventions delivered individually, in a

2 group format and those delivered using a combination approach (individual and group). ${ }^{41}$

3 Finally, a mixed-effects meta-regression was performed to explore the relationship between two

4 continuous moderators (number of BCTs applied and Theory Coding Scheme score) and

5 intervention effect size.

6

7 3. Results

$8 \quad 3.1$. Search results

9 The searches identified 2493 results; 123 full text versions were retrieved and assessed, of which

$10 \quad 3 \underline{3} 5$ articles were eligible for inclusion (screening process in Figure 1).

11 [Figure 1 insert here]

\section{3.2. Included studies and participant details}

13 Twenty individually randomized controlled trials and $1 \underline{3} \underline{5}$ cluster RCTs were included; $3 \underline{0} \underline{z}$ were 14 conducted in the USA, two in a US-Mexico border region and one was conducted in Belgium.

15 The majority of studies focused on female participants from ethnic minorities, with only nine ten 16 of the eligible studies including participants who were male (mean $=31.34 \%$, range $16.4-50.0)$.

17 The mean age of participants was more than 50 years in $1 \underline{78}$ studies, $1 \underline{01}$ studies had a mean age 18 less than 50 years, and six did not specify the average sample age. The average length of follow19 up across all studies was _ix eight months, ranging from immediately post-intervention to 24 20 months. For more key study characteristics see Appendix S3.

\section{3.2.1. Selection and training processes}


A selection process for the $\mathrm{ECBHWs}$ was described in $\mathrm{k}=7(2 \underline{1} \theta \%)$ studies $^{42-48}$, which included assessment of desirable skills/experience ${ }^{44-46}$, personality characteristics ${ }^{42,43,45,48}$ and

One study included personal experience of breast or cervical cancer as a selection criterion for

\section{LCBHWs. ${ }^{51}$} intervention period, practice sessions and a written examination.. ${ }^{23,24,42,44,45,47,49,52-55,59,60,62-66}$

13 A form of research skills training including the principle of randomization and the importance of

21 A theory base was explicitly reported (i.e., coded yes to item 5 of Theory Coding Scheme) in

$22 \mathrm{k}=1 \underline{57}(4 \underline{5} 9 \%)$ interventions. ${ }^{23,25,43-45,53-55,59,60,62-64,67,68}$ Of these $1 \underline{57}$ studies, a combination of

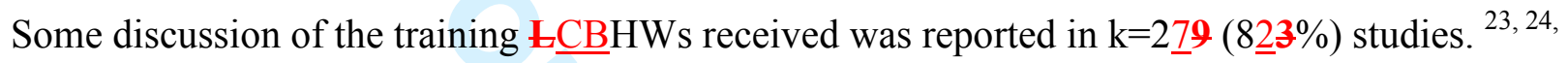
42-60, 62-67 The most commonly used pedagogic methodologies for training LCB aids/flip charts, practice intervention sessions, and role-playing. A competency assessment measure was described in $\mathrm{k}=1 \underline{8} 9$ (5둥) studies including; ongoing supervision throughout protocol adherence was reported in $\mathrm{k}=12$ (3ㅜ4\%) studies. ${ }^{24,42,44,45,49,50,52,53,55,62-64}$ The duration of training was described in $\mathrm{k}=1 \underline{78}(5 \underline{2} 1 \%)$ studies and the average duration was 11 hours (range: $4-24$ hours). ${ }^{24,42,44,47-49,51-55,59,60,62,64-66}$ For the majority of studies, training was 
1 theories was reported in $\mathrm{k}=\underline{8} 9(53 \%)$ (range 2-4). ${ }^{23,25,43,45,54,60,63,64}$ The Transtheoretical Model was applied in $\mathrm{k}=\underline{8} \mathbf{1 0}(5 \underline{3} 9 \%)$ studies and the Health Belief Model was reported in $\mathrm{k}=\underline{7} 8(47 \%)$ studies. At least one explicit link between an intervention technique and a theory-relevant

4 construct was reported in $\mathrm{k}=\underline{6} 7(4 \underline{01} \%)$ interventions. ${ }^{23,25,60,62,64,68}$ At least one explicit link between a theory-relevant construct and an intervention technique was reported in $\mathrm{k}=\underline{9} \mathbf{1 0}$ (6059\%) interventions. ${ }^{23,54,55,60,62-64,67,68}$ Theoretically relevant constructs (self-efficacy and perceived severity) were measured pre and post-intervention in $\mathrm{k}=\underline{2} \mathbf{3}(\underline{6} 9 \%)$ studies. ${ }^{44,55}$ Only 8 one study discussed the results in relation to the theoretical basis of the intervention. ${ }^{55}$ Overall, 9 the Theory Coding Scheme illustrated poor theory application; of the $3 \underline{3} 5$ interventions, $\mathrm{k}=18$ $(5 \underline{51} \%)$ scored zero and the highest score was four out of a possible eight. This pattern of results 11 precluded assessment of theory score as a moderator variable.

\section{3.2.3. Behaviour change technique use}

13 Overall, 21 of 93 possible BCTs were present in the interventions included in this review.

14 Interventions incorporated between zero and nine BCTs with a mean of four BCTs in each

15 intervention. Table 1 demonstrates that the most commonly observed techniques involved

16 providing practical social support, prompts and cues and problem solving. This remained the

17 case when results were limited to the BCTs delivered by the $\mathrm{ECBHW}$ only and other

18 components such as videos and media campaigns were discounted.

\section{9 [Table 1 insert here]}

\section{3.2.4. Risk of bias}

21

22 Overall, study quality was poor (see Appendix S4). Only one study had a published protocol. ${ }^{67}$, 
$1{ }^{69}$ Therefore the risk of bias for selective reporting was coded high for all remaining studies.

2 Other areas of common bias were reliance on self-reported outcomes, inadequate blinding of key

3 personnel and attrition bias. Attrition rates larger than $25 \%$ were reported in $\mathrm{k}=10(\underline{3029} \%)$

4 studies. $^{43,44,46,51,55,58,65,68,70,71}$

5 3.3. Effects of lay health worker interventions on cancer screening adherence

6 Results of all meta-analyses are displayed in Table 2. Adherence screening data for the primary

7 outcome was available from a total of 21,990 participants across 30 studies; 11,487 were

8 assigned to $\mathrm{E} \underline{\mathrm{CB}} \mathrm{HW}$ interventions, 10,503 to control conditions. ${ }^{23-25,42-60,62-68,72}$ Random-effects

9 meta-analyses found that $\mathrm{ECBHW}$ interventions resulted in significantly greater uptake of

10 overall cancer screening (see Figure 2 ) than control conditions (O.R. $=1.90,95 \% \mathrm{CI}=1.60-2.26$,

$11 \mathrm{p}<0.001)$, although there was considerable heterogeneity among study findings $(\mathrm{Q}=199.9$,

$\left.12 \mathrm{p}<0.01, \mathrm{I}^{2}=85.5 \%\right)$.

13 [Figure 2 insert here]

$14 \quad 3.4$. Publication bias and sensitivity analyses

15 Although the risk of publication bias was not statistically significant according to Begg \&

16 Mazumdar's test (Kendall's t=0.15, $\mathrm{p}=0.24)$, Egger's test did indicate publication bias $(\mathrm{p}=0.007)$.

17 Nonetheless, the Duval and Tweedies trim-and-fill analysis showed that, after removing 12

18 studies which may introduce publication bias, $\mathrm{E} \underline{\mathrm{CB} H W}$ interventions still significantly improved 19 screening adherence more than control conditions (O.R. $=1.40,95 \%$ C.I. $=1.15-1.69)$.

20 A sensitivity analysis was performed on the 19 studies which used ITT analyses or complete

21 outcome data, with 16,300 participants in total. ${ }^{23,42,45-47,49-51,53,54,56-58,62,63,65-67,72}$ This found 
1 that $\mathrm{E} \underline{\mathrm{CBHW}}$ interventions also resulted in increased cancer screening adherence on an intention-to-treat basis $(\mathrm{O} . \mathrm{R} .=1.98,95 \% \mathrm{CI}=1.57-2.49, \mathrm{p}<0.001)$ again with significant

3 heterogeneity between studies $\left(\mathrm{Q}=146.2, \mathrm{p}<0.01, \mathrm{I}^{2}=87.7 \%\right)$.

4 Further sensitivity analyses showed that $\mathrm{ECBHW}$ interventions resulted in significantly greater 5

$656,58,66,72(\mathrm{~N}=8, \mathrm{n}=11658$, O.R. $=2.092,95 \% \mathrm{CI}=1.36-3.21, \mathrm{p}=0.001)$ or self-report data ${ }^{24,25,42,43,}$

$746,48,51,52,55-60,62-64,68(\mathrm{~N}=18, \mathrm{n}=8620$, O.R. $=1.686,95 \% \mathrm{CI}=1.40-2.03, \mathrm{p}<0.001)$.

8 3.5. Effects of lay health worker interventions on cancer screening uptake in non-adherent 9 samples We also examined the effect of $\mathrm{E} \underline{\mathrm{CBHW}}$ interventions on uptake of any cancer screening among 11 non-adherent samples (see Appendix S5). Across 20 studies with 12,769 previously non12 adherent individuals, LHW interventions resulted in significantly greater likelihood of receiving 13 cancer screening at follow-up compared to control conditions $23,24,42,44-49,51,53-55,57,58,65-68,72$

14 (O.R. $=2.40,95 \%$ C.I. $=1.85-3.11, \mathrm{p}<0.001)$. However, there was statistical evidence of both 15 between-study heterogeneity $\left(\mathrm{Q}=149.1, \mathrm{p}<0.01, \mathrm{I}^{2}=87.3 \%\right)$ and publication bias (Egger's $\mathrm{t}=2.33$, $16 \mathrm{p}=0.03)$. Nonetheless, the results still significantly favoured LHW interventions after applying 17 the trim-and-fill analysis $(\mathrm{N}=9$, O.R. $=1.58,95 \%$ C.I. $=1.19-2.09)$.

18 3.6. Factors associated with intervention efficacy

19 Further analyses were performed to examine effects of $\underline{\mathrm{E}} \underline{\mathrm{CB}} \mathrm{HW}$ interventions on the primary 20 outcome (i.e. adherence to cancer screening) when (i) focusing on specific cancer screening 
1 types, (ii) comparing different settings of delivery, (iii) comparing different modes of delivery,

2 and (iv) number of BCTs used in interventions (see Table 2).

\section{3 [Table 2 insert here]}

4

5

6

8

9

10

11

12

13

14

15

16

17

18

19

20

21
Significant benefits of $\mathrm{E} \underline{\mathrm{CBHW}}$ interventions were observed across each type of screening measure, including mammography $(\mathrm{k}=13, \mathrm{n}=9641, \mathrm{O} . \mathrm{R} .=1.923,95 \% \mathrm{CI}=1.44-2.57, \mathrm{p}<0.001)$, Pap testing $(\mathrm{k}=15, \mathrm{n}=5276$, O.R. $=1.922,95 \% \mathrm{CI}=1.39-2.64, \mathrm{p}<0.001)$ and colorectal cancer screening $(\mathrm{k}=7, \mathrm{n}=9425$, O.R. $=1.983,95 \% \mathrm{CI}=1.31-3.01, \mathrm{p}<0.001)$. Furthermore, the benefits of ECBHW interventions were demonstrated for both BSE $(\mathrm{k}=4, \mathrm{n}=1493$, O.R. $=1.554,95 \%$ $\mathrm{CI}=1.17-2.07, \mathrm{p}=0.003)$ and $\mathrm{CBE}(\mathrm{N}=5, \mathrm{n}=2582, \mathrm{O} . \mathrm{R} .=1.528,95 \% \mathrm{CI}=1.19-2.00, \mathrm{p}=0.001)$.

Twenty-six studies used home or community settings, and found $\mathrm{L} \underline{\mathrm{CB} H W}$ interventions were effective when delivered in the home $(\mathrm{k}=13, \mathrm{n}=5779, \mathrm{O} . \mathrm{R} .=1.808,95 \% \mathrm{CI}=1.40-2.34, \mathrm{p}<0.001)$

or the community $(\mathrm{k}=13, \mathrm{n}=5621, \mathrm{O} . \mathrm{R} .=2.249,95 \% \mathrm{CI}=1.63-3.11, \mathrm{p}<0.001)$. Four studies examined Only two-studies each-examined primary care or telephone-only interventions, and

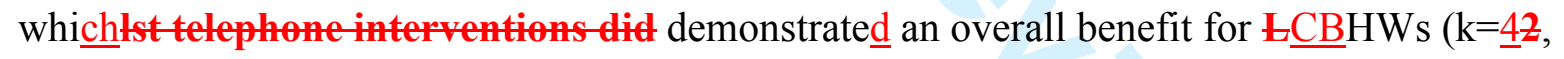
$\mathrm{n}=\underline{105904402}, \mathrm{O} . \mathrm{R} .=1.3 \underline{2219}, 95 \% \mathrm{CI}=1.14-1.5 \underline{43}, \mathrm{p}<0.001$ ), primary care interventions did $\operatorname{not}(k=2, n=6188,0 . R .=1.396,95 \%, C I=0.96-1.97, p=0.085)$

Regarding mode of delivery, $\mathrm{E} \underline{\mathrm{CB}} \mathrm{HW}$ interventions were equally as effective when delivered individually $(\mathrm{k}=16, \mathrm{n}=15256, \mathrm{O} . \mathrm{R} .=1.680,95 \% \mathrm{CI}=1.40-2.02, \mathrm{p}<0.001)$ or in a group format $(\mathrm{k}=5, \mathrm{n}=2378$, O.R. $=1.633,95 \% \mathrm{CI}=1.36-1.96, \mathrm{p}<0.001$. A combined delivery approach (individual and group elements present) was found to be most effective $(k=8, n=3636$, O.R. $=2.463,95 \% \mathrm{CI}=1.43-4.23, \mathrm{p}=0.001)$ 
1 Mixed-effects meta-regression found that number of BCTs used in $\mathrm{ECBHW}$ interventions held a

2 trend-level association with observed effect size (see Appendix S6); as the O.R. for cancer

3 screening adherence from $\mathrm{E} \underline{\mathrm{CB}} \mathrm{HW}$ interventions increased with the number of BCTs used (B

$4=0.061, \mathrm{~S} . \mathrm{E} .=0.036, \mathrm{Z}=1.71, \mathrm{p}=0.088)$. A post-hoc subgroup analysis was conducted to

5 examine the effects of $\underline{\mathrm{GB} H W}$ interventions which used a high number of BCTs (four or more)

6 vs. those which used a low number of BCTs (three or less) (Table 2; see Appendix S7). These

7 cut offs were selected in light of the average intervention containing four BCTs. ELBHW

8 interventions with a high number of BCTs increased cancer screening with an odds ratio of 2.27

$9 \quad(\mathrm{k}=13, \mathrm{n}=6557,95 \%$ C.I. $=1.78-2.89, \mathrm{p}<0.001)$, whereas those with low use of BCTs increased

10 cancer screening by only O.R. $=1.66(\mathrm{k}=17, \mathrm{n}=15433,95 \%$ C.I. $=1.34-2.05, \mathrm{p}<0.001)$, although

11 the difference between High and Low BCT subgroups fell short of statistical significance

$12(\mathrm{Q}=3.613, \mathrm{p}=0.057)$.

13 The finding of poor theory application precluded assessment of theory score as a moderator

14 variable.

3.7. Effects of lay healh worker interventions on cancer preventative behaviours

Two-studies examined the effect of LHW interventions on promoting cancer preventative

significant improvements in self-reported daily consumption of fruits and vegetables 
1

2

involving LHWs resulted in-significantly greater recreational exercise or increased fruit and vegetable consumption at follow up when-compared to the control group.

\section{4. Discussion}

\section{4.1. Summary of main findings}

6 The results from this systematic review and meta-analysis indicate that $\mathrm{E} \underline{\mathrm{CBHW}}$ interventions

7 are associated with a statistically significant increase in cancer screening attendance, with a

8 stronger effect size observed for participants previously non-adherent with recommended

9 schedules of cancer screening. Similar odds ratios were found for screening for breast, cervical

10 and colorectal cancer. The overall pattern of results was robust to sensitivity analyses, with

11 similar effect sizes found irrespective of source of outcome measure (self-report or medical

12 records), or whether intention-to-treat analyses were conducted or not. There was little use of

13 theory in these interventions, making it difficult to identify the means by which the interventions

14 changed screening attendance. The interventions generally did not use many BCTs, in line with

15 the apparent lack of use of theory to develop interventions. Methodological quality of included 16 studies was generally poor.

17

18 4.2. Strengths \& limitations

19

20 The findings of this review build upon the results of previous smaller reviews examining

21 mammography screening only. ${ }^{11,22}$ This review has been the first to illustrate the effectiveness

22 of $\underline{\mathrm{ECBHW}}$ interventions across all types of cancer screening and other measures of early 
1 detection (BSE and CBE). This allows the present review to draw conclusions relating to the

2 efficacy of $\mathrm{ECBHW}$ interventions across screening for breast, cervical and colorectal cancer. Further the present review has been the first to apply the BCT taxonomy and Theory Coding Scheme and a rigorous risk of bias tool to cancer specific $\mathrm{E}$ CBHW interventions.

6 A limitation of the present review was that, as many terms are used to describe $\underline{\mathrm{LB}} \underline{\mathrm{BW}} \mathrm{H}$, it may

7 be possible that we did not extract all relevant articles in the existing literature. However, to

8 avoid this, a systematic, comprehensive electronic search was conducted using 45 different terms

9 for $\mathrm{ECBHW}$ after a consultation with a trained health science librarian. The present review

10 identified many more studies than did previous systematic reviews, and included studies

11 identified by these previous reviews, indicating that this was not a major problem.

13 There were however, limitations of the primary evidence base that the present review has

14 identified, but which limit the conclusions that can be drawn. First, substantial heterogeneity

was found across the analyses. This between-study heterogeneity is unsurprising, given the clear differences between CBHW interventions in terms of the intervention components used, cancers $\underline{\text { screened for, guidelines in place at screening, populations studied and outcome measures }}$ applied, all sources of heterogeneity which are typical to meta-analytic research. Although this heterogeneity was statistically accounted for by the random-effects model applied, it is acknowledged that the strength of the conclusions from this review are limited as a result.

21 Second,First, the research base itself was limited to cancer screening outcomes and

22 heterogeneity prevented the combination of secondary outcome data into meaningful categories

23 to examine effectiveness of $\underline{\mathrm{LCBH}} \underline{\mathrm{HW}}$ interventions in other areas of early cancer $\underline{\text { diagnosis }}$ 
1 awareness (e.g., knowledge, intention, self-efficacy). It was also notable that all but one of the

2 included studies were implemented in the USA and therefore caution is needed to generalize and

3 apply findings to countries with different healthcare systems, cancer screening guidelines and

4 ethnic compositions.

5

6 The review identified several methodological biases present in the primary studies; high rates of

7 attrition, reliance on self-reported outcomes, and failure to report blinding which could have

8 contributed to biased estimates of interventional efficacy. However it is important to note that $\underline{\text { there is an inherent difficulty with blinding in trials assessing the effectiveness of CBHWs. In the }}$ $\underline{\text { majority of cases CBHWs recruit participants from their own social networks/communities as }}$ shared characteristics between the CBHW and the audience facilitate learning and increase the with one of the presumed mechanisms of effectiveness. Nevertheless, sensitivity analyses suggested that these methodological limitations did not unduly affect the overall conclusions.

15 Furthermore, publication bias was indicated but after removing studies with smaller sample 16 sizes, improvements in screening attendance in $\underline{\mathrm{EBHWW}}$ conditions remained significant.

\subsection{Interpretation of main findings}

This study has been the first to illustrate the effectiveness of $\underline{\mathrm{LCBHW}}$ interventions beyond 

1 reviews have suggested. ${ }^{11,22}$ Subgroup analyses revealed similar effects for breast, cervical and $\underline{\text { colorectal cancer screening. }}$

4 The review provides an updated description of $\mathrm{ECBHW}$ training characteristics, crucial to the reporting of selection and training processes in $\mathrm{E} \underline{\mathrm{CBHW}}$ literature. A conceptual model has been

7 proposed to standardize the reporting of such processes in the future. ${ }^{75}$ This transparency will

8 facilitate learning for future intervention development. However, how training content is selected

9 remains unclear, and the lack of readily reported information about components of training

10 hinders the development of evidence based training packages.

12 The present review explored potential moderators of effectiveness to provide practical

13 recommendations for future $\mathrm{E} \underline{\mathrm{CB}} \mathrm{HW}$ intervention development. Moderator analyses demonstrated that $\mathrm{E} \underline{\mathrm{CBHW}}$ interventions were equally as effective across all types of cancer screening. Subgroup analyses revealed that interventions were effective when delivered in community and home settings, over the telephone and when using both individual and group modes of delivery were used. Only one of the included interventions was delivered in a primary care setting. ${ }^{70}$ However as it measured knowledge changes only, there was no eligible data to include in the meta-analysis of effects on screening behaviour. No beneficial effect of the CBHW was found when compared to usual care but The eurrent evidence did not support the effectiveness of the LHW approach in primary care settings. However, only two-studies used this type of setting and so additional research is needed to establish whether delivering a LCBHW intervention in primary care settings has the potential to be effective. 
2 An in-depth analysis with Theory Coding Scheme items ${ }^{31}$ revealed substantial evidence of

3 'theory inspired' interventions, whereby theory is not used to identify methods to change

4 behaviour, as evidenced by poor linkage between BCTs and theoretical constructs. ${ }^{76}$ This

5 absence of an explicit theoretical basis for interventions makes it difficult to identify why

6 interventions worked, and how future interventions could work better: it prevents an

7 accumulation of knowledge on what basis future $\underline{\mathrm{ECB}} \underline{\mathrm{HW}}$ interventions should be

8 developed. ${ }^{77},{ }^{78}$ Few BCTs were generally used: $\underline{\mathrm{LB}} \underline{\mathrm{BHW}}$ were found to most frequently provide 9 practical support to participants, resolve barriers and prompt the desired behaviour. There was a

10 trend $(\mathrm{p}=0.057)$ towards the use of BCTs being positively associated with intervention

11 effectiveness; larger effect sizes were observed in studies using more than four BCTs $(\mathrm{OR}=$

12 2.27) compared to those using three or fewer $(\mathrm{OR}=1.66)$. Given that few BCTs were generally

13 used, this would have reduced the association between BCTs and effectiveness, due to a lack of

14 range. Given this, at the least the inclusion of more BCTs should be considered in future studies,

15 to examine whether this results in increased intervention effectiveness.

Of note was the exclusive emphasis on cancer screening behaviour observed in this review. Half

of the included studies measured secondary outcomes related to knowledge but this was limited

$\underline{\text { to awareness of screening procedures, the benefits of early diagnosis, and cancer risk factors. }}$

International comparisons have reported greater endorsement of barriers to help-seeking in UK

$\underline{\text { adults }}^{79}$ in particular regarding the perception of not wanting to waste the doctor's time or

cause a fuss. ${ }^{80}$ Despite public awareness campaigns increasing cancer symptom awareness, GP 
engagement is more pronounced in lower socioeconomic groups. ${ }^{81}$ This suggests that broadening the CBHW remit to increasing symptom recognition and promotion of help-seeking behaviour is a worthwhile avenue to pursue, particularly in a UK context where barriers to helpseeking remain high. review support the implementation of $\underline{\mathrm{E}} \underline{\mathrm{CBHW}}$ interventions to increase cancer screening in

Mixed results of effectiveness of LHW approaches were observed in the two included studies promoting cancer preventative behaviours. It is likely the effectiveness was adversely affected by implementation challenges; in one study, only $10 \%$ of people in the LHW only and combined groups reported having talked with a LHW. ${ }^{61}$ The lack of eligible explicit cancer risk-related lifestyle interventions delivered by LHWs is reflective of lifestyle interventions focusing predominantly on chronic diseases including cardiovascular diseases. ${ }^{75}$ There is evidence that UK people were less likely to identify unhealthy diet, excessive alcohol consumption or physical inactivity as risk factors for cancer compared to heart disease ${ }^{76}$, indicating a need for awareness interventions that may encourage risk reducing behaviour change. ${ }^{77}$

\subsection{Implications and future research directions}

This review has demonstrated that $\mathrm{E} \underline{\mathrm{CB}} \mathrm{HW}$ interventions are a promising approach to addressing cancer screening disparities in the US, particularly in ethnic minority groups.

Notwithstanding limitations in evidence base, the evidence and size of effect obtained in this ethnic minorities. Based on the current evidence base, those previously non-adherent with recommended schedules of cancer screening are most likely to benefit. 
1 However substantial work is still needed to fully elucidate the conditions key to intervention

2 effectiveness using methodologically sound studies in different populations. As such, the

3 following recommendations for future research are made:

4

5 a) Guidelines such as the Template for intervention description and replication (TIDieR) ${ }^{82}$ should be used more to increase transparency and facilitate learning between researchers. This would contribute to the identification of key mechanisms of behaviour change, the most effective components of training packages and reduce heterogeneity prevalent across studies.

b) Use of explicit theory in the development and evaluation of interventions, to target hypothetical mediators of behaviour change, such as knowledge and intent, to increase intervention effectiveness. The use of theory in evaluation of such interventions should clarify the processes of change initiated by $\mathrm{LCBHWs}_{\text {C }}$ This would help inform the need for refinement in terms of intervention components and BCTs.

c) The use of a greater range of BCTs should be considered in future interventions, to allow an assessment of whether this would result in greater intervention effectiveness, compared to $\mathrm{E} \underline{\mathrm{CB}} \mathrm{HW}$ interventions conducted to date.

d) Further exploring the influence demographic factors such as participant gender has on $\underline{\mathbf{E} B H W}$ intervention effectiveness across all cancer screening programmes. There is a clear need for more research using populations outside the US to assess whether such interventions are more or less effective in different settings. 
1 e) Exploratory Further work should needs to be conducted to assess the feasibility of using CBHWs to increase symptom awareness and promote help-seeking behaviour. improve awareness of cancer related lifestyle factors and to establish the effectiveness of HHWs in this area as well as other areas of early diagnosis such as help-seeking behaviour.

\section{5. Conclusions}

8

In conclusion, this review has found that $\mathrm{E}$ CBHWs are an effective resource for increasing cancer screening uptake, particularly for those previously non-adherent with recommended schedules of cancer screening. It is yet unknown whether $\mathrm{E}$ CBHW interventions are an effective approach for $\underline{t}$ other areas of early diagnosis including symptom awareness and help-seeking behaviour. reduce lifestyle related cancer risk factors. The number of explicitly described BCTs used was found to be positively associated with effect size, although this did not reach

14 statistical significance. At present, these conclusions are almost entirely limited to the US

15 healthcare context and ethnic composition and limited by poor methodological quality and

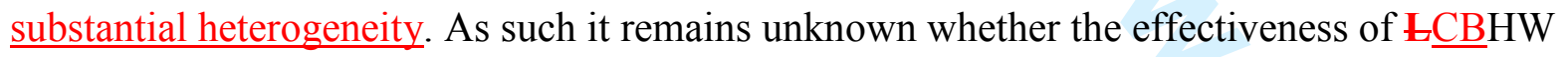

17 interventions would translate to different countries or other areas pertinent to early cancer

18 diagnosis such as help-seeking behaviour.

\section{Conflicts of interest}

21 The authors declare no potential conflicts of interest. 


\section{References}

References marked with an asterisk indicate studies included in the systematic review

1. World Health Organization. Global action plan for the prevention and control of NCDs 20132020 [Internet]. World Health Organization; 2013. Available from: http://apps.who.int/iris/bitstream/10665/94384/1/9789241506236 eng.pdf?ua=1

2. World Health Organization. Guide to cancer early diagnosis [Internet]. World Health Organization; 2017. Available from:

http://apps.who.int/iris/bitstream/10665/254500/1/9789241511940-eng.pdf?ua=1

3. Ott JJ, Ullrich A, Miller AB. The importance of early symptom recognition in the context of early detection and cancer survival. Eur J Cancer. 2009;45:2743-8

4. CRUK. Cancer Awareness Measure (CAM) Key Findings Report; 2014 \& Trends Analysis (2008-2014) [Internet]. 2014. Available from: http://www.cancerresearchuk.org/sites/default/files/cam key findings report 2014 trends analysis_v5.pdf

$\overline{5}$. Health and Social Care Information Centre. Cervical Screening Programme - England, 201415 [Internet]. National Statistics; 2015. Available from: http://content.digital.nhs.uk/catalogue/PUB18932/nhs-cervical-stat-eng-2014-15-rep.pdf 6. Health and Social Care Information Centre. Breast Screening Programme - England, 2014-15 [Internet]. National Statistics; 2016. Available from: http://content.digital.nhs.uk/catalogue/PUB20018/bres-scre-prog-eng-2014-15-rep.pdf 7. Smith RA, Manassaram-Baptiste D, Brooks D, Doroshenk M, Fedewa S, Saslow D, et al. Cancer screening in the United States, 2015: A review of current American Cancer Society guidelines and current issues in cancer screening. CA Cancer J Clin. 2015;65:30-54

8. Power E, Wardle J. Change in public awareness of symptoms and perceived barriers to seeing a doctor following Be Clear on Cancer campaigns in England. Br J Cancer. 2015;112:S22-S6 9. Guessous I, Dash C, Lapin P, Doroshenk M, Smith RA, Klabunde CN. Colorectal cancer screening barriers and facilitators in older persons. Prev Med. 2010;50:3-10 10. Smith LK, Pope C, Botha JL. Patients' help-seeking experiences and delay in cancer presentation: a qualitative synthesis. The Lancet. 2005;366:825-31 11. Lewin SA, Dick J, Pond P, Zwarenstein M, Aja G, van Wyk B, et al. Lay health workers in primary and community health care. The Cochrane database of systematic reviews. 2005. CD004015.

12. Witmer A, Seifer SD, Finocchio L, Leslie J, O'Neil EH. Community health workers: integral members of the health care work force. Am J Public Health. 1995;85:1055-8 13. Hou S-I, Roberson K. A Systematic Review on US-Based Community Health Navigator (CHN) Interventions for Cancer Screening Promotion - Comparing Community- Versus ClinicBased Navigator Models. J Cancer Educ. 2015;30:173-86 14. Rhodes SD, Foley KL, Zometa CS, Bloom FR. Lay Health Advisor Interventions Among Hispanics/Latinos: A Qualitative Systematic Review. Am J Prev Med. 2007;33:418-27 15. Freeman HP. A model patient navigation program. Oncology Issues. 2004;19:44-6 16. Wells KJ, Battaglia TA, Dudley DJ, Garcia R, Greene A, Calhoun E, et al. Patient navigation: State of the art or is it science? Cancer. 2008;113:1999-2010 17. Gibbons MC, Tyus NC. Systematic review of U.S.-based randomized controlled trials using community health workers. Prog Community Health Partnersh. 2007;1:371-81 
18. Islam NS, Zanowiak JM, Riley L, Nadkarni SK, Kwon SC, Trinh-Shevrin C. Characteristics of Asian American, Native Hawaiian, and Pacific Islander community health worker programs: a systematic review. J Health Care Poor Underserved. 2015;26:238-68 19. Viswanathan M, Kraschnewski JL, Nishikawa B, Morgan LC, Honeycutt AA, Thieda P, et al. Outcomes and Costs of Community Health Worker Interventions: A Systematic Review. Med Care. 2010;48:792-808

20. Wadler BM, Judge CM, Prout M, Allen JD, Geller AC. Improving Breast Cancer Control via the Use of Community Health Workers in South Africa: A Critical Review. J Oncol.

2011;2011:H382-7

21. Kim K, Choi JS, Choi E, Nieman CL, Joo JH, Lin FR, et al. Effects of Community-Based Health Worker Interventions to Improve Chronic Disease Management and Care Among Vulnerable Populations: A Systematic Review. Am J Public Health. 2016;106:e3-e28 22. Wells KJ, Luque JS, Miladinovic B, Vargas N, Asvat Y, Roetzheim RG, et al. Do Community Health Worker Interventions Improve Rates of Screening Mammography in the United States? A Systematic Review. Cancer Epidemiol Biomarkers Prev. 2011;20:1580-98 23. *Byrd TL, Wilson KM, Smith JL, Coronado G, Vernon SW, Fernandez-Esquer ME, et al. AMIGAS: A multicity, multicomponent cervical cancer prevention trial among Mexican American women. Cancer. 2013;119:1365-72

24. *Nguyen BH, Stewart SL, Nguyen TT, Bui-Tong N, McPhee SJ. Effectiveness of Lay Health Worker Outreach in Reducing Disparities in Colorectal Cancer Screening in Vietnamese Americans. Am J Public Health. 2015; 105:2083-9 25. *Paskett ED, McLaughlin JM, Lehman AM, Katz ML, Tatum CM, Oliveri JM. Evaluating the Efficacy of Lay Health Advisors for Increasing Risk-Appropriate Pap Test Screening: A Randomized Controlled Trial among Ohio Appalachian Women. Cancer Epidemiol Biomarkers Prev. 2011;20:835-43

26. Michie S, Richardson M, Johnston M, Abraham C, Francis J, Hardeman W, et al. The Behavior Change Technique Taxonomy (v1) of 93 Hierarchically Clustered Techniques: Building an International Consensus for the Reporting of Behavior Change Interventions. Ann Behav Med. 2013;46:81-95

27. French DP, Olander EK, Chisholm A, Mc Sharry J. Which Behaviour Change Techniques Are Most Effective at Increasing Older Adults' Self-Efficacy and Physical Activity Behaviour? A Systematic Review. Ann Behav Med. 2014;48:225-34

28. Moher D, Liberati A, Tetzlaff J, Altman DG, Group P. Preferred reporting items for systematic reviews and meta-analyses: the PRISMA statement. PLoS Med. 2009;6:e1000097 29. Evans D. Hierarchy of evidence: a framework for ranking evidence evaluating healthcare interventions. J Clin Nurs. 2003;12:77-84

30. Wood CE, Richardson M, Johnston M, Abraham C, Francis J, Hardeman W, et al. Applying the behaviour change technique (BCT) taxonomy v1: a study of coder training. Transl Behav Med. 2015;5:134-48

31. Michie S, Prestwich A. Are interventions theory-based? Development of a theory coding scheme. Health Psychol. 2010;29:1-8

42 32. Prestwich A, Sniehotta FF, Whittington C, Dombrowski SU, Rogers L, Michie S. Does

43 theory influence the effectiveness of health behavior interventions? Meta-analysis. Health

44 Psychol. 2014;33:465-74 
33. Janz NK, Becker MH. The Health Belief Model: A Decade Later. Health Educ Q. 1984;11:147

34. Higgins JPT, Altman DG, Gøtzsche PC, Jüni P, Moher D, Oxman AD, et al. The Cochrane Collaboration's tool for assessing risk of bias in randomised trials. BMJ. 2011;343:d5928 35. Borenstein M, Hedges L, Higgins J, Rothstein H. Comprehensive Meta Analysis. 2 ed. Englewood, NJ: Biostat; 2005.

36. DerSimonian R, Kacker R. Random-effects model for meta-analysis of clinical trials: An update. Contemp Clin Trials. 2007;28:105-14 37. Higgins JPT, Thompson SG. Quantifying heterogeneity in a meta-analysis. Stat Med. 2002;21:1539-58

38. Egger M, Smith GD, Schneider M, Minder C. Bias in meta-analysis detected by a simple, graphical test. $B M J$. 1997;315:629-34

39. Begg CB, Mazumdar M. Operating Characteristics of a Rank Correlation Test for Publication Bias. Biometrics. 1994;50:1088-101

40. Duval S, Tweedie R. Trim and Fill: A Simple Funnel-Plot-Based Method of Testing and Adjusting for Publication Bias in Meta-Analysis. Biometrics. 2000;56:455-63

41. Dombrowski SU, O'Carroll RE, Williams B. Form of delivery as a key 'active ingredient' in behaviour change interventions. Br J Health Psychol. 2016;21:733-40 42. *Lam TK, Mc Phee SJ, Mock J, Wong C, Doan HT, Nguyen T, et al. Encouraging Vietnamese-American Women to Obtain Pap Tests Through Lay Health Worker Outreach and Media Education. J Gen Intern Med. 2003; 18:516-24 43. *Navarro AM, Senn KL, McNicholas LJ, Kaplan RM, Roppé B, Campo MC. Por La Vida model intervention enhances use of cancer screening tests among Latinas. Am J Prev Med. 1998; $15: 32-41$ 44. *O'Brien MJ, Halbert CH, Bixby R, Pimentel S, Shea JA. Community Health Worker Intervention to Decrease Cervical Cancer Disparities in Hispanic Women. J Gen Intern Med. 2010;25:1186-92 45. *Paskett E, Tatum C, Rushing J, Michielutte R, Bell R, Foley KL, et al. Randomized Trial of an Intervention to Improve Mammography Utilization Among a Triracial Rural Population of Women. J Natl Cancer Inst. 2006;98:1226-37 46. *Sung JF, Blumenthal DS, Coates RJ, Williams JE, Alema-Mensah E, Liff JM. Effect of a cancer screening intervention conducted by lay health workers among inner-city women. $\mathrm{Am} \mathrm{J}$ Prev Med. 1997;13:51-7 47. *Han H-R, Song Y, Kim M, Hedlin HK, Kim K, Ben Lee H, et al. Breast and Cervical Cancer Screening Literacy Among Korean American Women: A Community Health WorkerLed Intervention. Am J Public Health. 2017;107:159-65 48. *Mock J, McPhee SJ, Nguyen T, Wong C, Doan H, Lai KQ, et al. Effective Lay Health Worker Outreach and Media-Based Education for Promoting Cervical Cancer Screening Among Vietnamese American Women. Am J Public Health. 2007;97:1693-700 49. *Goelen G, De Clercq G, Hanssens S. A community peer-volunteer telephone reminder call to increase breast cancer-screening attendance. Oncol Nurs Forum. 2010;37:E312-7 50. *Hunter JB, de Zapien JG, Papenfuss M, Fernandez ML, Meister J, Giuliano AR. The Impact of a Promotora on Increasing Routine Chronic Disease Prevention among Women Aged 40 and Older at the U.S.-Mexico Border. Health Educ Behav. 2004;31:18S-28S 
51. *Jandorf L, Bursac Z, Pulley L, Trevino M, Castillo A, Erwin DO. Breast and cervical cancer screening among Latinas attending culturally specific educational programs. Prog Community Health Partnersh. 2008;2:195-204 52. *Nguyen TT, Le G, Nguyen T, Le K, Lai K, Gildengorin G, et al. Breast Cancer Screening Among Vietnamese Americans: A Randomized Controlled Trial of Lay Health Worker Outreach. Am J Prev Med. 2009;37:306-13

53. *Nuño T, Martinez ME, Harris R, García F. A Promotora-administered group education intervention to promote breast and cervical cancer screening in a rural community along the U.S.-Mexico border: a randomized controlled trial. Cancer Causes Control. 2011;22:367-74 54. *Russell KM, Champion VL, Monahan PO, Millon-Underwood S, Zhao Q, Spacey N, et al. Randomized Trial of a Lay Health Advisor and Computer Intervention to Increase Mammography Screening in African American Women. Cancer Epidemiol Biomarkers Prev. 2010; $19: 201-10$ 55. *Sadler GR, Ko CM, Wu P, Alisangco J, Castañeda SF, Kelly C. A Cluster Randomized Controlled Trial to Increase Breast Cancer Screening Among African American Women: The Black Cosmetologists Promoting Health Program. J Natl Med Assoc. 2011;103:735-45 56. *Taylor VM, Carey Jackson J, Yasui Y, Kuniyuki A, Acorda E, Marchand A, et al. Evaluation of an outreach intervention to promote cervical cancer screening among Cambodian American women. Cancer Detect Prev. 2002a;26:320-7

57. *Taylor VM, Hislop TG, Jackson JC, Tu S-P, Yasui Y, Schwartz SM, et al. A Randomized Controlled Trial of Interventions to Promote Cervical Cancer Screening Among Chinese Women in North America. J Natl Cancer Inst. 2002b;94:670-7

58. *Taylor VM, Jackson JC, Yasui Y, Nguyen TT, Woodall E, Acorda E, et al. Evaluation of a Cervical Cancer Control Intervention Using Lay Health Workers for Vietnamese American Women. Am J Public Health. 2010;100:1924-9

59. *Walsh JME, Salazar R, Nguyen TT, Kaplan C, Nguyen L, Hwang J, et al. Healthy Colon, Healthy Life: A Novel Colorectal Cancer Screening Intervention. Am J Prev Med. 2010;39:1-14 60. *Zhu K, Hunter S, Bernard LJ, Payne-Wilks K, Roland CL, Elam LC, et al. An Intervention Study on Screening for Breast Cancer among Single African-American Women Aged 65 and Older. Prev Med. 2002;34:536-45

61. *Carney PA, Lee-Lin F, Mongoue-Tchokote S, Mori M, Leung H, Lau C, et al. Improving colorectal cancer screening in Asian Americans: Results of a randomized intervention study. Cancer. 2014; $120: 1702-12$

62. *Nguyen TT, Tsoh JY, Woo K, Stewart SL, Le GM, Burke A, et al. Colorectal Cancer Screening and Chinese Americans: Efficacy of Lay Health Worker Outreach and Print Materials. Am J Prev Med. 2017;52:e67-e76 63. *Tong EK, Nguyen TT, Lo P, Stewart SL, Gildengorin GL, Tsoh JY, et al. Lay health educators increase colorectal cancer screening among Hmong Americans: A cluster randomized controlled trial. Cancer. 2017;123:98-106

64. *Jo AM, Nguyen TT, Stewart S, Sung MJ, Gildengorin G, Tsoh JY, et al. Lay health educators and print materials for the promotion of colorectal cancer screening among Korean Americans: A randomized comparative effectiveness study. Cancer. 2017. Epub 2017 Apr 25 65. *Fernández ME, Gonzales A, Tortolero-Luna G, Williams J, Saavedra-Embesi M, Chan W, et al. Effectiveness of Cultivando La Salud: A Breast and Cervical Cancer Screening Promotion

44 Program for Low-Income Hispanic Women. Am J Public Health. 2009;99:936-43 
66. *Percac-Lima S, Grant RW, Green AR, Ashburner JM, Gamba G, Oo S, et al. A Culturally Tailored Navigator Program for Colorectal Cancer Screening in a Community Health Center: A Randomized, Controlled Trial. J Gen Intern Med. 2009;24:211-7 67. *Thompson B, Carosso EA, Jhingan E, Wang L, Holte SE, Byrd TL, et al. Results of a randomized controlled trial to increase cervical cancer screening among rural Latinas. Cancer. 2017; $123: 666-74$

68. *Duan N, Fox SA, Derose KP, Carson S. Maintaining mammography adherence through telephone counseling in a church-based trial. Am J Public Health. 2000;90:1468-71

69. Duggan C, Coronado G, Martinez J, Byrd TL, Carosso E, Lopez C, et al. Cervical cancer screening and adherence to follow-up among Hispanic women study protocol: a randomized controlled trial to increase the uptake of cervical cancer screening in Hispanic women. $B M C$ Cancer. 2012;12:170

70. *Castañeda SF, Xiong Y, Gallo LC, Yepes-Rios M, Ji M, Talavera AC, et al. Colorectal Cancer Educational Intervention Targeting Latino Patients Attending a Community Health Center. J Prim Care Community Health. 2012;3:164-9

71. *Sadler GR, Beerman PR, Lee K, Hung J, Nguyen H, Cho J, et al. Promoting Breast Cancer Screening Among Asian American Women: the Asian Grocery Store-Based Cancer Education Program. J Cancer Educ. 2012;27:612-7

72. *Potter MB, Namvargolian Y, Hwang J, Walsh JME. Improving Colorectal Cancer Screening: A Partnership Between Primary Care Practices and the American Cancer Society. $J$ Cancer Educ. 2009;24:22-7

73. Bandura. Social cognitive theory. Annals of Child Development. 1989;6:1-66

74. Swider SM, Martin M, Lynas C, Rothschild S. Project MATCH. Diabetes Educ. 2010;36:98-108

75. O'Brien MJ, Squires AP, Bixby RA, Larson SC. Role Development of Community Health Workers: An Examination of Selection and Training Processes in the Intervention Literature. Am J Prev Med. 2009;37:S262-S9

76. Michie S, Johnston M, Francis J, Hardeman W, Eccles M. From Theory to Intervention: Mapping Theoretically Derived Behavioural Determinants to Behaviour Change Techniques. Appl Psychol. 2008;57:660-80

77. Michie S, Johnston M. Theories and techniques of behaviour change: Developing a cumulative science of behaviour change. Health Psychol Rev. 2012;6:1-6

78. Foy R, Eccles M, Jamtvedt G, Young J, Grimshaw J, Baker R. What do we know about how to do audit and feedback? Pitfalls in applying evidence from a systematic review. BMC Health Serv Res. 2005;5:50

79. Forbes LJL, Simon AE, Warburton F, Boniface D, Brain KE, Dessaix A, et al. Differences in cancer awareness and beliefs between Australia, Canada, Denmark, Norway, Sweden and the UK (the International Cancer Benchmarking Partnership): do they contribute to differences in cancer survival[quest]. Br J Cancer. 2013;108:292-300

80. Whitaker KL, Macleod U, Winstanley K, Scott SE, Wardle J. Help seeking for cancer 'alarm' symptoms: a qualitative interview study of primary care patients in the UK. $\mathrm{Br} J \mathrm{Gen}$ Pract. 2015;65:e96-e105

81. Moffat J, Bentley A, Ironmonger L, Boughey A, Radford G, Duffy S. The impact of national cancer awareness campaigns for bowel and lung cancer symptoms on sociodemographic 
1 inequalities in immediate key symptom awareness and GP attendances. Br J Cancer.

2 2015; 112:S14-S21

3 82. Hoffmann TC, Glasziou PP, Boutron I, Milne R, Perera R, Moher D, et al. Better

4 reporting of interventions: template for intervention description and replication (TIDieR)

$5 \quad$ checklist and guide. BMJ. 2014;348:g1687

6 


\section{Page 69 of 95}

\section{Psycho-Oncology}

Table 1. Prevalence of behaviour change techniques in community-based health worker lay health led interventions

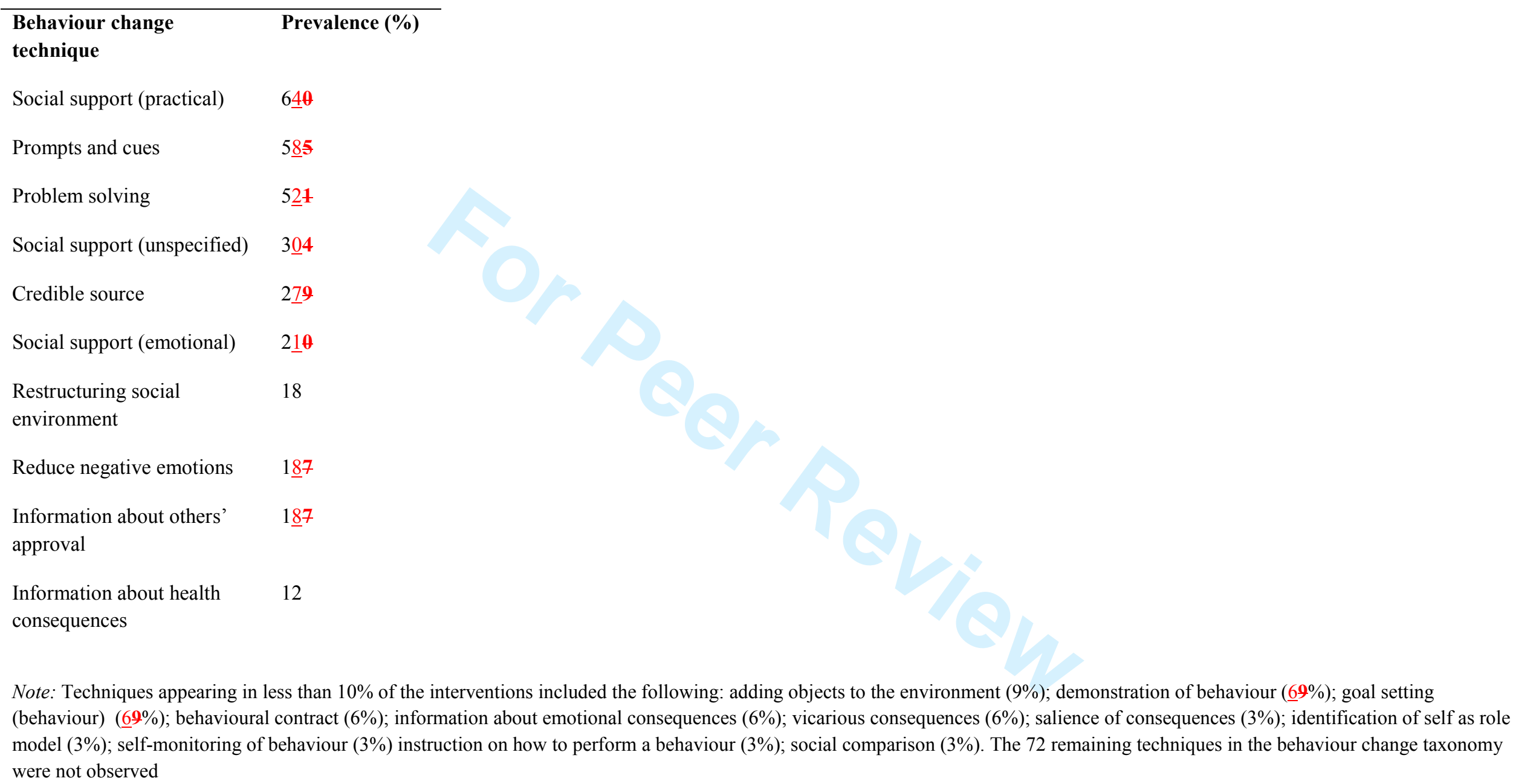


Table 2. Meta-analyses of studies reporting the effect of community-based lay health worker interventions on cancer screening

\begin{tabular}{|c|c|c|c|c|c|c|c|c|c|}
\hline & \multicolumn{3}{|l|}{ Sample } & \multicolumn{3}{|c|}{ Meta-analysis } & \multicolumn{3}{|c|}{ Heterogeneity } \\
\hline & Studies & Total $n$ & $\begin{array}{l}\text { Odds } \\
\text { Ratio }\end{array}$ & & $6 \mathrm{CI}$ & P value & Q-value & $\mathrm{P}$ value & $\mathrm{I}^{2}$ \\
\hline \multicolumn{10}{|l|}{ Primary and secondary outcomes } \\
\hline Primary: Overall screening adherence & 30 & 21990 & 1.901 & 1.60 & 2.26 & $<0.001$ & 199.9 & $<0.01$ & 85.5 \\
\hline $\begin{array}{l}\text { Secondary: Uptake among non- } \\
\text { adherent }\end{array}$ & 20 & 12768 & 2.401 & 1.85 & 3.11 & $<0.001$ & 149.1 & $<0.01$ & 87.3 \\
\hline \multicolumn{10}{|l|}{ Sensitivity analyses } \\
\hline Intention to treat data only & 19 & 16300 & 1.978 & 1.57 & 2.49 & $<0.001$ & 146.2 & $<0.01$ & 87.7 \\
\hline Medical records data only & 8 & 11658 & 2.092 & 1.36 & 3.21 & 0.001 & 112.6 & $<0.01$ & 93.8 \\
\hline Self-report data only & 18 & 8620 & 1.686 & 1.40 & 2.03 & $<0.001$ & 64.0 & $<0.01$ & 73.4 \\
\hline \multicolumn{10}{|l|}{ Subgroup analyses = type of procedure } \\
\hline Breast cancer (mammography) & 13 & 9641 & 1.923 & 1.44 & 2.57 & $<0.001$ & 96.7 & $<0.01$ & 87.6 \\
\hline Breast cancer (BSE) & 4 & 1493 & 1.554 & 1.17 & 2.07 & 0.003 & 4.96 & 0.175 & 39.5 \\
\hline Breast cancer (CBE) & 5 & 2582 & 1.528 & 1.19 & 2.00 & 0.001 & 8.76 & 0.067 & 54.4 \\
\hline Cervical cancer (pap test) & 15 & 5276 & 1.922 & 1.39 & 2.64 & $<0.001$ & 92.7 & $<0.01$ & 84.9 \\
\hline Colorectal (any) & 7 & 9425 & 1.983 & 1.31 & 3.01 & $<0.001$ & 86.8 & $<0.01$ & 93.1 \\
\hline \multicolumn{10}{|l|}{$\underline{\text { Subgroup analyses }=\text { delivery setting }}$} \\
\hline Community setting & 13 & 5621 & 2.249 & 1.63 & 3.11 & $<0.001$ & 83.7 & $<0.01$ & 85.7 \\
\hline Home-based setting & 13 & 5779 & 1.808 & 1.40 & 2.34 & $<0.001$ & 54.5 & $<0.01$ & 78.0 \\
\hline Primary care setting & $z$ & 6188 & 1.372 & 0.96 & 1.97 & 0.085 & 4.38 & 0.036 & 77.1 \\
\hline
\end{tabular}




\section{Page 71 of 95}

\section{Psycho-Oncology}

\begin{tabular}{|c|c|c|c|c|c|c|c|c|c|}
\hline Telephone өnly & $\underline{42}$ & $\frac{10590}{\mathbf{4 4 0 2}}$ & $1.3 \underline{22} 19$ & 1.14 & $1.5 \underline{4} 3$ & $<0.001$ & $\underline{50.914}$ & $\frac{0.116}{332}$ & $\frac{49.3}{\boldsymbol{\theta . 0 0}}$ \\
\hline \multicolumn{10}{|c|}{$\underline{\text { Subgroup analyses }=\text { intervention features }}$} \\
\hline Individual & 16 & 15256 & 1.680 & 1.40 & 2.02 & $<0.001$ & 63.1 & $<0.01$ & 76.2 \\
\hline Group-based & 5 & 2378 & 1.633 & 1.36 & 1.96 & $<0.001$ & 3.42 & 0.491 & 0.00 \\
\hline Individual + Group & 8 & 3636 & 2.463 & 1.43 & 4.23 & 0.001 & 93.8 & $<0.01$ & 92.5 \\
\hline BCTs; 3 or less & 17 & 15433 & 1.660 & 1.34 & 2.05 & $<\mathbf{0 . 0 0 1}$ & 105.5 & 0.001 & 84.8 \\
\hline BCTs; 4 or more & 13 & 6557 & 2.268 & 1.78 & 2.89 & $<0.001$ & 50.057 & 0.001 & 76.0 \\
\hline
\end{tabular}

Note: BSE, breast self-examination; CBE, clinical breast examination; ITT, intention to treat BOLD represents statistically significant benefit of $\mathrm{LCBHW}$ interventions. 


\section{Figure legends}

Figure 1. PRISMA flow diagram of study selection

Figure 2. Meta-analysis of cancer screening adherence following $\underline{\mathrm{LCBHW}}$ interventions in comparison to control conditions. Box size represents study weighting. Diamond represents overall effect size and 95\% confidence intervals. 
Appendix S1. Database search strategies

\section{MEDLINE}

((((volunteer*) [TI] OR (("expert patient*") [TIAB] OR (natural ADJ2 helper*) [TIAB] OR (volunteer* ADJ5 (worker* OR people OR person* OR helper* OR educator OR adviser* OR advisor*)) [TIAB] OR (untrained ADJ5 (worker* OR people OR person* OR helper* OR educator* OR adviser* OR advisor)) [TIAB] OR (voluntary* ADJ5 (worker* OR people OR person* OR helper* OR educator* OR adviser* OR advisor)) [TIAB] OR (lay ADJ5 (worker* OR people OR person* OR helper* OR educator* OR adviser* OR advisor)) [TIAB] OR (community ADJ5 (worker* OR people OR person* OR helper* OR educator* OR adviser* OR advisor)) [TIAB] OR (peer ADJ5 (worker* OR people OR person* OR helper* OR educator* OR adviser* OR advisor)) [TIAB] OR exp VOLUNTEERS/) AND (("early diagnos*") [TIAB] OR ("early detection") [TIAB] OR (symptom*) [TIAB] OR ("risk factor*") [TIAB] OR exp "RISK FACTORS"/ OR ("screening program*") [TIAB] OR exp "EARLY DETECTION OF CANCER"/ OR ("help seeking behaviour*") [TIAB] OR ("help seeking behavior*") [TIAB] OR (confidence) [TIAB] OR (self-efficacy) [TIAB] OR exp "SELF EFFICACY"/)) AND ((tumour*) [TIAB] OR (cancer*) [TIAB] OR (neoplas*) [TIAB] OR (oncolog*) [TIAB] OR (melanom*) [TIAB] OR (maligna*) [TIAB] OR (gliom*) [TIAB] OR (carcin*) [TIAB] OR (metasta*)[TIAB] OR (sarcom*)[TIAB] OR (tumor*) [TIAB] OR chemotherap* [TIAB] OR (leukaem [TIAB] OR exp NEOPLASMS/ OR (leukem*) [TIAB] OR (radiotherap*) [TIAB] OR (lymphom*) [TIAB] OR (mesotheliom*)[TIAB] NOT ("clinical trial*") [TIAB]

\section{Embase}

$(((($ volunteer*) $[\mathrm{TI}]$ OR (volunteer* ADJ5 (worker* OR people OR person* OR helper* OR educator* OR adviser* OR advisor)) [TIAB] OR (untrained ADJ5 (worker* OR people OR person* OR helper* OR educator* OR adviser* OR advisor)) [TIAB] OR (voluntary* ADJ5 (worker* OR people OR person* OR helper* OR educator* OR adviser* OR advisor)) [TIAB] OR (lay ADJ5 (worker* OR people OR person* OR helper* OR educator* OR adviser* OR advisor)) [TIAB] OR (community ADJ5 (worker* OR people OR person* OR helper* OR educator* OR adviser* OR advisor)) [TIAB] OR (peer ADJ5 (worker* OR people OR person* OR helper* OR educator* OR adviser* OR advisor)) [TIAB] OR exp VOLUNTEERS/ OR ("expert patient*") [TIAB] OR (natural ADJ2 helper) [TIAB] AND ((tumour*) [TIAB] OR (cancer*) [TIAB] OR (neoplas*) [TIAB] OR (oncolog*) [TIAB] OR (melanom*) [TIAB] OR (maligna*) [TIAB] OR (gliom*) [TIAB] OR (carcin*) [TIAB] OR (metasta*) [TIAB] OR (sarcom*) [TIAB] OR (tumor*) [TIAB] OR chemotherap* [TIAB] OR (leukaem*) [TIAB] OR (leukem*) [TIAB] OR (radiotherap* [TIAB] OR (lymphom*) [TIAB] OR (mesotheliom*) [TIAB] OR exp NEOPLASM/)) AND (("early diagnos*") [TIAB] OR ("early detection") [TIAB] OR (symptom*) [TIAB] OR ("risk factor*") [TIAB] OR exp "RISK FACTORS"/ OR ("screening program*") [TIAB] OR exp "CANCER SCREENING"/ OR ("help seeking behaviour*") [TIAB] OR ("help seeking behavior*") [TIAB] OR (confidence) [TIAB] OR (selfefficacy) [TIAB] OR exp "SELF ESTEEM"/)) NOT (("clinical trial*") [TIAB] OR exp "CLINICAL TRIAL (TOPIC)"/))

\section{BNI}

((((volunteer*) [TI] OR (volunteer* ADJ5 (worker* OR people OR person* OR helper* OR educator* OR adviser* OR advisor)) [TIAB] OR (untrained ADJ5 (worker* OR people OR person* 
OR helper* OR educator* OR adviser* OR advisor)) [TIAB] OR (voluntary* ADJ5 (worker* OR people OR person* OR helper* OR educator* OR adviser* OR advisor)) [TIAB] OR (lay ADJ5 (worker* OR people OR person* OR helper* OR educator* OR adviser* OR advisor)) [TIAB] OR exp "VOLUNTARY ORGANISATIONS"/ OR ("expert patient*") [TIAB] OR (community ADJ5 (worker* OR people OR person* OR helper* OR educator* OR adviser* OR advisor)) [TIAB] OR (natural ADJ2 helper*) [TIAB] AND ((tumour*) [TIAB] OR (cancer*) [TIAB] OR (neoplas*) [TIAB] OR (oncolog*) [TIAB] OR (melanom*) [TIAB] OR (maligna*) [TIAB] OR (gliom*) [TIAB] OR (carcin*) [TIAB] OR (metasta*) [TIAB] OR (sarcom*) [TIAB] OR (tumor*) [TIAB] OR chemotherap* OR (leukaem*)[TIAB] OR (leukem*) [TIAB] OR (radiotherap*) [TIAB] OR (lymphom*) [TIAB] OR (mesotheliom*) [TIAB] OR exp CANCER/)) AND (("early diagnos*") [TIAB] OR ("early detection") [TIAB] OR ("symptom*") [TIAB] OR ("risk factor*") [TIAB] OR exp EPIDEMIOLOGY/ OR ("screening program*") [TIAB] OR exp "CANCER : PREVENTION AND SCREENING"/ OR ("help seeking behaviour*") [TIAB] OR ("help seeking behavior*") [TIAB] OR (confidence) [TIAB] OR (self-efficacy) [TIAB]))

\section{CINAHL}

(((volunteer*) [TI] OR (volunteer* ADJ5 (worker* OR people OR person* OR helper* OR educator* OR adviser* OR advisor)) [TIAB] OR (untrained ADJ5 (worker* OR people OR person* OR helper* OR educator* OR adviser* OR advisor)) [TIAB] OR (voluntary* ADJ5 (worker* OR people OR person* OR helper* OR educator* OR adviser* OR advisor)) [TIAB] OR (lay ADJ5 (worker* OR people OR person* OR helper* OR educator* OR adviser* OR advisor)) [TIAB] OR (community ADJ5 (worker* OR people OR person* OR helper* OR educator* OR adviser* OR advisor)) [TIAB] OR (peer ADJ5 (worker* OR people OR person* OR helper* OR educator* OR adviser* OR advisor)) [TIAB] OR exp "VOLUNTEER WORKERS"/ OR exp "VOLUNTEER EXPERIENCES"/ OR ("expert patient*") [TIAB] OR (natural ADJ2 helper*) [TIAB]) AND (exp NEOPLASMS/ OR ((tumour*) [TIAB] OR (cancer*) [TIAB] OR (neoplas*) [TIAB] OR (oncolog*) [TIAB] OR (melanom*) [TIAB] OR (maligna*) [TIAB] OR (gliom*) [TIAB] OR (carcin*) [TIAB] OR (metasta*) [TIAB] OR (sarcom*) [TIAB] OR (tumor*) [TIAB] OR chemotherap* [TIAB] OR (leukaem*) [TIAB]) OR (leukem*) [TIAB] OR (radiotherap*) [TIAB] OR (lymphom*) [TIAB] OR (mesotheliom*) [TIAB])) AND (("early diagnos*") [TIAB] OR ("early detection") [TIAB] OR (symptom*) [TIAB] OR ("risk factor*") [TIAB] OR exp "RISK FACTORS"/ OR ("screening program*") [TIAB] OR exp "CANCER SCREENING"/ OR ("help seeking behaviour*") [TIAB] OR ("help seeking behavior*") [TIAB] OR (confidence) [TIAB] OR exp "SELFCONFIDENCE"/ OR (self-efficacy) [TIAB]))

\section{PsycINFO}

$(((($ volunteer*) [TI] OR (volunteer* ADJ5 (worker* OR people OR person* OR helper* OR educator* OR adviser* OR advisor)) [TIAB] OR (untrained* ADJ5 (worker* OR people OR person* OR helper* OR educator* OR adviser* OR advisor)) [TIAB] OR (voluntary* ADJ5 (worker* OR people OR person* OR helper* OR educator* OR adviser* OR advisor)) [TIAB] OR (lay ADJ5 (worker* OR people OR person* OR helper* OR educator* OR adviser* OR advisor)) [TIAB] OR (peer ADJ5 (worker* OR people OR person* OR helper* OR educator* OR adviser* OR advisor)) [TIAB] OR (community ADJ5 (worker* OR people OR person* OR helper* OR educator* OR adviser* OR advisor)) [TIAB] OR exp VOLUNTEERS/ OR ("expert patient*") [TIAB] OR (natural ADJ2 helper*) [TIAB]) AND ((tumour*) [TIAB] OR (cancer*) [TIAB] OR (neoplas*) [TIAB] OR (oncolog*) [TIAB] OR (melanom*) [TIAB] OR (maligna*) [TIAB] OR(gliom*) [TIAB] OR 
(carcin*) [TIAB] OR (metasta*) [TIAB] OR (sarcom*) [TIAB] OR (tumor*) [TIAB] OR chemotherap* [TIAB] OR (leukaem*) [TIAB] OR (leukem*) [TIAB] OR (radiotherap*) [TIAB] OR (lymphom*) [TIAB] OR (mesotheliom*) [TIAB] OR exp NEOPLASMS/)) AND (("early diagnos*") [TIAB] OR ("early detection") [TIAB] OR (symptom*) [TIAB] OR ("risk factor*") [TIAB] OR exp "RISK FACTORS"/ OR ("screening program*") [TIAB] OR exp "CANCER SCREENING"/ OR ("help seeking behaviour*") [TIAB] OR ("help seeking behavior*") [TIAB] OR (confidence) [TIAB] OR exp "SELFCONFIDENCE"/ OR exp "SELF-EFFICACY"/ OR (self-efficacy) [TIAB]))

\begin{abstract}
AMED
((((volunteer*) [TI] OR (volunteer* ADJ5 (worker* OR people OR person* OR helper* OR educator* OR adviser* OR advisor)) [TIAB] OR (untrained* ADJ5 (worker* OR people OR person* OR helper* OR educator* OR adviser* OR advisor)) [TIAB] OR (voluntary* ADJ5 (worker* OR people OR person* OR helper* OR educator* OR adviser* OR advisor)) [TIAB] OR (lay ADJ5 (worker* OR people OR person* OR helper* OR educator* OR adviser* OR advisor)) [TIAB] OR (peer ADJ5 (worker* OR people OR person* OR helper* OR educator* OR adviser* OR advisor)) [TIAB] OR (community ADJ5 (worker* OR people OR person* OR helper* OR educator* OR adviser* OR advisor)) [TIAB] OR exp VOLUNTEERS/ OR ("expert patient*") [TIAB] OR (natural ADJ2 helper*) [TIAB]) AND AND ((tumour*) [TIAB] OR (cancer*) [TIAB] OR (neoplas*) [TIAB] OR (oncolog*) [TIAB] OR (melanom*) [TIAB] OR (maligna*) [TIAB] OR(gliom*) [TIAB] OR (carcin*) [TIAB] OR (metasta*) [TIAB] OR (sarcom*) [TIAB] OR (tumor*) [TIAB] OR chemotherap* [TIAB] OR (leukaem*) [TIAB] OR (leukem*) [TIAB] OR (radiotherap*) [TIAB] OR (lymphom*) [TIAB] OR (mesotheliom*) [TIAB] OR exp NEOPLASMS/)) AND (("early diagnos*") [TIAB] OR ("early detection") [TIAB] OR (symptom*) [TIAB] OR ("risk factor*") [TIAB] OR exp "RISK FACTORS"/ OR ("screening program*") [TIAB] OR exp "CANCER SCREENING"/ OR ("help seeking behaviour*") [TIAB] OR ("help seeking behavior*") [TIAB] OR (confidence) [TIAB] OR exp "SELFCONFIDENCE"/ OR (self-efficacy) [TIAB]))
\end{abstract}


Appendix 2. Template data extraction form

\begin{tabular}{|c|c|c|c|c|}
\hline \multicolumn{5}{|l|}{ Date of data extraction: } \\
\hline \multicolumn{5}{|l|}{ Name of reviewer: } \\
\hline \multicolumn{5}{|l|}{ Paper title: } \\
\hline \multicolumn{3}{|l|}{ First Author: } & \multicolumn{2}{|l|}{ Year/volume/pages: } \\
\hline \multicolumn{5}{|l|}{ Comments: } \\
\hline Participants & Control group & Group 1 & Group 2 & Overall \\
\hline \multicolumn{5}{|l|}{$\begin{array}{l}\text { No. of included } \\
\text { participants (those } \\
\text { randomized) }\end{array}$} \\
\hline \multicolumn{5}{|l|}{ Mean age (SD) } \\
\hline \multicolumn{5}{|l|}{ No. of males } \\
\hline \multicolumn{5}{|l|}{ No. of females } \\
\hline \multicolumn{5}{|l|}{$\begin{array}{l}\text { Ethnicity: } \\
\text { White (No.) } \\
\text { Non-white (No.) }\end{array}$} \\
\hline $\begin{array}{l}\text { No of participants at each } \\
\text { follow up stage }\end{array}$ & & & & \\
\hline
\end{tabular}

\section{Sample size}

\section{Is a power calculation performed?}

Yes $\square$

No $\square$

If "no" proceed to intervention characteristics.

Is the power estimate achieved?

Yes $\square$

No $\square$
Justification given for sample size:

Further detail:

\begin{tabular}{|l|l|}
\hline Intervention characteristics \\
\hline $\begin{array}{l}\text { Design of study: } \\
\text { Randomised controlled trial } \square\end{array}$ & Further detail: \\
\hline
\end{tabular}




\begin{tabular}{|c|c|}
\hline Cluster randomised controlled trial $\square$ & \\
\hline $\begin{array}{l}\text { Control group type: } \\
\text { Active control } \square \\
\text { Delayed intervention } \square \\
\text { Alternative intervention } \square \\
\text { Usual care } \square \\
\text { No intervention } \square\end{array}$ & Details of what control group received: \\
\hline $\begin{array}{l}\text { Setting (Tick all that apply): } \\
\text { Workplace } \square \\
\text { Community setting } \square \\
\text { Participant's home } \square \\
\text { Primary care practice } \square \\
\text { Outpatient clinic } \square \\
\text { Telephone } \square \\
\text { Unclear } \square \\
\text { Other (specify): }\end{array}$ & Further information: \\
\hline $\begin{array}{l}\text { Intervention type: } \\
\text { Individual } \square \\
\text { Group } \square \\
\text { Combination of individual and group } \square\end{array}$ & Further information e.g. how many if group: \\
\hline Number of intervention contact sessions: & \\
\hline Duration of sessions: & \\
\hline $\begin{array}{l}\text { Content of any additional components to the } t \mathrm{CB} H W \text { (Tick all that } \\
\text { apply): } \\
\text { Computer element } \square \\
\text { Media } \square \\
\text { No other components } \square\end{array}$ & $\sqrt{20}+x^{2}$ \\
\hline $\begin{array}{l}\text { Was there a follow up component to the intervention? } \\
\text { Yes } \square \\
\text { No } \square \\
\text { If "no", proceed to outcome measures. }\end{array}$ & Further detail: \\
\hline $\begin{array}{l}\text { Mode of follow up component (Tick all that apply): } \\
\text { Media (e.g. educational pamphlets) } \square \\
\text { Telephone call } \square \\
\text { In person visit } \square \\
\text { Unclear } \square\end{array}$ & \\
\hline $\begin{array}{l}\text { Content of follow up component (Tick all that apply): } \\
\text { Logistic/navigational assistance (e.g. making appointments, arranging } \\
\text { transportation, accompanying someone to screening) } \\
\text { Screening reminder } \square \\
\text { Educational brochure } \square \\
\text { Counselling on barriers } \square\end{array}$ & Further detail: \\
\hline
\end{tabular}




\begin{tabular}{|l|l|}
\hline Outcome measures \\
\hline $\begin{array}{l}\text { Primary outcome measures: } \\
\text { Screening uptake } \square \\
\text { Intention to screen } \square\end{array}$ & $\begin{array}{l}\text { Measurement tool: } \\
\text { Self-report } \square \\
\text { Medical records } \\
\text { Combination of both } \square\end{array}$ \\
\hline $\begin{array}{l}\text { Secondary outcome measures: } \\
\text { Knowledge/awareness } \square \\
\text { Beliefs/attitude } \square \\
\text { Help-seeking behaviour } \square \\
\text { None } \square \\
\text { Other (specify): }\end{array}$ & \\
\hline
\end{tabular}

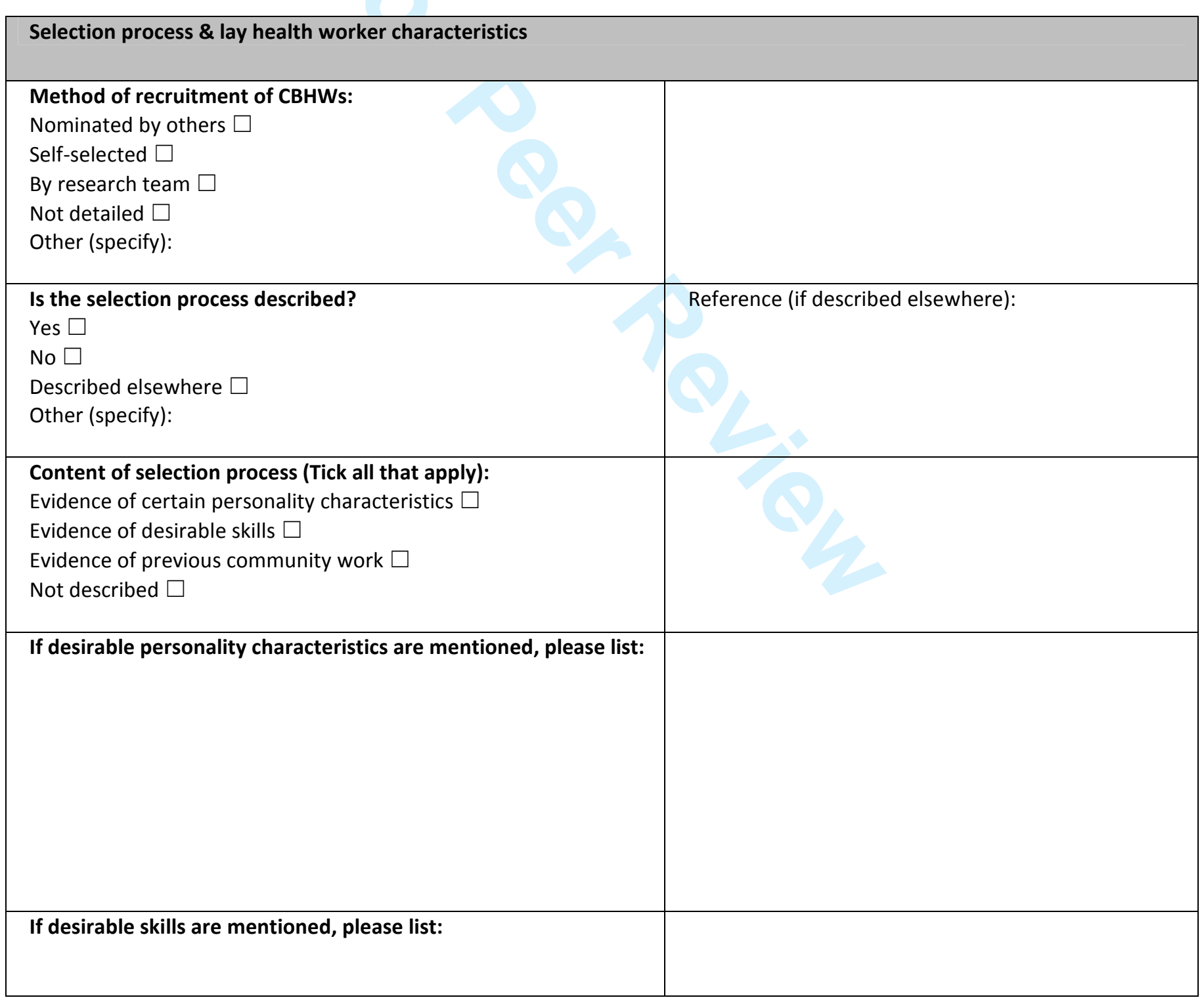


Further details about $t \underline{C B H W s}$ (e.g. if they are a survivor, ethnicity, SES):

\section{Gender of $\underline{t} \underline{C B} H W s$ :}

Female $\square$

Male $\square$

Male \& female

Unknown $\square$

\begin{tabular}{|c|c|}
\hline \multicolumn{2}{|l|}{ Training } \\
\hline $\begin{array}{l}\text { Is training for the } \underline{\underline{L} \mathrm{CB} H W \text { m mentioned? }} \\
\text { Yes } \square \\
\text { No } \square \\
\text { If "no" proceed to quality/competence assessment. }\end{array}$ & \\
\hline $\begin{array}{l}\text { Training is delivered by (Tick all that apply): } \\
\text { Members of the research team } \square \\
\text { Healthcare professional } \square \\
\text { Students } \square \\
\text { Unknown } \square \\
\text { Other (specify): }\end{array}$ & Further detail: \\
\hline Duration of training: & \\
\hline $\begin{array}{l}\text { Evidence of booster training sessions throughout intervention period: } \\
\text { Yes } \square \\
\text { No } \square\end{array}$ & Further detail: \\
\hline $\begin{array}{l}\text { Evidence of quality/competence assessment (Tick all that apply): } \\
\text { Direct observation immediately after training (e.g. practice session) } \\
\text { Sporadic observation during intervention period } \square \\
\text { Quality checking forms } \square \\
\text { On-going supervision } \square \\
\text { Written examination } \square \\
\text { Pre-test and post-test of knowledge } \square \\
\text { No evidence of a quality/competence assessment } \square \\
\text { Other (specify): }\end{array}$ & Further detail: \\
\hline $\begin{array}{l}\text { Content of training (Tick all that apply): } \\
\text { Curriculum manual } \square \\
\text { Research skills (e.g. randomisation, recruitment, protocol adherence) } \square \\
\text { Communication skills } \square \\
\text { Lectures } \square \\
\text { Didactic role-plays } \square\end{array}$ & Further detail: \\
\hline
\end{tabular}


Group discussions $\square$

Health knowledge $\square$

Taught how to tailor information $\square$

Practice intervention sessions $\square$

Modelling techniques $\square$

Motivational skills $\square$

Visual aids/flip charts $\square$

How to provide logistical/navigational assistance

Not detailed $\square$

Other (specify):

\begin{tabular}{|c|c|c|}
\hline Risk of bias (Cochrane Handbook) & $\begin{array}{l}\text { Judgement of risk } \\
\text { (High/Low/Unclear) }\end{array}$ & Support for judgement \\
\hline \multicolumn{3}{|l|}{ Selection bias } \\
\hline \multicolumn{3}{|l|}{ Random sequence generation } \\
\hline \multicolumn{3}{|l|}{ Allocation concealment } \\
\hline \multicolumn{3}{|l|}{ Performance bias } \\
\hline \multicolumn{3}{|l|}{ Blinding of participants and personnel } \\
\hline \multicolumn{3}{|l|}{ Detection bias } \\
\hline \multicolumn{3}{|l|}{ Blinding of outcome assessment } \\
\hline \multicolumn{3}{|l|}{ Attrition bias } \\
\hline \multicolumn{3}{|l|}{ Incomplete outcome data } \\
\hline \multicolumn{3}{|l|}{ Reporting bias } \\
\hline \multicolumn{3}{|l|}{ Selective reporting } \\
\hline \multicolumn{3}{|l|}{ Other bias } \\
\hline Other sources of bias & & \\
\hline
\end{tabular}

\section{Analysis}

Time point of main analysis: 


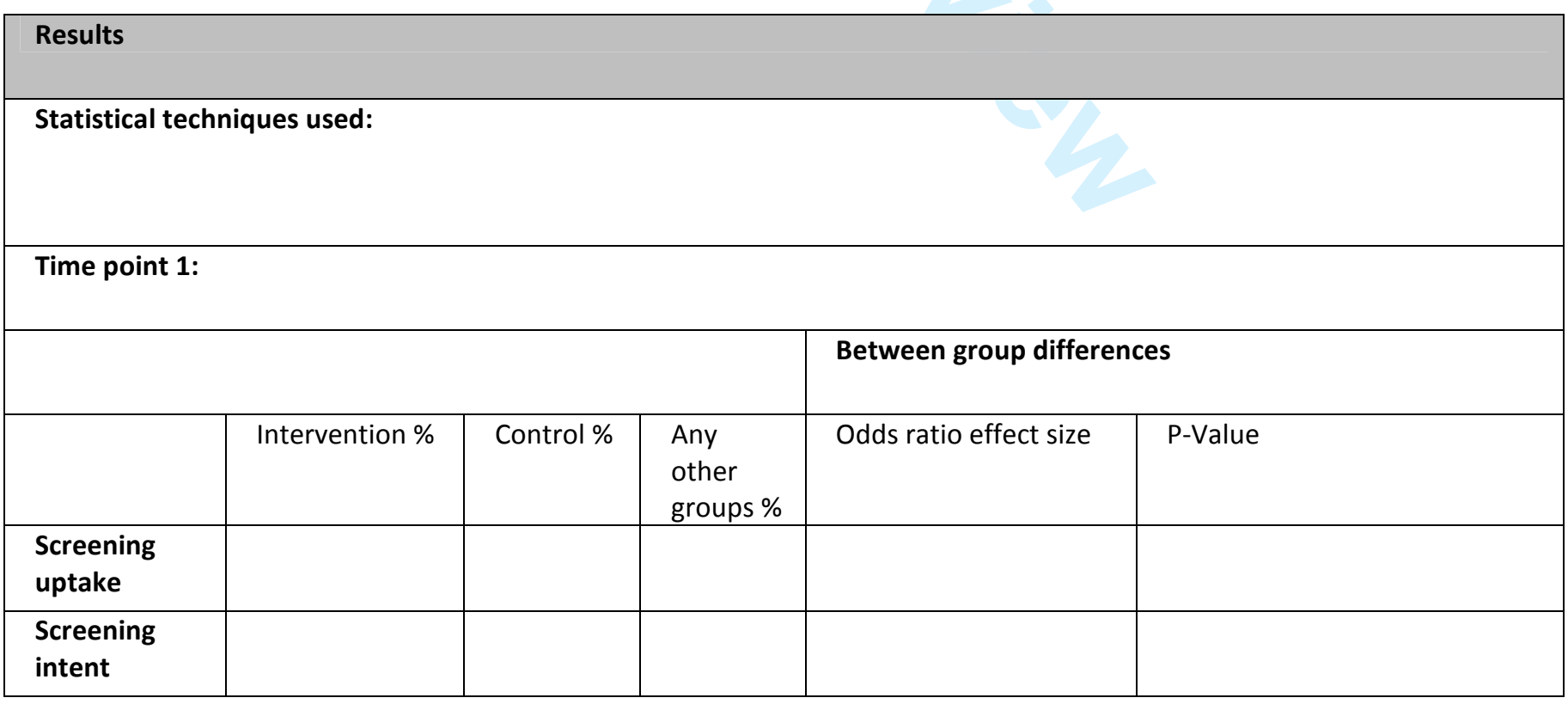

\section{Reasons for drop out:}

Excluded by researcher

Dropped out from intervention $\square$

Could not be contacted $\square$

Non-completion of measures

Outliers $\square$

Ineligible after baseline interview $\square$

Refused to participate

No attrition $\square$

Not detailed $\square$

Other (specify):

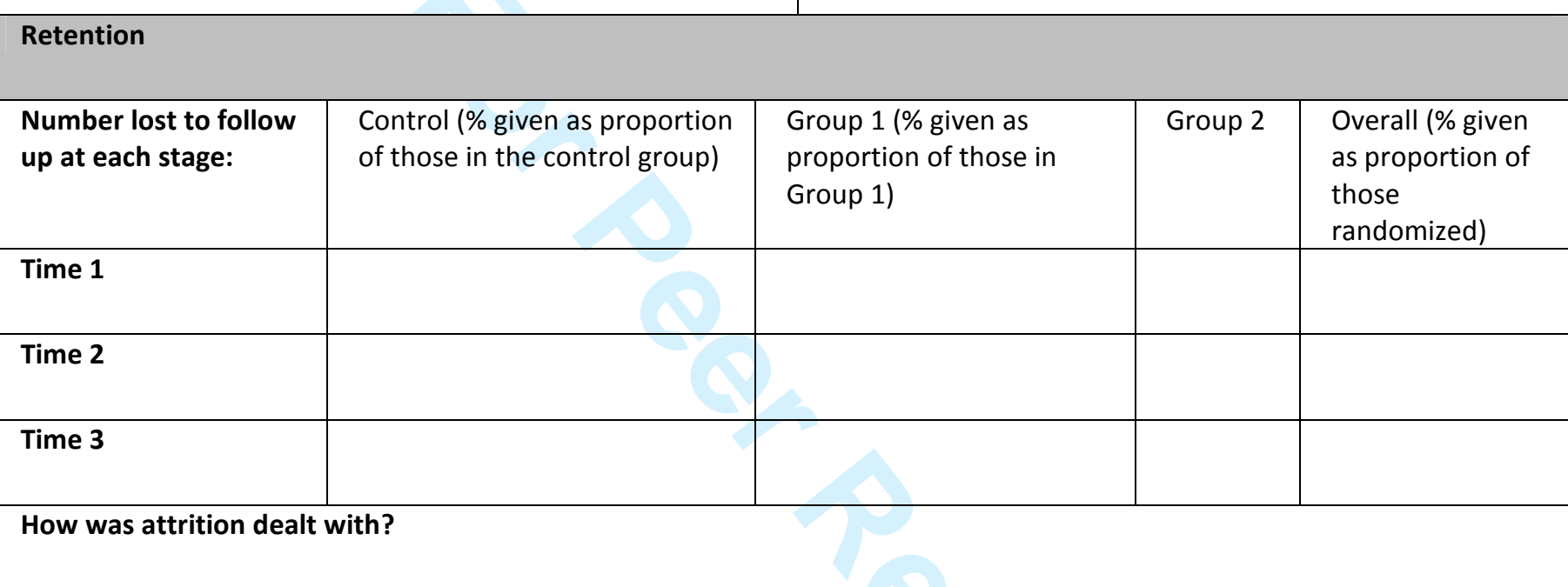

Further details: 


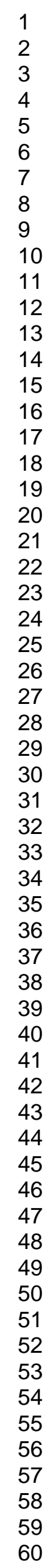

http://mc.manuscriptcentral.com/pon 
Appendix 3. Study details and intervention summaries

\begin{tabular}{|c|c|c|c|c|c|c|c|c|c|c|}
\hline \multirow[b]{2}{*}{$\begin{array}{c}\text { First } \\
\text { author } \\
\text { (ref.) }\end{array}$} & \multicolumn{3}{|c|}{ Study population and setting } & \multicolumn{3}{|c|}{ Outcome measure } & \multicolumn{4}{|c|}{ Intervention description } \\
\hline & $\begin{array}{c}\text { Target } \\
\text { population }\end{array}$ & Age range $\dagger$ & $\begin{array}{l}\text { Study } \\
\text { setting }\end{array}$ & $\begin{array}{c}\text { Type of } \\
\text { cancer } \\
\text { screening }\end{array}$ & $\begin{array}{c}\text { Follow up } \\
\text { duration }\end{array}$ & $\begin{array}{l}\text { Data source for } \\
\text { screening } \\
\text { outcome }\end{array}$ & $\begin{array}{l}\text { Delivery } \\
\text { mode }\end{array}$ & $\begin{array}{l}\text { Intervention } \\
\text { components }\end{array}$ & $\begin{array}{l}\text { No. of } \\
\text { BCTs }\end{array}$ & $\begin{array}{l}\text { Theoretic } \\
\text { framework }\end{array}$ \\
\hline & \multicolumn{10}{|c|}{ Cancer screening } \\
\hline $\begin{array}{l}\text { Byrd } \\
(2013)\end{array}$ & Hispanic women & Not reported & Community & Cervical & $6 \mathrm{mths}$ & $\mathrm{MR}+\mathrm{SR}$ & $\begin{array}{l}\text { Individual } \\
\text { (face to face) }\end{array}$ & $\begin{array}{l}\text { Flipchart, educational } \\
\text { information, video }\end{array}$ & 8 & $\begin{array}{l}\text { SCT, HBM, TTM, } \\
\text { TRA }\end{array}$ \\
\hline $\begin{array}{l}\text { Carney } \\
(2014)\end{array}$ & $\begin{array}{l}\text { Asian American } \\
\text { men and women }\end{array}$ & $46-91$ & Community & Colorectal & $\begin{array}{l}\text { Immediately } \\
\text { post } \\
\text { intervention }\end{array}$ & SR (intention) & $\begin{array}{l}\text { Group (face to } \\
\text { face) }\end{array}$ & $\begin{array}{l}\text { Educational information, } \\
\text { logistical assistance, } \\
\text { barriers counselling }\end{array}$ & 2 & N/A \\
\hline $\begin{array}{l}\text { Castañeda } \\
(2012)\end{array}$ & $\begin{array}{l}\text { Hispanic men } \\
\text { and women }\end{array}$ & $64(8.0)$ & $\begin{array}{l}\text { Primary } \\
\text { care }\end{array}$ & Colorectal & $\begin{array}{l}\text { Immediately } \\
\text { post } \\
\text { intervention }\end{array}$ & SR & $\begin{array}{l}\text { Group (face to } \\
\text { face) }\end{array}$ & $\begin{array}{l}\text { Educational information, } \\
\text { educational pamphlet }\end{array}$ & 1 & N/A \\
\hline $\begin{array}{l}\text { Dulan } \\
(2000)\end{array}$ & $\begin{array}{l}\text { White, African } \\
\text { American and } \\
\text { Hispanic women }\end{array}$ & $50-80$ & $\begin{array}{l}\text { Telephone } \\
\text { Home } \\
\text { (telephone) }\end{array}$ & Breast & $12 \mathrm{mths}$ & SR & $\begin{array}{l}\text { Individual } \\
\text { (telephone) }\end{array}$ & $\begin{array}{l}\text { Educational information, } \\
\text { barriers counselling, } \\
\text { logistical assistance }\end{array}$ & 1 & HBM \\
\hline $\begin{array}{l}\text { Fernandez } \\
(2009)\end{array}$ & Hispanic women & $50-70+$ & Home & $\begin{array}{l}\text { Breast, } \\
\text { Cervical }\end{array}$ & $6 \mathrm{mths}$ & $\mathrm{MR}+\mathrm{SR}$ & $\begin{array}{l}\text { Individual } \\
\text { (face to face } \\
\text { \& telephone) }\end{array}$ & $\begin{array}{l}\text { Educational information, } \\
\text { DVD, survivor narratives, } \\
\text { logistical assistance, } \\
\text { follow up reminder }\end{array}$ & 1 & N/A \\
\hline $\begin{array}{l}\text { Golelen } \\
(2010)\end{array}$ & Flemish women & $50-69$ & $\begin{array}{l}\text { Telephone } \\
\text { Home } \\
\text { (telephone) }\end{array}$ & Breast & 4-6 weeks & MR & $\begin{array}{l}\text { Individual } \\
\text { (telephone) }\end{array}$ & $\begin{array}{l}\text { Educational pamphlet, } \\
\text { invitation letter for } \\
\text { screening mammography, } \\
\text { telephone reminder }\end{array}$ & 2 & N/A \\
\hline
\end{tabular}


Appendix 3. Study details and intervention summaries (Cont'd)

\begin{tabular}{|c|c|c|c|c|c|c|c|c|c|c|}
\hline \multirow[b]{2}{*}{$\begin{array}{c}\text { First } \\
\text { author } \\
\text { (ref.) }\end{array}$} & \multicolumn{3}{|c|}{ Study population and setting } & \multicolumn{3}{|c|}{ Outcome measure } & \multicolumn{4}{|c|}{ Intervention description } \\
\hline & $\begin{array}{c}\text { Target } \\
\text { population }\end{array}$ & Age range $\dagger$ & $\begin{array}{l}\text { Study } \\
\text { setting }\end{array}$ & $\begin{array}{l}\text { Type of } \\
\text { cancer } \\
\text { screening }\end{array}$ & $\begin{array}{c}\text { Follow up } \\
\text { duration }\end{array}$ & $\begin{array}{l}\text { Data source for } \\
\text { screening } \\
\text { outcome }\end{array}$ & $\begin{array}{l}\text { Delivery } \\
\text { mode }\end{array}$ & Intervention components & $\begin{array}{l}\text { No. of } \\
\text { BCTs }\end{array}$ & $\begin{array}{l}\text { Theoretic } \\
\text { framework }\end{array}$ \\
\hline Han (2017) & $\begin{array}{l}\text { Asian American } \\
\text { women }\end{array}$ & $21-64$ & Community & $\begin{array}{l}\text { Breast, } \\
\text { Cervical }\end{array}$ & $6 \mathrm{mths}$ & MR & $\begin{array}{l}\text { Group (face to } \\
\text { face) }+ \\
\text { individual } \\
\text { (telephone) }\end{array}$ & $\begin{array}{l}\text { Educational information, } \\
\text { educational pamphlet, } \\
\text { DVD, logistical assistance, } \\
\text { follow up reminder }\end{array}$ & 2 & N/A \\
\hline $\begin{array}{l}\text { Hunter } \\
(2004)\end{array}$ & Hispanic women & $40-70$ & Home & $\begin{array}{l}\text { Breast, } \\
\text { Cervical, } \\
\text { CBE }\end{array}$ & Unclear & MR & $\begin{array}{l}\text { Individual } \\
\text { (face to face) }\end{array}$ & $\begin{array}{l}\text { Educational pamphlets, } \\
\text { logistical assistance, follow } \\
\text { up reminder }\end{array}$ & 4 & N/A \\
\hline $\begin{array}{l}\text { Jandorf } \\
(2008)\end{array}$ & Hispanic women & $38.8(13.8)$ & Community & $\begin{array}{l}\text { Breast, } \\
\text { Cervical, } \\
\text { BSE, CBE }\end{array}$ & $2 \mathrm{mths}$ & SR & $\begin{array}{l}\text { Group (face to } \\
\text { face) }\end{array}$ & $\begin{array}{l}\text { Educational information, } \\
\text { survivor narratives, } \\
\text { logistical assistance }\end{array}$ & 3 & N/A \\
\hline Jo (2017) & $\begin{array}{l}\text { Asian American } \\
\text { men and women }\end{array}$ & $61.4(7.6)$ & Community & Colorectal & $6 \mathrm{mths}$ & SR & $\begin{array}{l}\text { Group (face to } \\
\text { face) }+ \\
\text { individual } \\
\text { (telephone) }\end{array}$ & $\begin{array}{l}\text { Flipchart, educational } \\
\text { information, educational } \\
\text { pamphlet, barriers } \\
\text { counselling, follow up } \\
\text { reminder }\end{array}$ & 5 & SCT, TTM \\
\hline Lam (2003) & $\begin{array}{l}\text { Asian American } \\
\text { women }\end{array}$ & $18-88$ & Community & Cervical & 3-4 mths & SR & $\begin{array}{l}\text { Group (face to } \\
\text { face) }+ \\
\text { individual } \\
\text { (telephone) }\end{array}$ & $\begin{array}{l}\text { Flipchart, educational } \\
\text { information, logistical } \\
\text { assistance, follow up } \\
\text { reminder, media campaign }\end{array}$ & 2 & $\mathrm{~N} / \mathrm{A}$ \\
\hline $\begin{array}{l}\text { Mock } \\
(2007)\end{array}$ & $\begin{array}{l}\text { Asian American } \\
\text { women }\end{array}$ & $18-88$ & Community & Cervical & $3-4$ mths & SR & $\begin{array}{l}\text { Group (face to } \\
\text { face) }+ \\
\text { individual } \\
\text { (telephone) }\end{array}$ & $\begin{array}{l}\text { Flipchart, educational } \\
\text { information, follow up } \\
\text { reminder, logistical } \\
\text { assistance, media campaign }\end{array}$ & 2 & N/A \\
\hline
\end{tabular}




\begin{tabular}{|c|c|c|c|c|c|c|c|c|c|c|}
\hline \multicolumn{11}{|c|}{ Appendix 3. Study details and intervention summaries (Cont'd) } \\
\hline \multirow[b]{2}{*}{$\begin{array}{c}\text { First } \\
\text { author } \\
\text { (ref.) }\end{array}$} & \multicolumn{3}{|c|}{ Study population and setting } & \multicolumn{3}{|c|}{ Outcome measure } & \multicolumn{4}{|c|}{ Intervention description } \\
\hline & $\begin{array}{c}\text { Target } \\
\text { population }\end{array}$ & Age range $\dagger$ & $\begin{array}{l}\text { Study } \\
\text { setting }\end{array}$ & $\begin{array}{c}\text { Type of } \\
\text { cancer } \\
\text { screening }\end{array}$ & $\begin{array}{l}\text { Follow up } \\
\text { duration }\end{array}$ & $\begin{array}{c}\text { Data source for } \\
\text { screening } \\
\text { outcome }\end{array}$ & $\begin{array}{l}\text { Delivery } \\
\text { mode }\end{array}$ & $\begin{array}{l}\text { Intervention } \\
\text { components }\end{array}$ & $\begin{array}{l}\text { No. of } \\
\text { BCTS }\end{array}$ & $\begin{array}{l}\text { Theoretic } \\
\text { framework }\end{array}$ \\
\hline $\begin{array}{l}\text { Navarro } \\
(1998)\end{array}$ & Hispanic women & $\begin{array}{l}<40(68.7 \%) \\
40-49(20.2 \%)\end{array}$ & Community & $\begin{array}{l}\text { Breast, } \\
\text { Cervical, } \\
\text { BSE, CBE }\end{array}$ & $\begin{array}{l}\text { Immediately } \\
\text { post } \\
\text { intervention }\end{array}$ & SR & $\begin{array}{l}\text { Group (face to } \\
\text { face) }\end{array}$ & $\begin{array}{l}\text { Educational information, } \\
\text { practical skills training, } \\
\text { logistical assistance }\end{array}$ & 0 & SCT, SET \\
\hline $\begin{array}{l}\text { Nguyen } \\
(2009)\end{array}$ & $\begin{array}{l}\text { Asian American } \\
\text { women }\end{array}$ & $57.0(10.1)$ & $\begin{array}{l}\text { Primarily } \\
\text { home but } \\
\text { sometimes } \\
\text { community }\end{array}$ & $\begin{array}{l}\text { Breast, } \\
\text { CBE }\end{array}$ & $5-6 \mathrm{mths}$ & SR & $\begin{array}{l}\text { Group (face to } \\
\text { face) }\end{array}$ & $\begin{array}{l}\text { Flipchart, educational } \\
\text { information, educational } \\
\text { pamphlet, logistical } \\
\text { assistance }\end{array}$ & 6 & N/A \\
\hline $\begin{array}{l}\text { Nguyen } \\
(2015)\end{array}$ & $\begin{array}{l}\text { Asian American } \\
\text { men and women }\end{array}$ & $50-74$ & $\begin{array}{l}\text { Primarily } \\
\text { home but } \\
\text { sometimes } \\
\text { community }\end{array}$ & Colorectal & $6 \mathrm{mths}$ & SR & $\begin{array}{l}\text { Group (face to } \\
\text { face) }+ \\
\text { individual } \\
\text { (face to face } \\
\& \text { telephone) }\end{array}$ & $\begin{array}{l}\text { Flipchart, educational } \\
\text { information, barriers } \\
\text { counselling, logistical } \\
\text { assistance, follow up } \\
\text { reminder }\end{array}$ & 5 & N/A \\
\hline $\begin{array}{l}\text { Nguyen } \\
(2017)\end{array}$ & $\begin{array}{l}\text { Asian American } \\
\text { men and women }\end{array}$ & $62.2(6.9)$ & $\begin{array}{l}\text { Community } \\
\text { or home }\end{array}$ & Colorectal & $6 \mathrm{mths}$ & SR & $\begin{array}{l}\text { Group (face to } \\
\text { face) }+ \\
\text { individual } \\
\text { (telephone) }\end{array}$ & $\begin{array}{l}\text { Flipchart, educational } \\
\text { information, educational } \\
\text { brochure, logistical } \\
\text { assistance, follow up } \\
\text { reminder }\end{array}$ & 4 & TTM \\
\hline $\begin{array}{l}\text { Nuño } \\
(2011)\end{array}$ & Hispanic women & $59.2(7.7)$ & Home & $\begin{array}{l}\text { Breast, } \\
\text { Cervical }\end{array}$ & $12 \mathrm{mths}$ & $\mathrm{MR}+\mathrm{SR}$ & $\begin{array}{l}\text { Group (face to } \\
\text { face) }\end{array}$ & $\begin{array}{l}\text { Educational information, } \\
\text { logistical assistance }\end{array}$ & 1 & SCT \\
\hline $\begin{array}{l}\text { O’Brien } \\
(2010)\end{array}$ & Hispanic women & $32(11.0)$ & Community & Cervical & $6 \mathrm{mths}$ & $\mathrm{MR}+\mathrm{SR}$ & $\begin{array}{l}\text { Group (face to } \\
\text { face) }\end{array}$ & $\begin{array}{l}\text { Educational information, } \\
\text { educational pamphlet }\end{array}$ & 0 & HBM \\
\hline
\end{tabular}


Appendix 3. Study details and intervention summaries (Cont'd)

\begin{tabular}{|c|c|c|c|c|c|c|c|c|c|c|}
\hline \multirow[b]{2}{*}{$\begin{array}{c}\text { First } \\
\text { author } \\
\text { (ref.) }\end{array}$} & \multicolumn{3}{|c|}{ Study population and setting } & \multicolumn{3}{|c|}{ Outcome measure } & \multicolumn{4}{|c|}{ Intervention description } \\
\hline & Target population & Age range $\dagger$ & $\begin{array}{l}\text { Study } \\
\text { setting }\end{array}$ & $\begin{array}{c}\text { Type of } \\
\text { cancer } \\
\text { screening }\end{array}$ & $\begin{array}{c}\text { Follow up } \\
\text { duration }\end{array}$ & $\begin{array}{c}\text { Data source for } \\
\text { screening } \\
\text { outcome }\end{array}$ & $\begin{array}{c}\text { Delivery } \\
\text { mode }\end{array}$ & $\begin{array}{l}\text { Intervention } \\
\text { components }\end{array}$ & $\begin{array}{l}\text { No. of } \\
\text { BCTs }\end{array}$ & $\begin{array}{c}\text { Theoretic } \\
\text { framework }\end{array}$ \\
\hline $\begin{array}{l}\text { Paskett } \\
(2006)\end{array}$ & $\begin{array}{l}\text { African American, } \\
\text { Native American } \\
\text { and White women }\end{array}$ & $\begin{array}{l}55.1(54.33- \\
55.83)\end{array}$ & Home & Breast & $12-14 \mathrm{mths}$ & $\mathrm{MR}+\mathrm{SR}$ & $\begin{array}{l}\text { Individual } \\
\text { (face to face) }\end{array}$ & $\begin{array}{l}\text { Educational information, } \\
\text { educational pamphlet, } \\
\text { practical skills training, } \\
\text { barriers counselling, } \\
\text { logistical assistance, } \\
\text { follow up reminder }\end{array}$ & 5 & SLT, TTM \\
\hline $\begin{array}{l}\text { Paskett } \\
(2011)\end{array}$ & $\begin{array}{l}\text { Ohio Appalachian } \\
\text { women }\end{array}$ & 43.7 & Community & Cervical & 12 mths & $\mathrm{MR}+\mathrm{SR}$ & $\begin{array}{l}\text { Individual } \\
\text { (face to face } \\
\& \text { telephone) }\end{array}$ & $\begin{array}{l}\text { Educational information, } \\
\text { barriers counselling, } \\
\text { follow up reminder }\end{array}$ & 3 & HBM, SLT, TTM \\
\hline $\begin{array}{l}\text { Percac- } \\
\text { Lima } \\
(2009)\end{array}$ & $\begin{array}{l}\text { Predominantly } \\
\text { White and } \\
\text { Hispanic men and } \\
\text { women }\end{array}$ & $63.1(7.7)$ & Community & Colorectal & $9 \mathrm{mths}$ & MR & $\begin{array}{l}\text { Individual } \\
\text { (face to face) }\end{array}$ & $\begin{array}{l}\text { Educational information, } \\
\text { barriers counselling, } \\
\text { logistical assistance }\end{array}$ & 5 & N/A \\
\hline $\begin{array}{l}\text { Potter } \\
(2009)\end{array}$ & $\begin{array}{l}\text { Predominantly } \\
\text { White and Asian } \\
\text { men and women }\end{array}$ & $\begin{array}{l}51-64 \\
(46.9 \%) \\
65+(53.1 \%)\end{array}$ & $\begin{array}{l}\text { Telephone } \\
\text { Primary } \\
\text { eare }\end{array}$ & Colorectal & $3 \mathrm{mths}$ & MR & $\begin{array}{l}\text { Individual } \\
\text { (telephone) }\end{array}$ & $\begin{array}{l}\text { Educational information, } \\
\text { educational pamphlet, } \\
\text { barriers counselling, } \\
\text { follow up reminder }\end{array}$ & 3 & N/A \\
\hline $\begin{array}{l}\text { Russell } \\
(2010)\end{array}$ & $\begin{array}{l}\text { African American } \\
\text { women }\end{array}$ & $51.3(7.3)$ & Community & Breast & $6 \mathrm{mths}$ & $\mathrm{MR}+\mathrm{SR}$ & $\begin{array}{l}\text { Individual } \\
\text { (face to face } \\
\& \text { telephone) } \\
\text { + computer }\end{array}$ & $\begin{array}{l}\text { Computer program } \\
\text { including video } \\
\text { demonstration, barriers } \\
\text { counselling, logistical } \\
\text { assistance, follow up } \\
\text { reminder }\end{array}$ & 9 & HBM, TTM \\
\hline
\end{tabular}


Appendix 3. Study details and intervention summaries (Cont'd)

\begin{tabular}{|c|c|c|c|c|c|c|c|c|c|c|}
\hline \multirow[b]{2}{*}{$\begin{array}{c}\text { First } \\
\text { author } \\
\text { (ref.) }\end{array}$} & \multicolumn{3}{|c|}{ Study population and setting } & \multicolumn{3}{|c|}{ Outcome measure } & \multicolumn{4}{|c|}{ Intervention description } \\
\hline & Target population & Age range $\dagger$ & $\begin{array}{l}\text { Study } \\
\text { setting }\end{array}$ & $\begin{array}{c}\text { Type of } \\
\text { cancer } \\
\text { screening }\end{array}$ & $\begin{array}{c}\text { Follow up } \\
\text { duration }\end{array}$ & $\begin{array}{c}\text { Data source for } \\
\text { screening } \\
\text { outcome }\end{array}$ & $\begin{array}{l}\text { Delivery } \\
\text { mode }\end{array}$ & $\begin{array}{l}\text { Intervention } \\
\text { components }\end{array}$ & $\begin{array}{l}\text { No. of } \\
\text { BCTs }\end{array}$ & $\begin{array}{l}\text { Theoretic } \\
\text { framework }\end{array}$ \\
\hline $\begin{array}{l}\text { Sadler } \\
(2011)\end{array}$ & $\begin{array}{l}\text { African American } \\
\text { women }\end{array}$ & $40.6(13.0)$ & Community & Breast & $6 \mathrm{mths}$ & SR & $\begin{array}{l}\text { Group }+ \\
\text { individual } \\
\text { (face to face) }\end{array}$ & $\begin{array}{l}\text { Educational information, } \\
\text { media materials, practical } \\
\text { skills training }\end{array}$ & 3 & HBM \\
\hline $\begin{array}{l}\text { Sadler } \\
(2012)\end{array}$ & $\begin{array}{l}\text { Asian American } \\
\text { women }\end{array}$ & $49.1(12.0)$ & Community & $\begin{array}{l}\text { Breast, } \\
\text { CBE }\end{array}$ & $2 \mathrm{mths}$ & SR (intention) & $\begin{array}{l}\text { Individual } \\
\text { (face to face) }\end{array}$ & $\begin{array}{l}\text { Educational information, } \\
\text { educational pamphlet, } \\
\text { barriers counselling, } \\
\text { follow up reminder }\end{array}$ & 3 & N/A \\
\hline $\begin{array}{l}\text { Sung } \\
(1997)\end{array}$ & $\begin{array}{l}\text { African American } \\
\text { women }\end{array}$ & $18-97$ & Home & $\begin{array}{l}\text { Breast, } \\
\text { BSE, CBE }\end{array}$ & $6 \mathrm{mths}$ & SR & $\begin{array}{l}\text { Individual } \\
\text { (face to face) }\end{array}$ & $\begin{array}{l}\text { Educational information, } \\
\text { educational pamphlet, } \\
\text { videotape }\end{array}$ & 2 & N/A \\
\hline $\begin{array}{l}\text { Taylor } \\
(2002 a)\end{array}$ & $\begin{array}{l}\text { Asian American } \\
\text { women }\end{array}$ & $18-60+$ & Home & Cervical & $12 \mathrm{mths}$ & $\mathrm{MR}+\mathrm{SR}$ & $\begin{array}{l}\text { Group }+ \\
\text { individual } \\
\text { (face to face } \\
\text { \& telephone) }\end{array}$ & $\begin{array}{l}\text { Visual aids, barriers } \\
\text { counselling, video, } \\
\text { logistical assistance }\end{array}$ & 3 & N/A \\
\hline $\begin{array}{l}\text { Taylor } \\
(2002 b)\end{array}$ & $\begin{array}{l}\text { Asian American } \\
\text { women }\end{array}$ & $20-69$ & Home & Cervical & $6 \mathrm{mths}$ & $\mathrm{MR}+\mathrm{SR}$ & $\begin{array}{l}\text { Individual } \\
\text { (face to face } \\
\& \text { telephone) }\end{array}$ & $\begin{array}{l}\text { Educational information, } \\
\text { educational pamphlets, } \\
\text { video, follow up reminder }\end{array}$ & 9 & N/A \\
\hline $\begin{array}{l}\text { Taylor } \\
(2010)\end{array}$ & $\begin{array}{l}\text { Asian American } \\
\text { women }\end{array}$ & $\begin{array}{l}<50(45 \%) \\
\geq 50(55 \%)\end{array}$ & Home & Cervical & $6 \mathrm{mths}$ & $\mathrm{MR}+\mathrm{SR}$ & $\begin{array}{l}\text { Individual } \\
\text { (face to face } \\
\text { \& telephone) }\end{array}$ & $\begin{array}{l}\text { Educational information, } \\
\text { educational pamphlet, } \\
\text { DVD, barriers } \\
\text { counselling, follow up } \\
\text { reminder }\end{array}$ & 9 & N/A \\
\hline
\end{tabular}


Appendix 3. Study details and intervention summaries (Cont'd)

\begin{tabular}{|c|c|c|c|c|c|c|c|c|c|c|}
\hline \multirow[b]{2}{*}{$\begin{array}{l}\text { First } \\
\text { author } \\
\text { (ref.) }\end{array}$} & \multicolumn{3}{|c|}{ Study population and setting } & \multicolumn{3}{|c|}{ Outcome measure } & \multicolumn{4}{|c|}{ Intervention description } \\
\hline & Target popualtion & Age range $\dagger$ & $\begin{array}{l}\text { Study } \\
\text { setting }\end{array}$ & $\begin{array}{c}\text { Type of } \\
\text { cancer } \\
\text { screening }\end{array}$ & $\begin{array}{l}\text { Follow up } \\
\text { duration }\end{array}$ & $\begin{array}{l}\text { Data source for } \\
\text { screening } \\
\text { outcome }\end{array}$ & $\begin{array}{l}\text { Delivery } \\
\text { mode }\end{array}$ & $\begin{array}{l}\text { Intervention } \\
\text { components }\end{array}$ & $\begin{array}{l}\text { No. of } \\
\text { BCTs }\end{array}$ & $\begin{array}{l}\text { Theoretic } \\
\text { framework }\end{array}$ \\
\hline $\begin{array}{l}\text { Thompson } \\
\text { (2017) }\end{array}$ & Hispanic women & $43.9(9.8)$ & Home & Cervical & $7 \mathrm{mths}$ & MR & $\begin{array}{l}\text { Individual } \\
\text { (face to face } \\
\text { \& telephone) }\end{array}$ & $\begin{array}{l}\text { Educational information, } \\
\text { video, logistical } \\
\text { assistance, follow up } \\
\text { reminder }\end{array}$ & 8 & SCT \\
\hline $\begin{array}{l}\text { Tong } \\
(2017)\end{array}$ & $\begin{array}{l}\text { Asian American } \\
\text { men and women }\end{array}$ & $50-75$ & Community & Colorectal & $6 \mathrm{mths}$ & SR & $\begin{array}{l}\text { Group (face to } \\
\text { face) }+ \\
\text { individual } \\
\text { (telephone) }\end{array}$ & $\begin{array}{l}\text { Flipchart, educational } \\
\text { information, educational } \\
\text { pamphlet, follow up } \\
\text { reminder }\end{array}$ & 4 & SCT, TTM \\
\hline $\begin{array}{l}\text { Walsh } \\
(2010)\end{array}$ & $\begin{array}{l}\text { Hispanic \& Asian } \\
\text { American men and } \\
\text { women }\end{array}$ & $60.7(6.8)$ & $\begin{array}{l}\text { Telephone } \\
\text { Primary } \\
\text { eare }\end{array}$ & Colorectal & $9-12 \mathrm{mths}$ & SR & $\begin{array}{l}\text { Individual } \\
\text { (telephone) }\end{array}$ & $\begin{array}{l}\text { Educational information, } \\
\text { educational pamphlet, } \\
\text { barriers counselling }\end{array}$ & 3 & TTM \\
\hline Zhu (2002) & $\begin{array}{l}\text { African American } \\
\text { women }\end{array}$ & $65-85+$ & Home & $\begin{array}{l}\text { Breast, } \\
\text { BSE, CBE }\end{array}$ & 12 mths & SR & $\begin{array}{l}\text { Individual } \\
\text { (face to face) }\end{array}$ & $\begin{array}{l}\text { Educational information, } \\
\text { educational pamphlets, } \\
\text { barriers counselling }\end{array}$ & 3 & HBM, SST, TRA \\
\hline
\end{tabular}

\begin{tabular}{|c|c|c|c|c|c|c|c|c|c|c|}
\hline \multicolumn{11}{|c|}{ Cancer preventative behaviours } \\
\hline \multicolumn{4}{|c|}{ Study population and setting } & \multicolumn{3}{|c|}{ Outcome measure } & \multicolumn{4}{|c|}{ Intervention deseription } \\
\hline $\begin{array}{l}\text { First } \\
\text { (ref.) }\end{array}$ & Target population & Age rangef & $\begin{array}{l}\text { Study } \\
\text { setting }\end{array}$ & $\begin{array}{l}\text { Type-of } \\
\text { eancer }\end{array}$ & $\begin{array}{l}\text { Follow } \\
\text { duration }\end{array}$ & $\begin{array}{l}\text { Outcome } \\
\text { measure }\end{array}$ & $\begin{array}{l}\text { Delivery } \\
\text { mode }\end{array}$ & $\begin{array}{l}\text { Intervention } \\
\text { components }\end{array}$ & $\begin{array}{l}\text { No.of } \\
\text { BCTs }\end{array}$ & $\begin{array}{l}\text { Theoretie } \\
\text { framework }\end{array}$ \\
\hline
\end{tabular}




\section{Page 89 of 95}

Psycho-Oncology

\begin{tabular}{|c|c|c|c|c|c|c|c|c|c|c|}
\hline $\begin{array}{l}\text { Calmpbell } \\
\text { (2004) }\end{array}$ & $\begin{array}{l}\text { African American } \\
\text { men and women }\end{array}$ & $\begin{array}{l}<40(25 \%) \\
40-49 \\
(27.5 \%) \\
50+(47.5 \%)\end{array}$ & $\begin{array}{l}\text { Communit } \\
\text { y }\end{array}$ & $\begin{array}{l}\text { Colorecta } \\
1\end{array}$ & 12 mths & $\begin{array}{l}\text { Fruit, } \\
\text { vegetable and } \\
\text { fat } \\
\text { consumption, } \\
\text { physical } \\
\text { activity§ }\end{array}$ & $\begin{array}{l}\text { Group (face } \\
\text { to face) }\end{array}$ & $\begin{array}{l}\text { Educational } \\
\text { information, organizing } \\
\text { exercise groups }\end{array}$ & 2 & $\begin{array}{l}\text { SCT, TTM, HBM, } \\
\text { SST }\end{array}$ \\
\hline $\begin{array}{l}\text { Halvas } \\
\text { (1998) }\end{array}$ & $\begin{array}{l}\text { Afriean American } \\
\text { and White women }\end{array}$ & 18-30+ & $\begin{array}{l}\text { Communit } \\
y\end{array}$ & Any & 8 mths & $\begin{array}{l}\text { Fruit andveg } \\
\text { consumption }\end{array}$ & $\begin{array}{l}\text { Group }= \\
\text { individual } \\
\text { (face to } \\
\text { face) }\end{array}$ & $\begin{array}{l}\text { Educational } \\
\text { information, educational } \\
\text { pamphlets, cooking } \\
\text { demonstrations, video }\end{array}$ & 6 & ТТМ \\
\hline
\end{tabular}

Note: †Mean and SD or CI reported if range not reported. If mean not reported, age categories are given with corresponding percentages

‡No cancer screening outcome measured; colorectal cancer knowledge and intent to complete colorectal cancer screening only

§outeome measure for colorectal sereening not extracted because of healtheare professional involvement in activities related to sereening

BSE, breast self-examination; CBE, clinical breast examination; MR, medical records; SR, self-report; BCTs, behaviour change techniques SCT, Social Cognitive Theory;

HBM, Health Belief Model; TTM, Transtheoretical Model; TRA, Theory of Reasoned Action; SET, Self-Efficacy Theory; SLT, Social Learning Theory; SST, Social

Support Theory; N/A, no theory mentioned 
Appendix 4. Risk of bias graph

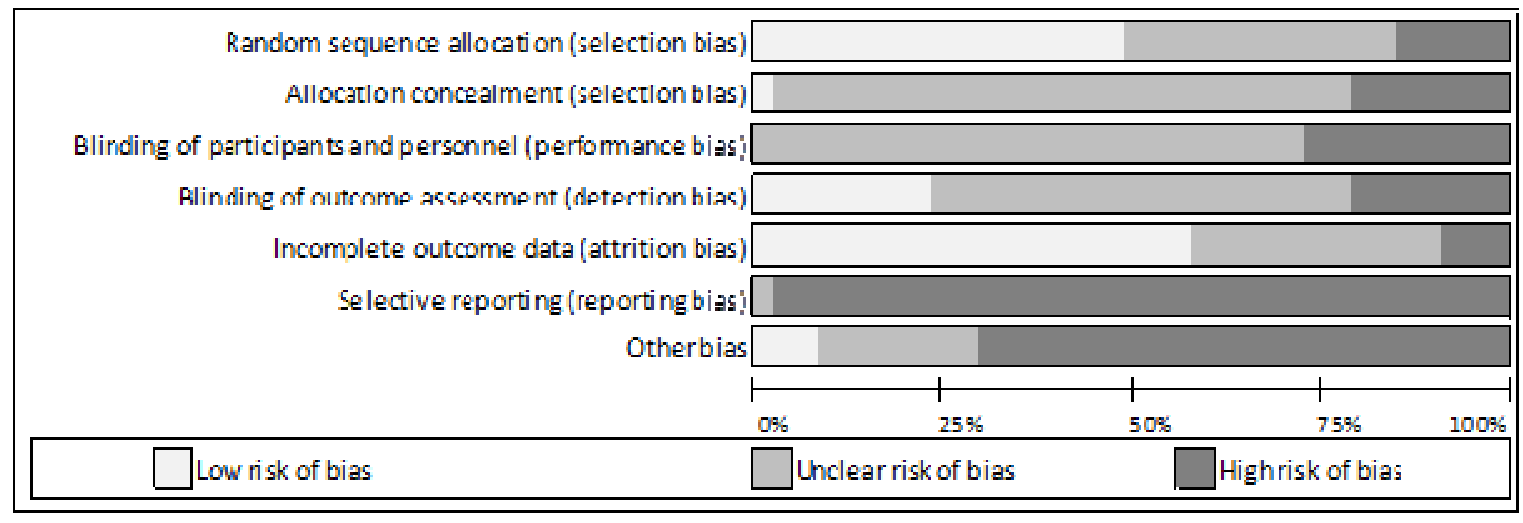

1

2

3

4

5

6

7

10

11

12

13

14

15

16

17

18

19

20

21

22

23

24

25

26

27

28

29

30

31

32

33

34

35

36

37

38

39

40

41

42

43

44

45

46

47

48

49

50

51

52

53

54

55

56

57

58

59

60 
Appendix 5. Meta-analysis of cancer screening uptake in non-adherent samples receiving $\mathbf{L}$ CBHW interventions, in comparison to control conditions. Box size represents study weighting. Diamond represents overall effect size and 95\% confidence intervals

Study name

Byrd et al. 2013

Duan et al. 2000

Fernandez et al. 2009

Goelen et al. 2010

Han et al. 2017

Jandorf et al. 2008

Lam et al. 2003

Mock et al. 2007

Nguyen et al. 2015

Nuno et al. 2011

O'Brien et al. 2010

Paskett et al. 2006

Percac-Lima et al. 2009

Potter et al. 2009

Russell et al. 2010

Sadler et al. 2011

Sung et al. 1997

Taylor et al. 2002b

Taylor et al. 2010

Thompson et al. 2017

\section{Statistics for each study}

$\begin{array}{rrrrrr}\begin{array}{c}\text { Odds } \\ \text { ratio }\end{array} & \begin{array}{c}\text { Lower } \\ \text { limit }\end{array} & \begin{array}{c}\text { Upper } \\ \text { limit }\end{array} & \text { Z-Value } & \text { p-Value } \\ 2.619 & 1.611 & 4.257 & 3.882 & 0.000 \\ 1.119 & 0.693 & 1.807 & 0.461 & 0.645 \\ 1.348 & 0.789 & 2.302 & 1.093 & 0.274 \\ 1.285 & 1.097 & 1.505 & 3.110 & 0.002 \\ 11.660 & 7.349 & 18.502 & 10.428 & 0.000 \\ 1.890 & 1.286 & 2.777 & 3.239 & 0.001 \\ 2.807 & 1.305 & 6.036 & 2.641 & 0.008 \\ 2.292 & 1.413 & 3.717 & 3.360 & 0.001 \\ 5.426 & 3.785 & 7.777 & 9.207 & 0.000 \\ 2.492 & 1.384 & 4.488 & 3.042 & 0.002 \\ 8.680 & 1.879 & 40.090 & 2.768 & 0.006 \\ 1.968 & 1.477 & 2.624 & 4.618 & 0.000 \\ 2.794 & 2.062 & 3.786 & 6.631 & 0.000 \\ 1.505 & 1.214 & 1.867 & 3.725 & 0.000 \\ 4.730 & 2.393 & 9.350 & 4.469 & 0.000 \\ 1.988 & 1.159 & 3.407 & 2.498 & 0.012 \\ 1.366 & 0.662 & 2.817 & 0.844 & 0.398 \\ 2.636 & 1.509 & 4.605 & 3.405 & 0.001 \\ 2.133 & 0.977 & 4.653 & 1.902 & 0.057 \\ 2.012 & 1.262 & 3.209 & 2.935 & 0.003 \\ 2.401 & 1.854 & 3.110 & 6.638 & 0.000\end{array}$

\section{Odds ratio and $95 \% \mathrm{Cl}$}

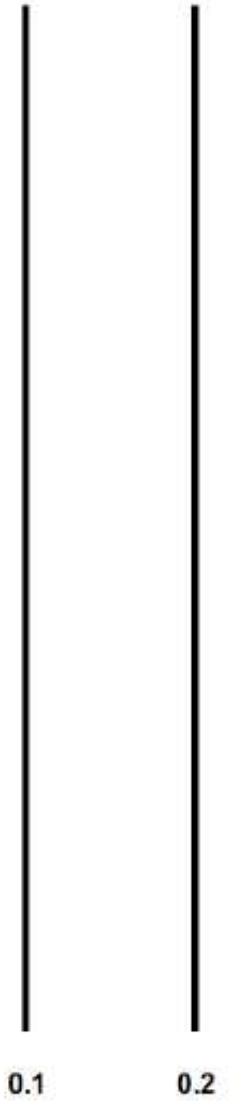

0.1
Favours Control

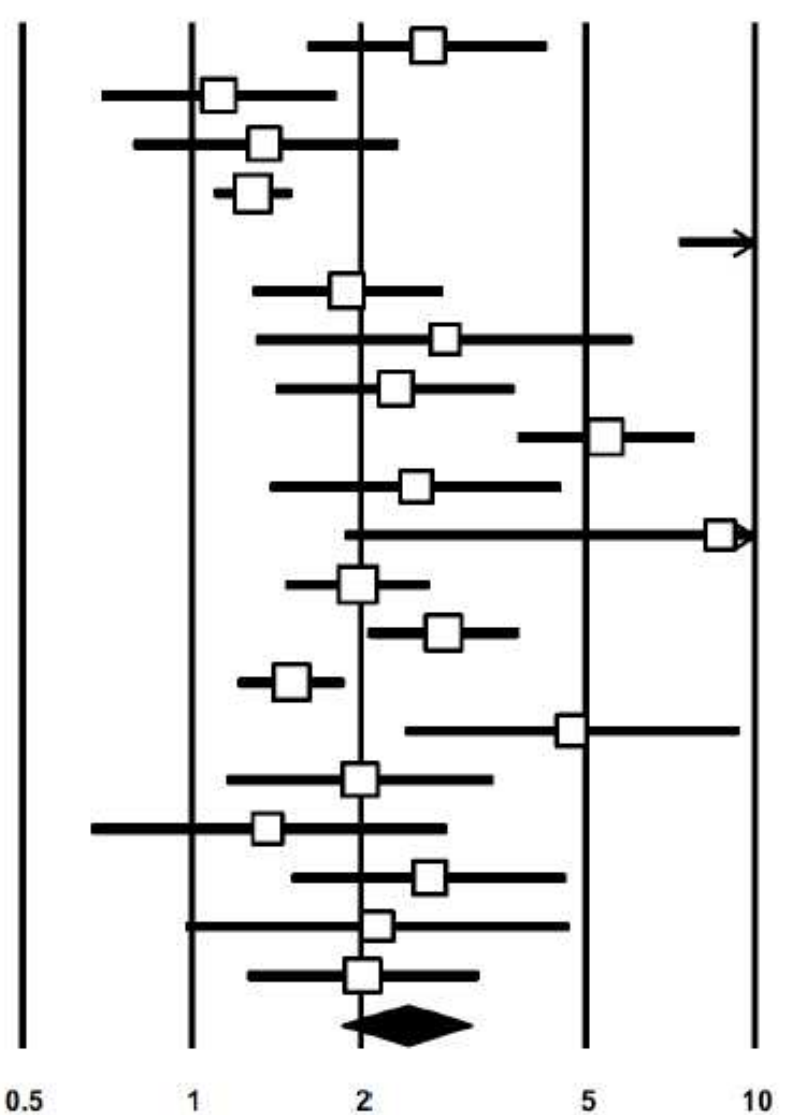

Favours Intervention 
Appendix 6. Mixed-effects meta-regression of number of BCTs

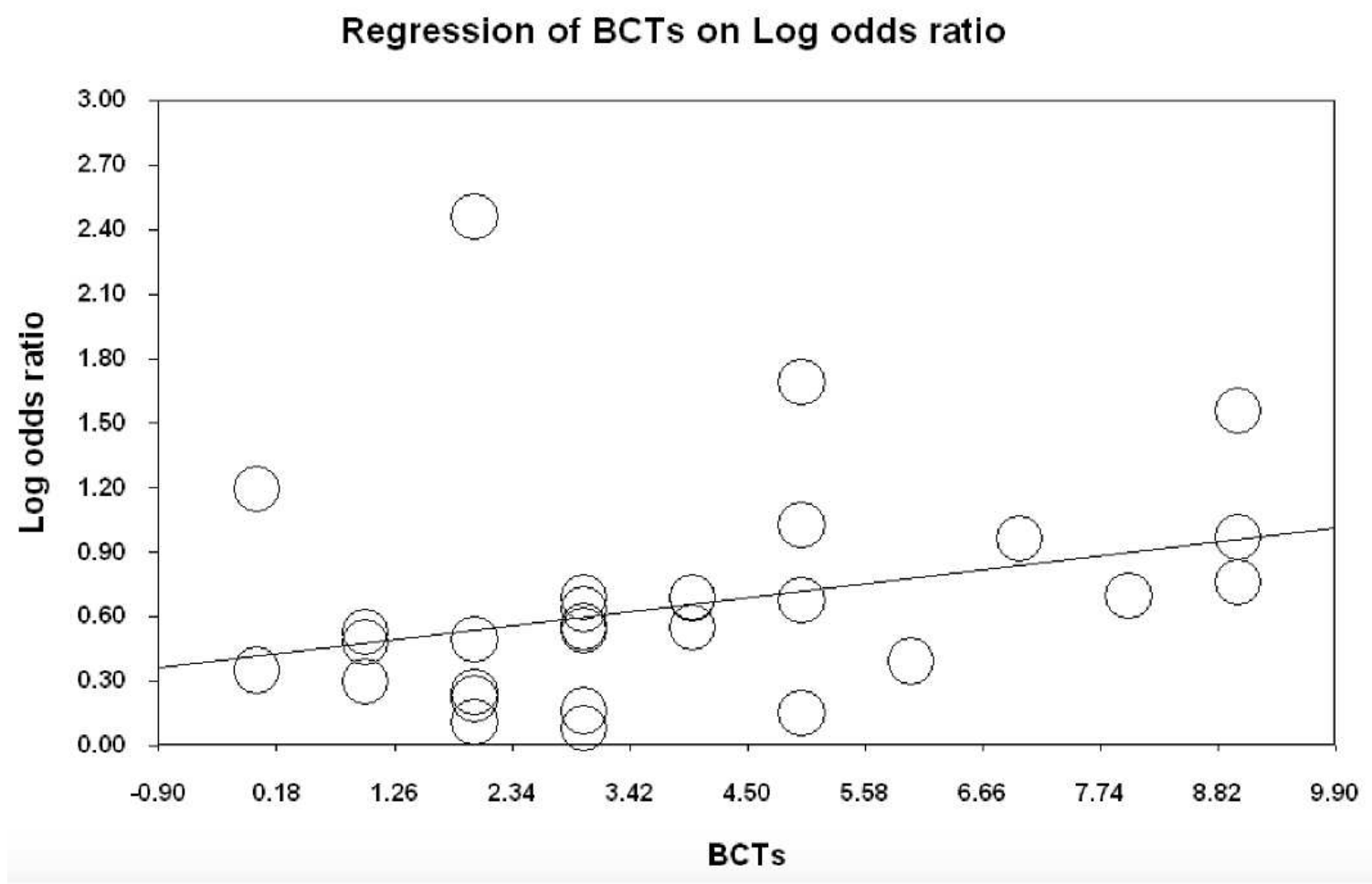

http://mc.manuscriptcentral.com/pon 


\begin{tabular}{l} 
Group by \\
High or Low \\
3 or less \\
3 or less \\
3 or less \\
3 or less \\
3 or less \\
3 or less \\
3 or less \\
3 or less \\
3 or less \\
3 or less \\
3 or less \\
3 or less \\
3 or less \\
3 or less \\
3 or less \\
3 or less \\
3 or less \\
3 or less \\
4 or more \\
\hline 4 or more \\
\hline 4 or more \\
4 or more \\
4 or more \\
4 or more \\
4 or more \\
4 or more \\
4 or more \\
4 or more \\
4 or more \\
4 or more \\
4 or more \\
4 or more \\
\hline
\end{tabular}

Study name

Duan et al. 2000

Femandez et al. 2009 Goelen et al. 2010

Han et al. 2017

Jandorf et al. 2008

Lam et al. 2003

Mock et al. 2007

Navarto et al. 1998
Nuno et al. 2011

O'Brien et al. 2010

Paskett et al. 2011

Potter et al. 2009

Sadler et al. 2011

Sung et al. 1997

Tayor et al. 2002a

Zhu et al. 2002

Byrd et al. 2013 Hunter et al. 2004

Jo et al. 2017

Nguyen et al. 2017

Nguygen et at. 2009

Nguygen et a.. 2015

Percac-Lima et al. 2009

Russel et al. 2010

Taylor et al. 2002b

Taylor et al. 2010

Thompson et al. 2017
Tong et al. 2017
Statistics for each study Odds Lower Upper

1619 -Value $\mathrm{p}$-Value Intervention Control

$\begin{array}{llllll}1.619 & 1.044 & 2.511 & 2.151 & 0.031 & 264\end{array}$

$\begin{array}{lllll}1.348 & 0.789 & 2.302 & 1.093 & 0.274 \\ 1.285 & 1.097 & 1.505 & 3.110 & 0.002\end{array}$

$\begin{array}{rrrrr}11.660 & 7.349 & 18.502 & 10.428 & 0.000\end{array}$

$\begin{array}{llllll}1.890 & 1.286 & 2.777 & 3.239 & 0.001\end{array}$

$\begin{array}{llllll}1.244 & 0.778 & 1.989 & 0.911 & 0.362\end{array}$

$\begin{array}{lllll}1.637 & 1.250 & 2.143 & 3.586 & 0.000\end{array}$

$\begin{array}{lllll}1.419 & 0.928 & 2.169 & 1.617 & 0.106\end{array}$

$\begin{array}{llllll}1.697 & 1.104 & 2.607 & 2.411 & 0.016\end{array}$

$\begin{array}{llllll}3.302 & 1.240 & 8.794 & 2.390 & 0.017\end{array}$

$\begin{array}{lllll}1.741 & 1.043 & 2.906 & 2.119 & 0.034\end{array}$

$\begin{array}{lllll}1.176 & 1.046 & 1.322 & 2.721 & 0.007\end{array}$

$\begin{array}{lllll}1.988 & 1.159 & 3.407 & 2.498 & 0.012 \\ 1.116 & 0.713 & 1.746 & 0.481 & 0.631\end{array}$

$\begin{array}{lllll}1.116 & 0.713 & 1.746 & 0.481 & 0.631\end{array}$

$\begin{array}{llllll}1.084 & 0.626 & 1.877 & 0.288 & 0.773\end{array}$

$\begin{array}{llllll}1.084 & 0.700 & 1.678 & 0.361 & 0.718\end{array}$

$\begin{array}{lllll}1.660 & 1.342 & 2.055 & 4.665 & 0.000\end{array}$

$\begin{array}{llllll}2.619 & 1.611 & 4.257 & 3.882 & 0.000\end{array}$

$\begin{array}{lllll}1.986 & 0.893 & 4.414 & 1.683 & 0.092\end{array}$

$\begin{array}{llllll}1.165 & 0.759 & 1.786 & 0.698 & 0.485\end{array}$

$\begin{array}{llllll}1.997 & 1.436 & 2.778 & 4.111 & 0.000\end{array}$

$\begin{array}{lllll}1.480 & 1.104 & 1.986 & 2.617 & 0.009\end{array}$

$\begin{array}{lllll}5.426 & 3.785 & 7.777 & 9.207 & 0.000\end{array}$

$\begin{array}{llllll}1.968 & 1.477 & 2.624 & 4.618 & 0.000\end{array}$

$\begin{array}{lllll}2.794 & 2.062 & 3.786 & 6.631 & 0.000 \\ 4.730 & 2393 & 9.350 & 4.469 & 0.000\end{array}$

$\begin{array}{llllll}2.636 & 1.509 & 4.605 & 3.405 & 0.001\end{array}$

$\begin{array}{lllll}2.133 & 0.977 & 4.653 & 1.902 & 0.057\end{array}$

$\begin{array}{lllll}2.012 & 1.262 & 3.209 & 2.935 & 0.003\end{array}$

$\begin{array}{lllll}1.729 & 1.117 & 2.675 & 2.459 & 0.014\end{array}$
Sample size

\begin{tabular}{cc}
264 & 258 \\
170 & 184 \\
1940 & 1940 \\
278 & 282 \\
308 & 179 \\
182 & 191 \\
453 & 458 \\
199 & 162 \\
183 & 188 \\
34 & 36 \\
127 & 125 \\
3144 & 2080 \\
112 & 120 \\
163 & 158 \\
113 & 115 \\
571 & 394 \\
162 & 162 \\
8403 & 7030 \\
153 & 153 \\
50 & 51 \\
177 & 161 \\
360 & 360 \\
543 & 546 \\
316 & 311 \\
433 & 418 \\
409 & 814 \\
89 & 90 \\
129 & 137 \\
118 & 116 \\
146 & 149 \\
161 & 168 \\
3084 & 3473 \\
\hline
\end{tabular}

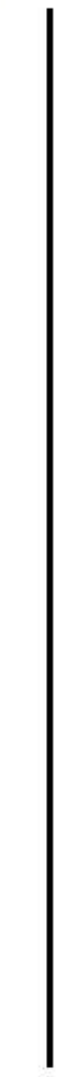

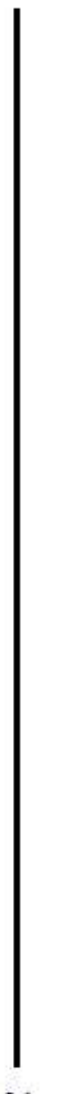

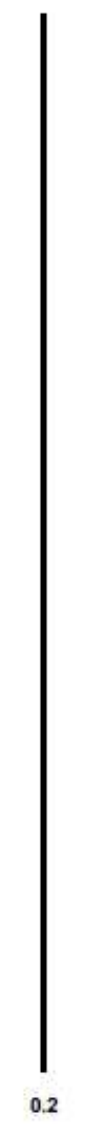

Odds ratio and $95 \% \mathrm{CI}$

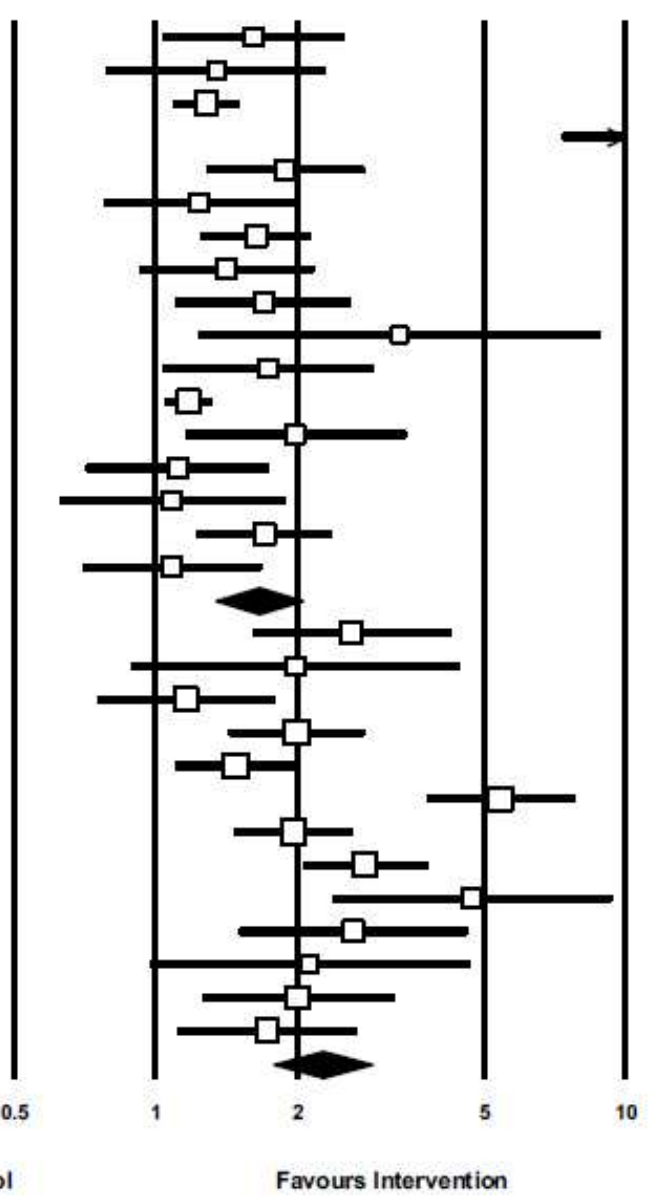

Favours Intervention 
Records identified through database searching

First search: ( $n=1885$; 807 Embase, 559 Medline, 238

Cinahl, 211 PsycINFO, 61 BNI \& 9 AMED)

Second search: ( $n=579$; 307 Embase, 143 Medline, 83

Cinahl, 33 PsycINFO \& 13 BNI)

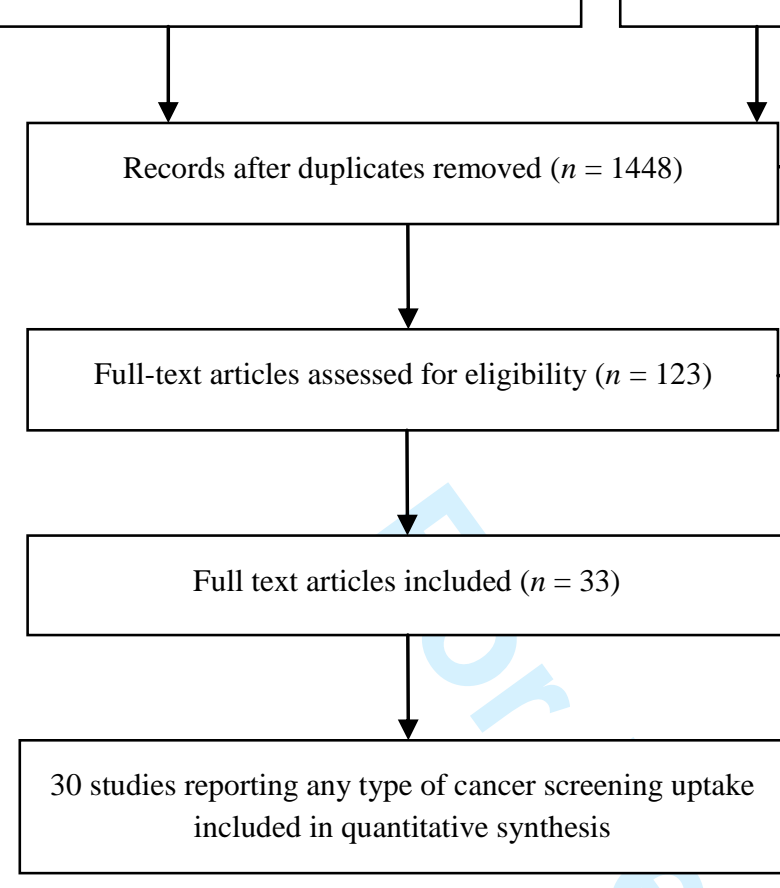

Additional records identified through other sources

$$
(n=29)
$$


Study name

Byrd et al. 2013

Duan et al. 2000

Fernandez et al. 2009

Goelen et al. 2010

Han et al. 2017

Hunter et al. 2004

Jandorf et al. 2008

Jo et al. 2017

Lam et al. 2003

Mock et al. 2007

Navarro et al. 1998

Nguyen et al. 2009

Nguyen et al. 2015

Nguyen et al. 2017

Nuno et al. 2011

O'Brien et al. 2010

Paskett et al. 2006

Paskett et al. 2011

Percac-Lima et al. 2009

Potter et al. 2009

Russell et al. 2010

Sadler et al. 2011

Sung et al. 1997

Taylor et al. 2002a

Taylor et al. 2002b

Taylor et al. 2010

Thompson et al. 2017

Tong et al. 2017

Walsh et al. 2010

Zhu et al. 2002
Statistics for each study

\section{Odds Lower Upper}

ratio limit limit Z-Value $p$-Value Intervention Control

$\begin{array}{lllll}2.619 & 1.611 & 4.257 & 3.882 & 0.000\end{array}$

$\begin{array}{lllll}1.619 & 1.044 & 2.511 & 2.151 & 0.031\end{array}$

$\begin{array}{llllll}1.348 & 0.789 & 2.302 & 1.093 & 0.274\end{array}$

$\begin{array}{lllll}1.285 & 1.097 & 1.505 & 3.110 & 0.002\end{array}$

$\begin{array}{lllll}11.660 & 7.349 & 18.502 & 10.428 & 0.000\end{array}$

$\begin{array}{lllll}1.986 & 0.893 & 4.414 & 1.683 & 0.092\end{array}$

$\begin{array}{llllll}1.890 & 1.286 & 2.777 & 3.239 & 0.001\end{array}$

$\begin{array}{lllll}1.165 & 0.759 & 1.786 & 0.698 & 0.485\end{array}$

$\begin{array}{lllll}1.244 & 0.778 & 1.989 & 0.911 & 0.362\end{array}$

$\begin{array}{lllll}1.637 & 1.250 & 2.143 & 3.586 & 0.000\end{array}$

$\begin{array}{lllll}1.419 & 0.928 & 2.169 & 1.617 & 0.106\end{array}$

$\begin{array}{llllll}1.480 & 1.104 & 1.986 & 2.617 & 0.009\end{array}$

$\begin{array}{lllll}5.426 & 3.785 & 7.777 & 9.207 & 0.000\end{array}$

$\begin{array}{llllll}1.997 & 1.436 & 2.778 & 4.111 & 0.000\end{array}$

$\begin{array}{lllll}1.697 & 1.104 & 2.607 & 2.411 & 0.016\end{array}$

$\begin{array}{llllll}3.302 & 1.240 & 8.794 & 2.390 & 0.017\end{array}$

$\begin{array}{lllll}1.968 & 1.477 & 2.624 & 4.618 & 0.000\end{array}$

$\begin{array}{llllll}1.741 & 1.043 & 2.906 & 2.119 & 0.034\end{array}$

$\begin{array}{lllll}2.794 & 2.062 & 3.786 & 6.631 & 0.000\end{array}$

$\begin{array}{lllll}1.176 & 1.046 & 1.322 & 2.721 & 0.007\end{array}$

$\begin{array}{lllll}4.730 & 2.393 & 9.350 & 4.469 & 0.000\end{array}$

$\begin{array}{llllll}1.988 & 1.159 & 3.407 & 2.498 & 0.012\end{array}$

$\begin{array}{lllll}1.116 & 0.713 & 1.746 & 0.481 & 0.631\end{array}$

$\begin{array}{llllll}1.084 & 0.626 & 1.877 & 0.288 & 0.773\end{array}$

$\begin{array}{llllll}2.636 & 1.509 & 4.605 & 3.405 & 0.001\end{array}$

$\begin{array}{lllll}2.133 & 0.977 & 4.653 & 1.902 & 0.057\end{array}$

$\begin{array}{lllll}2.012 & 1.262 & 3.209 & 2.935 & 0.003\end{array}$

$\begin{array}{lllll}1.729 & 1.117 & 2.675 & 2.459 & 0.014\end{array}$

$\begin{array}{lllll}1.709 & 1.229 & 2.376 & 3.185 & 0.001\end{array}$

$\begin{array}{lllll}1.084 & 0.700 & 1.678 & 0.361 & 0.718\end{array}$

$\begin{array}{lllll}1.901 & 1.597 & 2.263 & 7.220 & 0.000\end{array}$
Sample size

\begin{tabular}{cc}
153 & 153 \\
264 & 258 \\
170 & 184 \\
1940 & 1940 \\
278 & 282 \\
50 & 51 \\
308 & 179 \\
177 & 161 \\
182 & 191 \\
453 & 458 \\
199 & 162 \\
543 & 546 \\
316 & 311 \\
360 & 360 \\
183 & 188 \\
34 & 36 \\
433 & 418 \\
127 & 125 \\
409 & 814 \\
3144 & 2080 \\
89 & 90 \\
112 & 120 \\
163 & 158 \\
113 & 115 \\
129 & 137 \\
118 & 116 \\
146 & 149 \\
161 & 168 \\
571 & 394 \\
162 & 162 \\
11487 & 10503 \\
& \\
\hline 13
\end{tabular}

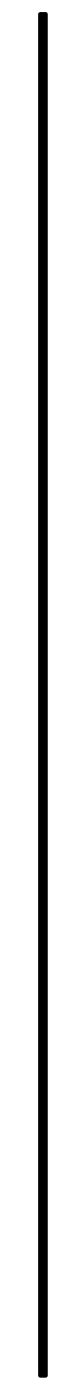

0.1

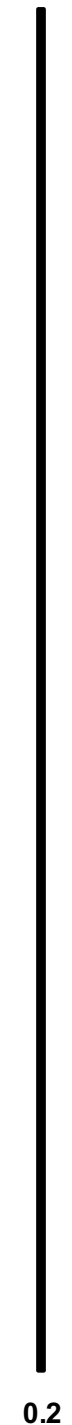

Favours Control
Odds ratio and $95 \% \mathrm{Cl}$

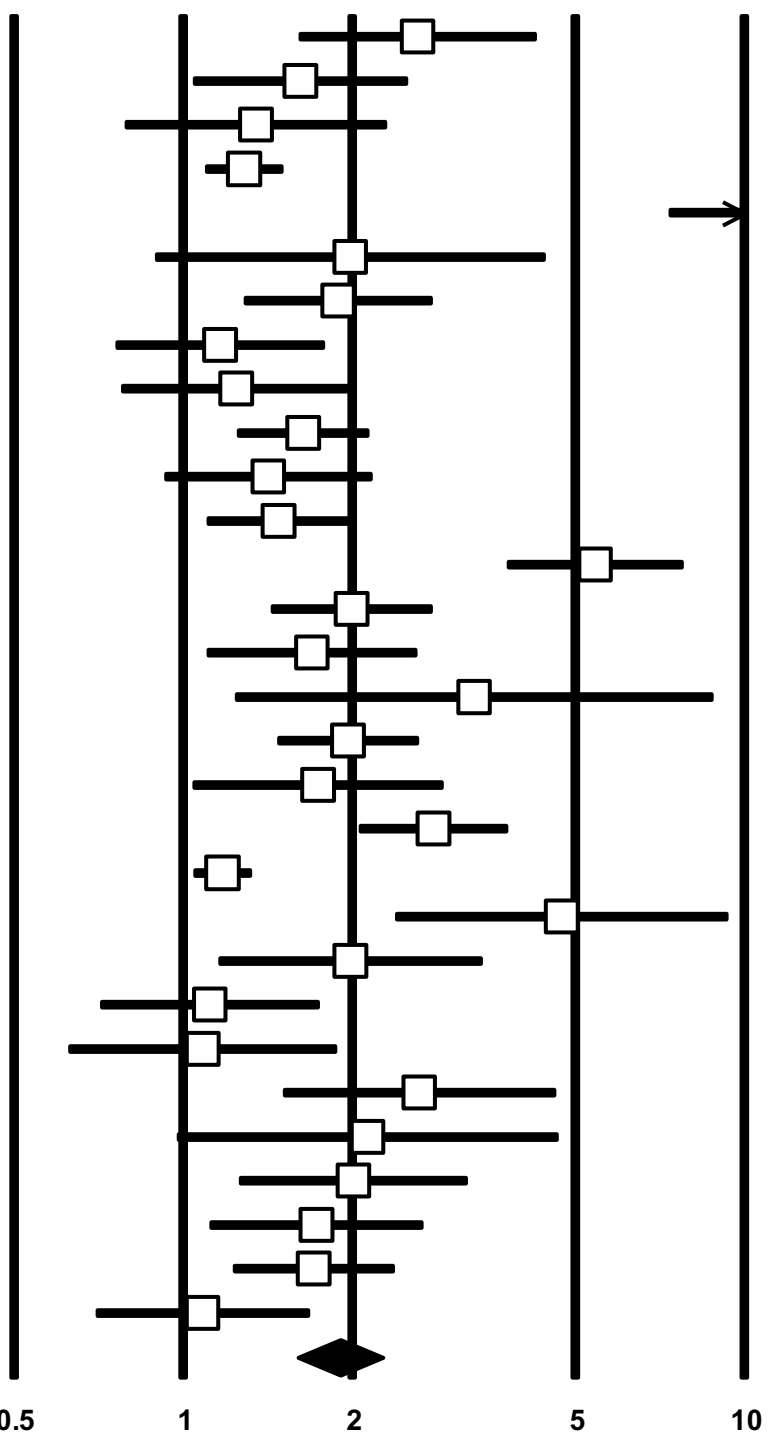

Favours Intervention 